UNIVERSIDADE DE SÃO PAULO

FACULDADE DE ZOOTECNIA E ENGENHARIA DE ALIMENTOS

VALQUIRIA APARECIDA FORTUNATO

Modelagem computacional de biorreatores de fluxo contínuo para tratamento e aproveitamento de efluentes agroindustriais 


\section{Modelagem computacional de biorreatores de fluxo contínuo para tratamento e aproveitamento de efluentes agroindustriais}

(Versão corrigida)

Dissertação apresentada à Faculdade de Zootecnia e Engenharia de Alimentos da Universidade de São Paulo, como parte dos requisitos para obtenção do título de mestre em Ciências do programa de pós-graduação em Engenharia e Ciência dos Materiais.

Área de Concentração: Desenvolvimento, Caracterização e Aplicação de Materiais Voltados à Agroindústria.

Orientador: Prof. Dr. José Antonio Rabi. 
Ficha catalográfica elaborada pelo Serviço de Biblioteca e Informação, FZEA/USP, com os dados fornecidos pelo(a) autor(a)

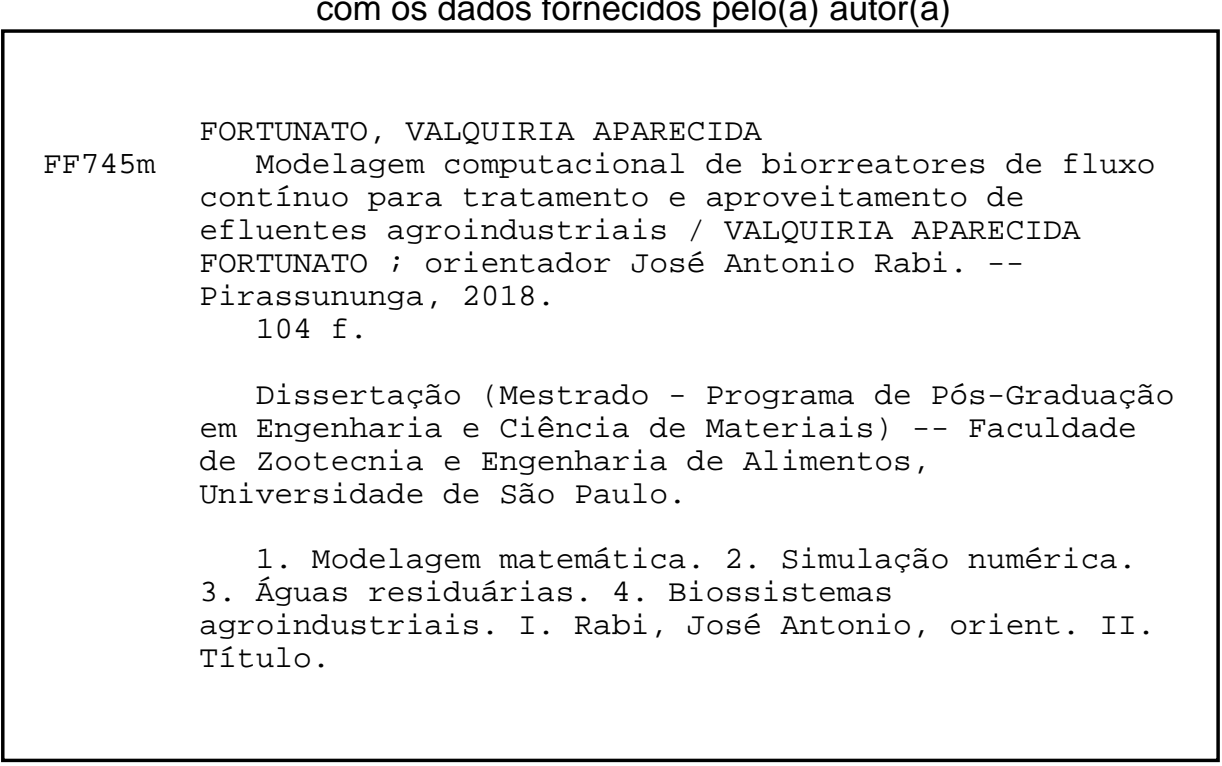

Permitida a cópia total ou parcial deste documento, desde que citada a fonte - o autor 


\section{DEDICATÓRIA}

A Deus, minha família e meu noivo. 


\section{AGRADECIMENTOS}

A Deus por estar sempre presente em tudo em minha vida, por me guiar e me iluminar em todos os momentos.

Ao meu noivo Alexandre pela paciência, pelo amor, pelo apoio e por me inspirar e me fortalecer nos momentos que mais precisei, ao longo da caminhada do meu mestrado. Ao meu pai Sebastião (sempre presente no meu coração), minha mãe Elizabeth e meus irmãos Leandro e Thiago, que sempre me apoiaram e me ajudaram nos estudos.

Ao Tony, meu fiel companheiro de quatro patas.

Aos meus padrinhos Marli e Marquinho, aos meus sobrinhos, avós, meus sogros Fátima e

Paulo e aos meus queridos amigos, que também tiveram papel importante nesta etapa.

Ao meu orientador, professor Dr. José Antonio Rabi por ter aceitado me orientar com a limitação de horário devido ao meu trabalho e por toda ajuda, paciência e disponibilidade em me auxiliar à distância e em horários flexíveis.

Ao professor Rogers Ribeiro por sua disponibilidade em me ajudar, sempre que necessário. Por fim, à Universidade de São Paulo pela oportunidade de realizar o meu mestrado junto à Faculdade de Zootecnia e Engenharia de Alimentos e à empresa Agroceres pela oportunidade de conciliar meu trabalho e mestrado. 


\section{RESUMO}

FORTUNATO, V. A. Modelagem computacional de biorreatores de fluxo contínuo para tratamento e aproveitamento de efluentes agroindustriais. 2018. Dissertação (Mestrado) Faculdade de Zootecnia e Engenharia de Alimentos, Universidade de São Paulo, Pirassununga, 2018.

O tratamento de efluentes por digestão anaeróbia tem sido amplamente modelado via ADM1 (Anaerobic Digestion Model No. 1) desenvolvido pela IWA (International Water Association). Tal modelo é dinâmico de modo que concentrações das espécies químicas no interior do reator variam com o tempo, sendo matematicamente regidas por equações diferenciais ordinárias ou algébricas conforme a respectiva cinética química. Este trabalho teve como objetivo adaptar o modelo ADM1 para tratamento e posterior aproveitamento de efluentes agroindustriais, com interesse futuro ao tratamento anaeróbio da vinhaça de cana de açúcar. O presente trabalho considerou biorreatores contínuos de mistura perfeita (CSTR Continuously Stirred Tank Reactor) e a solução numérica das equações governantes foi programada em linguagem Python. O modelo computacional implementado se mostrou aplicável e pode ser utilizado em demais pesquisas que se baseiam no modelo ADM1 de digestão anaeróbia para tratamento de efluentes agroindustriais, considerando possíveis adaptações devido à especificidade de cada tipo de efluente.

Palavras-chave: Modelagem matemática. Simulação numérica. Águas residuárias. Biossistemas agroindustriais. 


\begin{abstract}
FORTUNATO, V. A. Computational modelling of continuous flow reactors for agroindustrial effluent treatment and exploitation. 2018. Dissertation (MSc) - Faculdade de Zootecnia e Engenharia de Alimentos, Universidade de São Paulo, Pirassununga, 2018.
\end{abstract}

The wastewater treatment by anaerobic digestion has been extensively modelled by ADM1 (Anaerobic Digestion Model No. 1) developed by IWA (International Water Association). This model is dynamic so that chemical species concentrations within the reactor vary over time, being mathematically governed by either ordinary differential equations or algebraic equations according to their chemical kinetics. This research aimed at adapting the ADM1 model for treatment and subsequent use of agroindustrial effluents, with future interest in the anaerobic treatment of sugarcane vinasse. The present research considered Continuously Stirred Tank Reactor (CSTR) and the numerical solution of the governing equations was programmed in Python language. The computational model implemented was applicable and can be used in other studies that are based on the ADM1 model of anaerobic digestion for the treatment of agroindustrial effluents, considering possible adaptations due to the specificity of each type of effluent.

Keywords: Mathematical modelling. Numerical simulation. Wastewater. Agroindustrial biosystems. 


\section{LISTA DE ILUSTRAÇÕES}

Figura 1 - Esquema dos componentes e processos da digestão anaeróbia baseada no modelo ADM1

Figura 2 - Algoritmo de programação do modelo ADM1

Figura 3 - Esquema ilustrativo das concentrações e nomenclaturas definidas no reator 42

Figura 4 - Perfis de concentração comparativos da simulação de Fortunato et al. (2018) em 1 D, na saída do reator versus resultados das simulações iniciais deste. 52

Figura 5 - Perfil de concentração das espécies solúveis: açúcares (a), valerato (b), butirato (c), propionato (d) acetato (e) e hidrogênio (f), metano (g) e carbono inorgânico (h) 55

Figura 6 - Perfil de concentração das espécies: nitrogênio inorgânico solúvel (a), biomassa de açúcares (b), biomassa de butirato e valerato (c) e biomassa de propionato (d), biomassa de acetato (e), biomassa de hidrogênio (f), cátions (g), ânions (h)

Figura 7 - Perfil de concentração das espécies solúveis: ácido valérico (a), ácido butírico (b), ácido propiônico (c), ácido acético (d), bicarbonato (e), amônia (f), gás hidrogênio (g) e gás metano (h)

Figura 8 - Perfil de concentração das espécies: gás $\mathrm{CO}_{2}$ (a), cálcio (b), inerte solúvel (c), inerte particulado (d), lactato solúvel (e), lactato do processo de fermentação (f), lactato do processo de oxidação (g), DQO total (h)

Figura 9 - pH calculado pela Simulação 1

Figura 10 - Perfil de concentração das espécies solúveis: açúcares (a), valerato (b), butirato (c), propionato (d) acetato (e) e hidrogênio (f), metano (g) e carbono inorgânico (h) 61

Figura 11 - Perfil de concentração das espécies: bicarbonato (a), amônia (b), gás hidrogênio

Figura 12 - pH calculado pela Simulação 1

Figura 13 - Perfil de concentração das espécies solúveis: açúcares (a), valerato (b), butirato (c), propionato (d) acetato (e) e hidrogênio (f), metano (g) e carbono inorgânico (h) 64 
Figura 14 - Perfil de concentração das espécies: nitrogênio inorgânico solúvel (a), biomassa de açúcares (b), biomassa de butirato e valerato (c) e biomassa de propionato (d), biomassa de acetato (e), biomassa de hidrogênio (f), cátions (g), ânions (h)

Figura 15 - Perfil de concentração das espécies solúveis: ácido valérico(a), ácido butírico (b), ácido propiônico (c), ácido acético (d), bicarbonato (e), amônia (f), gás $\mathrm{H}_{2}$ (g) e gás $\mathrm{CH}_{4}$ (h)66

Figura 16 - Perfil de concentração das espécies: gás $\mathrm{CO}_{2}(a)$, cálcio (b), inerte solúvel (c), inerte particulado (d), lactato solúvel (e), lactato do processo de fermentação (f), lactato do processo de oxidação (g), DQO total (h)

Figura 17 - pH calculado pela Simulação 1 68

Figura 18 - Perfil de concentração das espécies solúveis: açúcares (a), valerato (b), butirato (c), propionato (d) acetato (e) e hidrogênio (f), metano (g) e carbono inorgânico (h)

Figura 19 - Perfil de concentração das espécies: nitrogênio inorgânico solúvel (a), biomassa de açúcares (b), biomassa de butirato e valerato (c) e biomassa de propionato (d), biomassa de acetato (e), biomassa de hidrogênio (f), cátions (g), ânions (h) .

Figura 20 - Perfil de concentração das espécies solúveis: ácido valérico(a), ácido butírico (b), ácido propiônico (c), ácido acético (d), bicarbonato (e), amônia (f), gás hidrogênio (g) e gás metano (h)

Figura 21 - Perfil de concentração das espécies: gás $\mathrm{CO}_{2}(\mathrm{a})$, cálcio (b), inerte solúvel (c), inerte particulado (d), lactato solúvel (e), lactato do processo de fermentação (f), lactato do processo de oxidação (g), DQO total (h).

Figura 22 - pH calculado pela Simulação 2-A

Figura 23 - Comparação de diferentes números de iterações $(n)$ para as concentrações das espécies solúveis: açúcares, valerato, butirato, propionato e acetato, na simulação 2

Figura 24 - Perfil de concentração das espécies solúveis: açúcares (a), aminoácidos (b), ácidos graxos (c), valerato (d), butirato (e), propionato (f), acetato (g) e metano (h) 89

Figura 25 - Perfil de concentração das espécies: carbono inorgânico (a), nitrogênio inorgânico (b), compósitos (c) e biomassa de: carboidratos (d), proteínas (e), lipídeos (f), açúcares (g) e aminoácidos (h) . 
Figura 26 - Perfil de concentração das biomassa de: ácidos graxos (a), butirato e valerato (b), propionato (c) acetato (d), hidrogênio (e) e cátions (f), ânions (g) e ácido valérico (h) solúveis

Figura 27 - Perfil de concentração das espécies: ácido butírico (a), ácido propiônico (b), ácido acético (c), bicarbonato (d), $\mathrm{NH}_{3}$ (e), $\mathrm{H}_{2}$ (f), $\mathrm{CH}_{4}$ (g) e $\mathrm{CO}_{2}$ (h) .... 92

Figura 28 - Perfil de concentração das espécies: inertes particulados (a), inertes solúveis (b), cálcio (c), lactato (d), lactato da fermentação (e), lactato da oxidação (f) e DQO (g) 93 


\section{LISTA DE TABELAS}

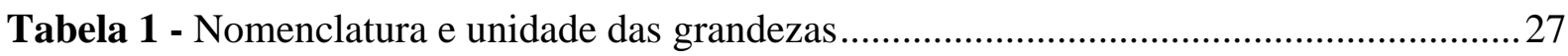

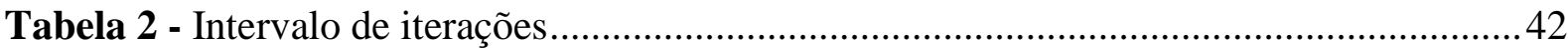

Tabela 3 - Parâmetros da cinética biológica: taxas de crescimento máximo ...........................44

Tabela 4 - Parâmetros adimensionais de cinética biológica: taxas de produção de biomassa 44

Tabela 5 - Parâmetros de cinética biológica: constantes de Monod ........................................4 44

Tabela 6 - Parâmetros de cinética biológica: taxas de decaimento (consumo) ....................... 45

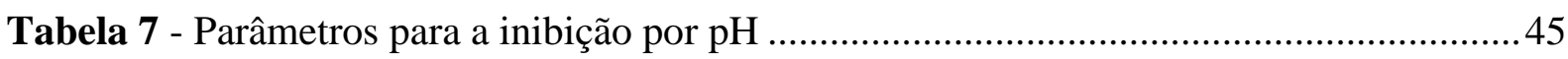

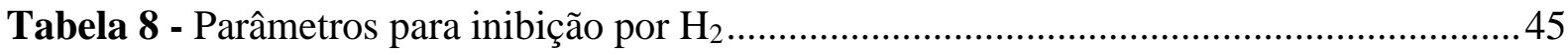

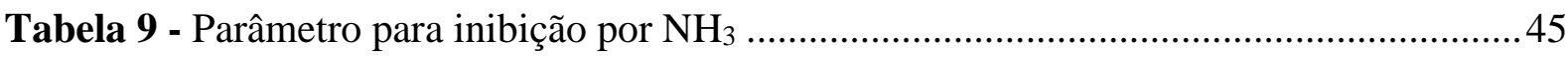

Tabela 10 - Parâmetro da quantidade de carbono nas variáveis de estado do modelo .............46

Tabela 11 - Parâmetro da quantidade de nitrogênio nas variáveis de estado do modelo .........46

Tabela 12 - Parâmetros estequiométricos: taxas de formação em reações bioquímicas ..........46

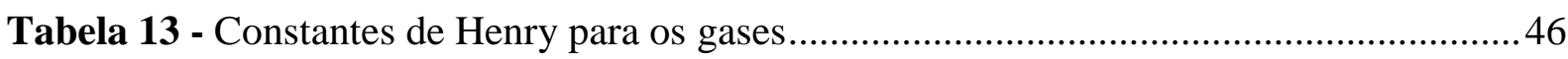

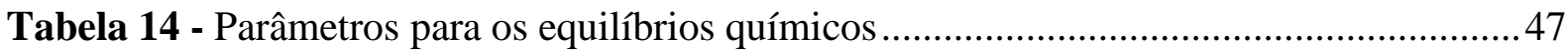

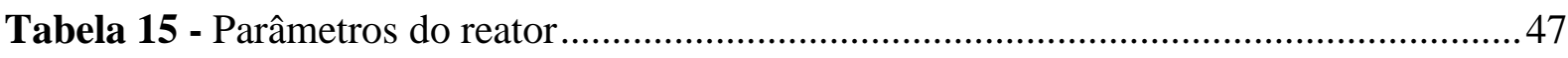

Tabela 16 - Parâmetros para simulação do modelo ADM1 completo (continua) .................... 48

Tabela 17 - Parâmetros para simulação do modelo ADM1 completo (conclusão) .................. 48

Tabela 18 - Nomenclatura e descrição das espécies consideradas ..........................................50

Tabela 19 - Valores adotados para as concentrações das diferentes espécies na alimentação e

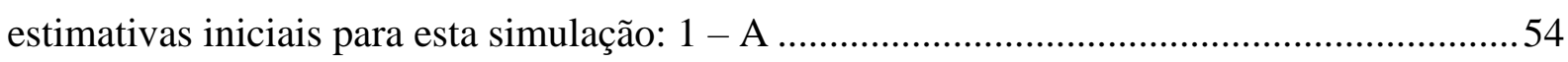

Tabela 20 - Estimativas iniciais das concentrações das diferentes espécies da Simulação 1-C 
Tabela 21 - Simulação feita com 80 iterações (continua)

Tabela 22 - Simulação feita com 800 iterações (continua) 76

Tabela 23- Simulação feita com 8.000 iterações (continua) ................................................ 78

Tabela 24 - Simulação feita com 80.000 iterações (continua)............................................... 80

Tabela 25 - Simulação feita com 800.000 iterações (continua)............................................... 82

Tabela 26 - Comparação entre as simulações feitas (1) neste trabalho e (2) Rosen e Jeppsson (2006) e Danielsson (2014) para as concentrações das diferentes espécies após 50 dias de tratamento 87

Tabela 27 - Tempo de CPU aproximado em função de cada número de iterações (n) 94 


\section{LISTA DE ABREVIATURAS, SIGLAS E SÍMBOLOS}

\section{Siglas}

ADM1 Anaerobic Digestion Model number 1

APBR Anaerobic packed bed reactor

DQO Demanda química de oxigênio

EDO Equações diferenciais ordinárias

IWA International Water Association

\section{Símbolos latinos}

$C_{i}$

Teor de carbono do componente $i$

$f_{\text {produto,substrato }}$

Rendimento (catabolismo apenas) do produto por substrato

Função de inibição

$k_{A / B, i} \quad$ Constante ácido-base para a componente $i$

$k_{\text {dec }}$

Taxa de decaimento de primeira ordem para a morte de biomassa

$k_{L} a$

Coeficiente de transferência gás-líquido

$k_{m}$

Velocidade máxima específica de consumo - Monod

$K_{a}$

Constante de equilíbrio ácido-base

$K_{H}$

Coeficiente da lei de Henry

$K_{I}$

Constante de inibição (50\% da concentração inibitória)

$K_{S}$

Constante Monod que corresponde à metade da velocidade máxima

$N_{i}$

Teor de carbono do componente $i$

$p_{\text {gas }}$

Pressão do gás

$p H$

Potencial hidrogeniônico $\left(-\log _{10}\left[\mathrm{~S}_{\mathrm{H}+}\right]\right)$

$p K_{a} \quad-\log _{10}\left[\mathrm{~K}_{\mathrm{a}}\right]$

$Q$

Vazão volumétrica

$R$

Constante universal (lei dos gases ideais)

$S_{i}$

Componente $i$ solúvel (variável dinâmica ou algébrica)

$S_{I} \quad$ Componente inibitório

$t \quad$ Tempo

T Temperatura

V Volume 
$X_{i} \quad$ Biomassa consumidora do componente particulado $i$

$Y_{\text {subs }} \quad$ Rendimento de biomassa no substrato

\section{Símbolos gregos}

$v_{i, j} \quad$ Coeficientes de taxa para cada componente $i$ no processo $j$

$\rho_{i, j} \quad$ Taxa do componente $i$ para o processo $j$

\section{Subscritos (componentes substratos e demais nomenclaturas)}

\begin{tabular}{ll}
$\mathrm{Aa}$ & Aminoácidos \\
$\mathrm{Ac}$ & Acetato \\
$\mathrm{ac}^{-}$ & Ácido acético \\
$\mathrm{Na}$ & Ânions \\
$\mathrm{Bu}$ & Butirato \\
$\mathrm{bu}^{-}$ & Ácido butírico \\
$\mathrm{Ca}$ & Cálcio \\
$\mathrm{Cat}$ & Cátions \\
$\mathrm{Ch}$ & Carboidratos \\
$\mathrm{ch} 4$ & Metano \\
$\mathrm{c} 4$ & Particulado de butirato e valerato \\
$\mathrm{Fa}$ & Ácidos graxos \\
$\mathrm{hco3}$ & Bicarbonato \\
$\mathrm{h} 2$ & Hidrogênio \\
$I$ & Indexação do componente/espécie \\
In & Condição de entrada do reator \\
$\mathrm{I}$ & Inertes \\
$\mathrm{IC}$ & Carbono inorgânico \\
$\mathrm{IN}$ & Nitrogênio inorgânico \\
$\mathrm{Lac}$ & Indexação do processo (Matrix Petersen) \\
lac,o & Pactato \\
\hline $\mathrm{Larticulado} \mathrm{de} \mathrm{lactato:} \mathrm{fermentação}$
\end{tabular}


Ms Monossacarídeos

nh3

Amônia

Out Condição de saída do reator

$\operatorname{Pr}$

Proteínas

Pro

Propionato

pro $^{-}$

Ácido propiônico

$\mathrm{Su}$

Açúcar

$\mathrm{Va}$

Valerato

$\mathrm{va}^{-}$

Ácido valérico

$\mathrm{Xc}$

Compósitos 


\section{SUMÁRIO}

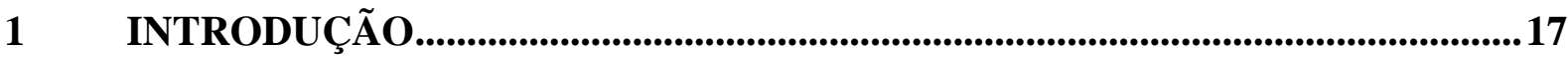

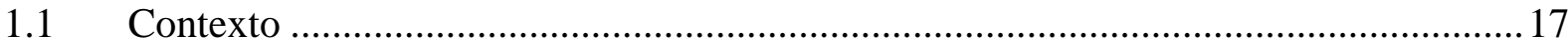

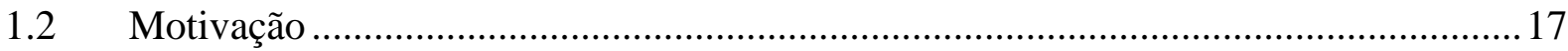

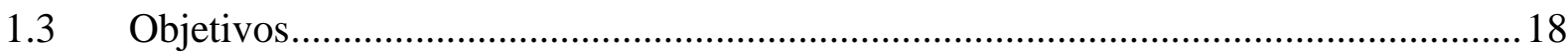

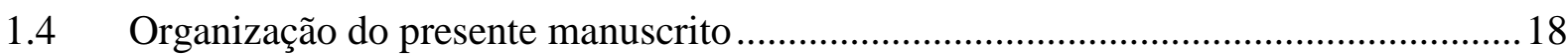

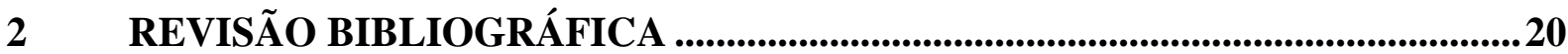

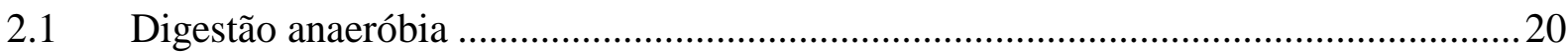

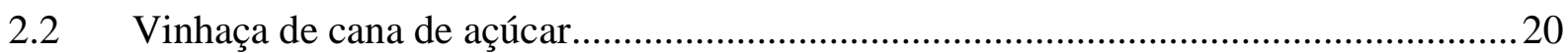

2.3 Biorreatores para tratamento anaeróbio de efluentes ....................................................21

2.4 Modelagem e simulação no tratamento anaeróbio de efluentes ....................................22

2.5 Modelo ADM1 para tratamento anaeróbio de efluentes ................................................22

2.6 Linguagem de programação Python ........................................................................24

3 TRATAMENTO ANAERÓBIO DE EFLUENTES: CARACTERIZAÇÃO E MODELAGEM MATEMÁTICA .............................................................................26

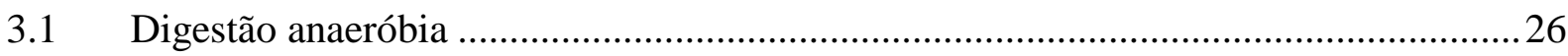

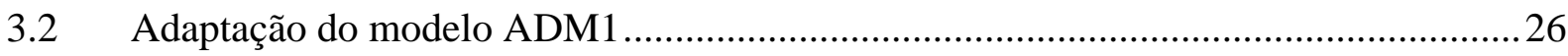

3.3 Equações do modelo ADM1 adaptado …………………………………………......... 30

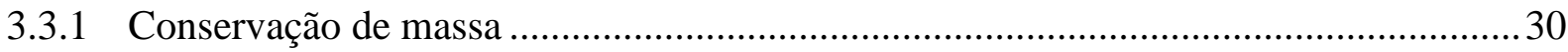

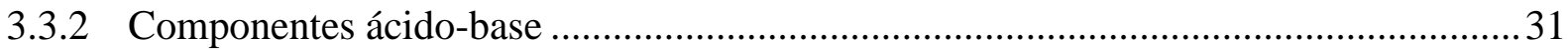

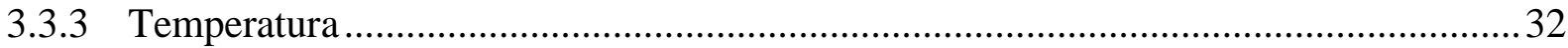

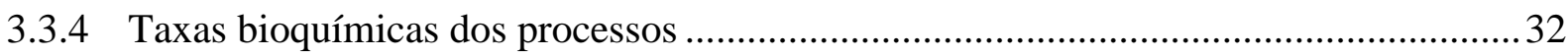

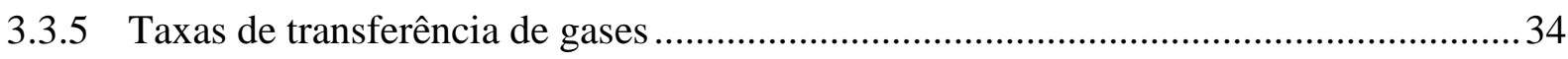

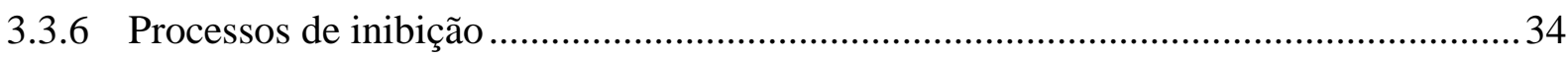

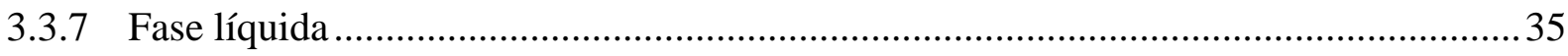

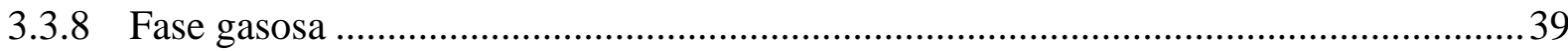




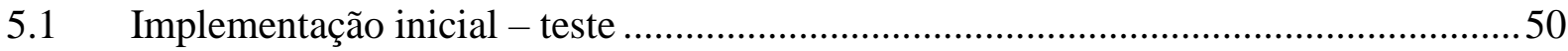

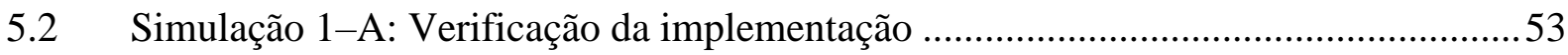

5.3 Simulação 1-B: Dados de entrada da Simulação 1-A mas 5 dias de tratamento ..........59

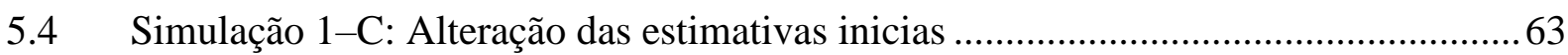

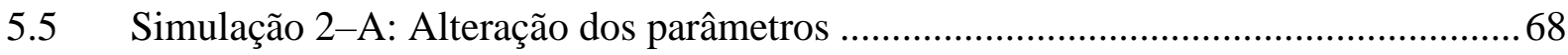

5.6 Simulação 2-B: Comparação de diferentes números de iteração.................................73

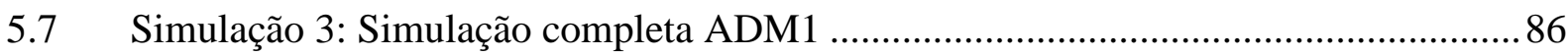

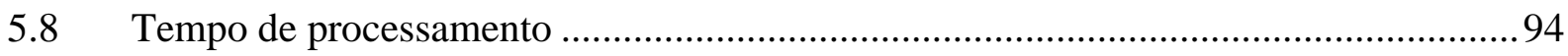

6 CONCLUS

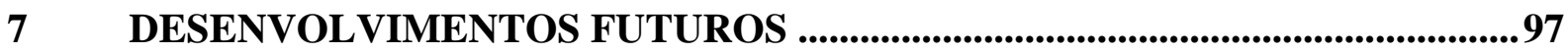

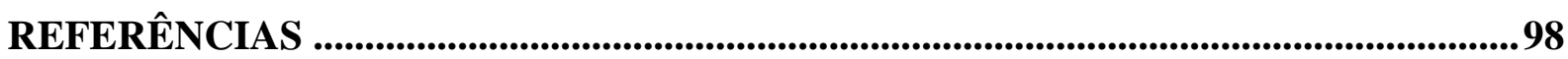

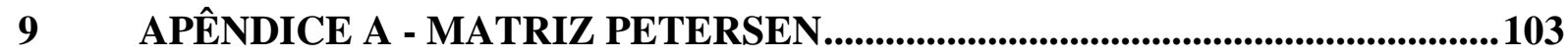




\section{INTRODUÇÃO}

\subsection{Contexto}

O controle da poluição é afetado por questões políticas, recursos econômicos, força institucional e aspectos culturais. Para a maioria dos países, a implantação de estações de tratamento de efluentes mostra-se como desafio (VON SPERLING, 2007).

O tratamento de efluentes por digestão anaeróbia tem sido de grande interesse para as agroindústrias e a implantação desta tecnologia tem crescido rapidamente ao redor do mundo. Dentre suas vantagens é possível destacar: menor produção de lodo, menor custo de implantação e operação, baixo custo de energia e, especialmente, a possibilidade de obtenção de gás metano como combustível (ABBASI et al., 2012).

A dinâmica dos fluidos computacional (CFD - computational fluid dynamics) pode ser fundamental para a engenharia de bioprocessos e biorreatores usados para o tratamento de efluentes na medida em que CFD é uma ferramenta para prever o comportamento de sistemas envolvendo fluidos (MALISKA, 1995). Assim, CFD pode rapidamente auxiliar na redução de custos envolvidos nos processos, na identificação de falhas ou em situações de emergência como também pode ser aplicada para conceber eventuais modificações do biorreator e, especialmente, para projetos de aumento de escala (scale-up).

O avanço dos recursos computacionais (hardware e software) tem possibilitado a concepção e o desenvolvimento de modelos matemáticos cada vez mais abrangentes com vistas à simulação de sistemas mais complexos de tratamento biológico de efluentes, em diferentes condições operacionais. Elaborado pela IWA (International Water Association), o modelo dinâmico de digestão anaeróbia ADM1(Anaerobic Digestion Model No. 1) é um dos mais abrangentes e, conforme discutido a seguir, tem sido utilizado com base para modelagem e simulação computacional de biorreatores para tratamento de efluentes.

\subsection{Motivação}

O modelo de digestão anaeróbia ADM1 tem sido amplamente utilizado desde sua publicação (BATSTONE et al., 2002). Muitos pesquisadores têm buscado alterar/adaptar o modelo original visando contornar problemas que surgiram conforme a necessidade de variáveis e/ou parâmetros em cenários específicos (DERBAL et al., 2009; JACOB, 2015; LÜBKEN et al., 2007; ROSEN; JEPPSSON, 2006). 
Para aproximar o modelo ADM1 fundamental (original) às particularidades dos processos anaeróbios (o que tende a possibilitar o conhecimento mais profundo destes processos), existem diferentes oportunidades de melhorias. Por exemplo, tais melhorias podem estar relacionadas às vias metabólicas intracelulares, aos mecanismos e interações entre os microrganismos anaeróbios em microescala e à representação multidimensional do modelo (GADEN, 2013; YU et al., 2013). Outras melhorias podem ser alcançadas através da simulação e avaliação dos diferentes parâmetros do modelo ADM1.

Ainda que a linguagem Python de programação esteja rapidamente se difundindo tanto entre cientistas quanto entre desenvolvedores de sistemas computacionais como uma alternativa promissora à outras linguagens, são escassos os trabalhos que implementaram o modelo ADM1 em Python. Por ser software livre e fácil de aprender, trata-se de opção interessante para iniciantes no campo da computação (SAYAMA, 2015). A programação do modelo ADM1 em linguagem Python (com seu amplo uso e disponibilidade gratuita) possibilitará futuras modificações e melhorias ao simulador de biorreatores para tratamento de efluentes agroindustriais que resultará do presente trabalho e que será disponibilizado na forma de manual ao grupo de pesquisas AGROENERBIO - Energia e Simulação na Engenharia de Biossistemas e no Agronegócio.

\subsection{Objetivos}

O presente trabalho tem como objetivo a modelagem computacional de biorreatores para tratamento anaeróbio de efluentes agroindustriais. Especificamente, foi desenvolvido um modelo dinâmico com base no modelo ADM1, com particular interesse à vinhaça de cana de açúcar, considerando as modificações propostas em Rosen e Jeppsson (2006) e adaptações feitas por Danielsson (2014).

O simulador numérico foi desenvolvido em linguagem Python de programação e foram feitas simulações para comparação e avaliação da eficiência do simulador. Também foi elaborado um manual do código de programação (em linguagem Python) para dar suporte ao grupo de pesquisas AGROENERBIO - Energia e Simulação na Engenharia de Biossistemas e no Agronegócio, com vistas a desenvolvimentos futuros do simulador.

\subsection{Organização do presente manuscrito}

Este manuscrito está organizado conforme descrito a seguir.

O capítulo 1 faz uma introdução ao objeto de pesquisa desta dissertação de mestrado. 
No capítulo 2 consta a revisão bibliográfica $(i)$ do processo de digestão anaeróbia e dos biorreatores mais utilizados neste tipo de tratamento, (ii) sobre modelagem e simulação numérica de processos de digestão anaeróbia, (iii) sobre o modelo ADM1 (incluindo breve descrição e aplicações) e (iv) sobre aplicações da linguagem Python de programação.

O capítulo 3 descreve o modelo matemático ADM1 para o processo de digestão anaeróbia, apresentando as respectivas equações governantes e as modificações feitas por Rosen e Jeppsson (2006) e Danielsson (2014). Trata-se do modelo que serviu como base para este trabalho.

O capítulo 4 apresenta a metodologia de implementação do modelo, contemplando um algoritmo do código de programação e uma descrição do embasamento das simulações.

No capítulo 5 são apresentados os resultados das simulações.

O capítulo 6 faz uma conclusão e o capítulo 7 apresenta possíveis melhorias futuras, encontradas ao longo do desenvolvimento deste trabalho de pesquisa.

Por fim, são apresentadas as referências bibliográficas utilizadas pela autora (deste trabalho de pesquisa). 


\section{REVISÃO BIBLIOGRÁFICA}

\subsection{Digestão anaeróbia}

O processo de tratamento anaeróbio é capaz de lidar com efluentes com alta carga orgânica e resistência de digestão, com baixa produção de lodos. Apresenta vantagens em relação ao tratamento aeróbio tais como produção de biogás, possibilidade de operar por longos períodos sem alimentação, e remoção fósforo da biomassa e minimização de problemas com algas (BRITTON et al., 2005; JORDAAN; ACKERMAN; CICEK, 2010).

A digestão anaeróbia tem sido usada para o tratamento de efluentes em diversos países. Em termos energéticos, o processo anaeróbio de tratamento de efluentes industriais de alta carga orgânica é capaz de produzir biogás, sendo mais vantajoso que o processo aeróbio (VERMA, 2012).

Demais vantagens do tratamento anaeróbio de efluentes incluem as reduções $(i)$ na área necessária para a planta, (ii) no custo operacional (uma vez que não é necessária a aeração), (iii) na emissão de $\mathrm{CO}_{2}$, (iv) na produção de lodo (LIU et al., 2007). Tais benefícios justificam a crescente implantação de sistemas anaeróbios de tratamento, frente ao alto custo da energia e a regulamentações ambientais cada vez mais estritas (SCHOEN, 2009).

\subsection{Vinhaça de cana de açúcar}

A vinhaça de cana de açúcar é um resíduo de grande importância e interesse para sustentabilidade da agroindústria no Brasil que é o principal país competidor na produção de bioetanol de cana. O destino mais comum deste resíduo é a aplicação direta no solo, na forma de fertilizante, por ser uma alternativa mais econômica para as agroindústrias. Entretanto, sabe-se que esta forma de disposição pode acarretar em contaminação no solo e lençol freático a níveis ambientalmente preocupantes (LYRA et al. 2003).

A digestão anaeróbia deste efluente agroindustrial mostra-se como uma alternativa interessante para evitar os riscos ambientais citados anteriormente, além das vantagens inerentes deste processo de digestão, como a possibilidade de geração de bioenergia.

A composição da vinhaça de cana de açúcar é dependente do processo produtivo e da matéria-prima empregada, mas normalmente este resíduo possui um elevado teor de matéria orgânica, com DQO de 50 a 150 g.L $\mathrm{L}^{-1}$, pH entre 3,5 e 5,0 e com odor desagradável para seres humanos (ESPAÑA-GAMBOA et al., 2011). 
Exemplos de pesquisas que têm sido feitas no Brasil para viabilizar e justificar o emprego do processo de digestão anaeróbia para tratamento de vinhaça foram apresentadas por Okiyama (2013) que caracterizou a hidrodinâmica de um biorreator de leito fixo através da modelagem matemática e por Ferraz (2013) que estudou a influência de diferentes sistemas/tipos de reatores e materiais suportes na digestão anaeróbia da vinhaça de cana de açúcar.

\subsection{Biorreatores para tratamento anaeróbio de efluentes}

Os tipos de reatores para tratamento anaeróbio são definidos, basicamente, em função da sua configuração, forma de crescimento de micro-organismos e faixa de temperatura de operação.

Nos reatores para o tratamento anaeróbio é desenvolvida uma comunidade de microrganismos específica que tem papel importante no tratamento (GRADY JR, 2011). A temperatura do reator é um dos mais importantes fatores físicos para seleção desta comunidade e influenciará no crescimento microbiano.

Fatores que interferem no projeto de sistemas de digestão anaeróbia incluem a temperatura, o tipo de mistura e a porcentagem de sólidos totais no efluente (KOSSEVA; WEBB, 2013). Dois tipos de reatores são usualmente empregados, com padrões de escoamento característicos, a saber: reator de fluxo contínuo de mistura perfeita (CSTR Continuously Stirred Tank Reactor) e reator pistonado (PFR - plug flow reactor).

O reator CSTR é mais usado para efluentes com baixo nível de sólidos (2 a 10\%), podendo ser aplicado para tratar resíduos orgânicos oriundos de cozinhas, estrume animal e esgoto (KOSSEVA; WEBB, 2013). Sendo de fácil operação, pode ser uma opção de baixo custo. Sua alimentação é feita de forma contínua, assim como o processo de mistura, que também se dá continuamente. Neste tipo de reator o tempo de retenção hidráulica é igual ao tempo de retenção celular, quando não há reciclo. Vega De Lille et al. (2016) utilizaram o modelo ADM1 como base para modelagem de um reator do tipo CSTR, com resultados satisfatórios.

O reator PFR opera com efluentes com mais de $10 \%$ de sólidos totais. Em geral, seu formato é cilíndrico, cujo comprimento é muito maior que o diâmetro. A alimentação também é contínua, porém não envolve processo de mistura e as etapas de hidrólise, acidogênese, acetogênese e metanogênese não ocorrem na mesma faixa de volume do reator (KOSSEVA; WEBB, 2013). Donoso-Bravo et al. (2018) alcançaram bons resultados ao implementarem o modelo ADM1 em um reator anaeróbio do tipo PFR. 


\subsection{Modelagem e simulação no tratamento anaeróbio de efluentes}

A simulação de sistemas multifásicos segue duas vertentes: correlações empíricas e modelos fenomenológicos (NAESSENS et al., 2012). Baseadas em medições e coleta de dados, as primeiras desenvolvem relações entre parâmetros importantes que precisam ser avaliados por dados experimentais, enquanto que os fenomenológicos se baseiam em eventos físicos, químicos e biológicos.

Se por um lado as correlações empíricas permitem alcançar excelentes resultados em curto prazo, elas não agregam fenômenos físicos e se comportam como "caixa preta", de modo que sua aplicação tende a ser limitada às condições utilizadas no experimento. Por levarem em consideração fenômenos importantes, os modelos fenomenológicos são mais versáteis e abrangentes. Contudo, eles requerem conhecimento mais profundo do processo.

Ainda que empregada há décadas em diversos campos da engenharia, a modelagem computacional (também chamada virtualização) é incipiente no setor agroindustrial (SAGUY, 2016). Modelos computacionais de digestão anaeróbia são pouco utilizados pelas indústrias, as quais têm usualmente recorrido a métodos empíricos (GADEN, 2013). Este fato pode ser atribuído à complexidade do processo de digestão anaeróbia em suas diferentes etapas (GADEN, 2013; YU et al., 2013). Não obstante, desde os trabalhos pioneiros (ANDREWS, 1968), a modelagem de biorreatores para tratamento anaeróbio tem sido aprimorada nos processos de interações e inibições microbianas (ANGELIDAKI; ELLEGAARD, 1999; BATSTONE et al., 2002; VAVILIN et al., 2007).

Para otimizar o processo em aspectos como dimensionamento do reator, qualidade do efluente, custos operacionais e produção de biogás, os métodos computacionais têm sido usados para simular mecanismos químicos, físicos e biológicos. Dentre os modelos complexos recentemente desenvolvidos, o modelo ADM1 - Anaerobic Digestion Model No. 1 (BATSTONE et al., 2002) tem sido amplamente referenciado (YU et al., 2013).

\subsection{Modelo ADM1 para tratamento anaeróbio de efluentes}

O modelo ADM1 foi desenvolvido por pesquisadores da IWA (International Water Association) que lidam com modelagem matemática de processos de digestão anaeróbia (BATSTONE et al., 2002). O objetivo foi elaborar um modelo universal e servir como base para modelagem, auxiliando assim no dimensionamento, na operação e no controle de reatores anaeróbios. De fato, vários pesquisadores têm usado o modelo ADM1 como base para criar outros modelos, por exemplo: 
- O modelo ADM1 tem sido desenvolvido em ambiente MATLAB/SIMULINK por pesquisadores na Universidade Lund (Suécia) para uso na plataforma BSM2 - Benchmark Simulation Model 2 (ROSEN; JEPPSSON, 2006), também criada pela IWA para modelagem e simulação de uma planta de tratamento de efluentes.

- Brouwer (2010) e McCrum (2012) desenvolveram um modelo ADM1 modificado em conjunto com o modelo DAIRES voltado para efluentes com estrume de vacas leiteiras.

- Utilizando MATLAB/SIMULINK, Boubaker e Ridha (2008) simularam o processo de codigestão anaeróbia mesofílica de águas residuárias da produção de azeite com resíduos sólidos da produção, com boa precisão na simulação dos parâmetros avaliados.

- Também usando MATLAB/SIMULINK, Chen et al. (2009) modelaram a digestão anaeróbia em dois estágios utilizando biorreatores CSTR e UASB (Upflow Anaerobic Sludge Blanket) em série para o tratamento de águas residuárias da produção de medicamento. O modelo mostrou-se adequado para simular o UASB mas apresentou falhas na simulação do CSTR. Os autores citaram como limitação a presença de etanol no efluente, convertida em ácido acético, que não foi considerada no modelo.

- Jurado, Gavala e Skiadas (2012) usaram o modelo ADM1 para prever o efeito da remoção de amoníaco aquoso sobre a eficiência da digestão anaeróbia de esterco de suínos e/ou fibras de estrume pré tratadas em biorreatores CSTR. O modelo mostrou-se mais adequado para simular os parâmetros cinéticos com esterco do que para os digestores alimentados com mistura de estrume e fibras pré tratadas, sendo necessário modificar os parâmetros cinéticos do ADM1.

- Lee e Lee (2013) aplicaram o modelo ADM1 para uma planta de tratamento municipal de águas residuárias na Coreia do Sul. A eficiência do modelo foi verificada mediante comparação dos valores simulados contra os correspondentes experimentais.

- Demais aplicações do modelo ADM1 para tratamento anaeróbio incluem o condensado do evaporador de fábrica de pasta de sulfito (SILVA et al., 2009), resíduos orgânicos com lodo ativado em estado mesófilo (DERBAL et al., 2009) e tratamento anaeróbio em duas etapas de águas residuárias de uma destilaria de etanol em trabalho pioneiro (TARUYANON; TEJASEN, 2010).

Devido ao grande número de parâmetros necessários ao modelo, pesquisas têm sido feitas visando facilitar a aplicação e a programação do ADM1, por exemplo:

- Bułkowska et al. (2015) programaram o chamado modelo ADM1xp para simular a codigestão anaeróbia de silagem de milho e esterco de gado. O modelo ADM1xp é uma 
modificação do ADM1 com a incorporação da concentração de produtos particulados provenientes da degradação da biomassa proposto por Wett, Eladawy e Ogurek (2009) e programado por IFAK (2009).

- Lauwers et al. (2015) avaliaram se a estimativa de parâmetros via modelo ADM1 se mostrava adequada. Utilizando equações diferenciais ordinárias (EDOs) para carbono e nitrogênio inorgânicos bem como as funções de Hill para inibição por pH (ROSEN; JEPPSSON, 2006), os resultados foram otimistas.

- Batstone et al. (2005) programaram o modelo ADM1 para reator plug-flow em uma dimensão utilizando Aquasim 2.1d e agrupando mais de uma simulação em série para discretizar o modelo em uma dimensão. Mu et al. (2008) e Tartakovsky et al. (2008) também desenvolveram modelo em uma dimensão, mas recorrendo a relações empíricas em vez de discretizar as variáveis.

Yu et al. (2013) apontam que o modelo ADM1 pode incorporar melhorias não apenas relacionadas às vias metabólicas intracelulares e interações entre microrganismos anaeróbios em micro escala, mas também quanto à fluidodinâmica para maior conhecimento de fenômenos de transporte no interior dos reatores. Gaden (2013) programou o modelo ADM1 em três dimensões via método dos volumes finitos junto com CRAFTS (Coupled ReactionAdvenction-Flow Transient Solver), integrando o modelo bioquímico à dinâmica dos fluidos, o que permitiu inter-relações entre as variáveis até então desconhecidas.

Gaden (2013) concluiu que a robustez numérica é uma limitação do CRAFTS na simulação do modelo devido à baixa densidade da mistura, ao baixo gradiente de velocidade, ao baixo gradiente de concentração e à impraticabilidade de algumas equações bioquímicas em função da variação da concentração de algumas espécies químicas. Porém, possibilitou simular processos físicos que o ADM1 não é capaz. Haja vista as diferenças encontradas entre os resultados numéricos e os dados apresentados na literatura, o autor enfatiza a necessidade de mais estudos sobre a modelagem da digestão anaeróbia levando-se em conta a dependência em coordenadas espaciais (além do tempo).

\subsection{Linguagem de programação Python}

A linguagem Python é uma alternativa para o MATLAB, o qual é um software para realizar cálculos numéricos com matrizes. Dentre as vantagens da linguagem Python em relação ao MATLAB é possível citar a facilidade na instalação e as bibliotecas de funções numéricas (numpy), científicas (scipy) e de plotagem (matplotlib) que proporcionam muito do 
que é requisitado em engenharia. A extensão numpy transformou Python em ferramenta de programação numérica eficiente e fácil de ser utilizada (LUTZ, 2013). Demais vantagens da linguagem Python são discutidas em (RAYMOND, 2000).

Diferentemente do MATLAB, trata-se de uma linguagem gratuita que não necessita de licença, sendo totalmente livre para uso e distribuição. Embora apresente muitas semelhanças com o MATLAB (tais como a declaração de variáveis, a sintaxe simples e visualização), o Python é superior em alguns pontos. Compatível com sistemas Linux, Macintosh e Microsoft Windows, Python é muito estável e robusta, considerada uma das linguagens de programação atualmente mais utilizadas no mundo. Grandes empresas como Google e YouTube fazem uso desta linguagem, além de The Dropbox, The Raspberry, Netflix e NASA (LUTZ, 2013). Outras vantagens em relação ao MATLAB são (LANGTANGEN, 2006): a presença de uma caixa de ferramentas completa (muitas funções podem estar em um único arquivo), a facilidade de integração do ambiente Python com ferramentas externas (como C, C++, Fortran e até mesmo com MATLAB) e os recursos para programação orientada a objetos são mais desenvolvidos.

Chai (2008) desenvolveu uma interface em Python e MATLAB para simular modelos de plantas de tratamento de efluentes de lodo ativado visando mais flexibilidade na representatividade do modelo através da programação orientada a objetos. Concluiu que ambas interfaces desenvolvidas forneceram algoritmos poderosos para o controle avançado do sistema. Faria et al. (2015) também utilizaram a linguagem de programação Python em melhorias para plantas de tratamento de efluentes.

A linguagem Python tem se mostrado uma ferramenta importante para integração e controle de processo em diversas áreas. Bernard (2015) mostra a aplicação no campo da astronomia, Meyers e Sethna (2008) discutem as vantagens da linguagem para ciência da computação e engenharia, enquanto que Loksha et al. (2009), Pertusi et al. (2014) e Holmqvist e Sellberg (2016) citam o uso da linguagem Python na área de engenharia química.

Uma desvantagem relatada refere-se ao suporte técnico e à documentação do Python, que não são tão bons quanto a de outras linguagens, uma vez que Python é sem fins lucrativos (CHAI, 2008). Assim, faz-se necessário um estudo de forma independente para aprendizado e utilização desta linguagem.

Para resolução de equações diferenciais ordinárias, o Python dispõe da função 'odeint' da biblioteca 'scipy', usualmente empregada. Tal função tem uma rotina de integração para resolução das equações diferenciais ordinárias que utiliza métodos de comprimento de passo variável e cálculo de erro para retornar valores precisos. 


\section{TRATAMENTO ANAERÓBIO DE EFLUENTES: CARACTERIZAÇÃO E MODELAGEM MATEMÁTICA}

\subsection{Digestão anaeróbia}

Ocorrendo na ausência de oxigênio e composto por vários estágios, a digestão anaeróbia é um processo bioquímico em que a matéria orgânica é convertida por microrganismos numa série de reações, tendo como produto final gás metano e dióxido de carbono. A digestão anaeróbia pode ser dividida em quatro etapas, descritas a seguir.

A primeira etapa é (hidrólise) é o processo bioquímico em que carboidratos, proteínas e lipídios são respectivamente quebrados em monossacarídeos solúveis, aminoácidos e ácidos graxos de cadeia longa e glicerol. Conduzido por diversos micro-organismos e enzimas extracelulares, este processo é comumente representado por cinéticas de primeira ordem (BATSTONE et al., 2002). Esta etapa é a mais lenta do processo de digestão anaeróbia.

A etapa seguinte é a acidogênese em que monômeros resultantes da etapa anterior são absorvidos por bactérias e degradados em moléculas mais simples como ácidos orgânicos de cadeia curta, álcoois, hidrogênio e dióxido de carbono. Este processo não requer um aceptor ou doador de elétrons, diferentemente da acetogênese.

$\mathrm{Na}$ próxima etapa (acetogênese) os ácidos graxos e álcoois são decompostos para formar hidrogênio assim como dióxido de carbono e ácido acético. Diferentemente dos anteriores, este processo envolve um número mais restrito de microrganismos e reações. Além disto, tais reações necessitam de aceptores de elétrons que agem como inibidores para o próprio processo da acetogênese devido ao aumento na quantidade de hidrogênio livre.

Durante a metanogênese (última etapa da digestão anaeróbia) ocorre a produção do gás metano. Este componente principal do biogás é produzido por reações consumindo o ácido acético ou hidrogênio das fases anteriores.

\subsection{Adaptação do modelo ADM1}

O modelo matemático considerado no presente trabalho tem por base mecanismos químicos, físicos e biológicos que ocorrem na digestão anaeróbia. Em seu atual estágio de desenvolvimento, o modelo conta com 41 parâmetros estequiométricos, 36 parâmetros bioquímicos e 23 parâmetros físico-químicos. A Tabela 1 descreve a nomenclatura e unidades das grandezas utilizadas no modelo ADM1, o qual usa a demanda química de oxigênio (DQO) como base de cálculo. 
Tabela 1 - Nomenclatura e unidade das grandezas

\begin{tabular}{|c|c|c|}
\hline Símbolo & Descrição & Unidades \\
\hline$C_{i}$ & Teor de carbono do componente $i$ & $\mathrm{kmol}-\mathrm{C} \cdot \mathrm{kg}-\mathrm{DQO}$ \\
\hline$I$ & $\begin{array}{l}\text { Indexação do componente (Matriz Petersen - } \\
\text { Apêndice A) }\end{array}$ & Adimensional \\
\hline$I$ & Função de inibição & Adimensional \\
\hline$J$ & $\begin{array}{l}\text { Indexação do processo (Matrix Petersen - Apêndice } \\
\text { A) }\end{array}$ & Adimensional \\
\hline$k_{A / B, i}$ & Constante ácido-base para a componente $i$ & $\mathrm{M}^{-1} \cdot \mathrm{d}^{-1}$ \\
\hline$k_{d e c}$ & $\begin{array}{l}\text { Taxa de decaimento de primeira ordem para a morte de } \\
\text { biomassa }\end{array}$ & $\mathrm{d}^{-1}$ \\
\hline$k_{L a}$ & Coeficiente de transferência gás-líquido & $\mathrm{d}^{-1}$ \\
\hline$k_{m}$ & $\begin{array}{l}\text { Velocidade máxima específica de consumo de } \\
\text { substrato - Monod }\end{array}$ & $\mathrm{kg}-\mathrm{DQO} \cdot \mathrm{m}^{-3}{ }_{-} \mathrm{S} \cdot \mathrm{kg}-\mathrm{DQO} \cdot \mathrm{m}^{-3} \mathrm{X} \cdot \mathrm{d}^{-1}$ \\
\hline$K_{a}$ & Constante de equilíbrio ácido-base & $\mathrm{M}\left(\mathrm{kmol} \cdot \mathrm{m}^{-3}\right)$ \\
\hline$K_{H}$ & Coeficiente da lei de Henry & M.bar ${ }^{-1}$ \\
\hline$K_{I}$ & Constante de inibição & $\mathrm{kg}-\mathrm{DQO} \cdot \mathrm{m}^{-3}$ \\
\hline$K_{S}$ & $\begin{array}{l}\text { Concentração de substrato (corresponde à metade da } \\
\text { velocidade máxima) - Monod }\end{array}$ & $\mathrm{kg}-\mathrm{DQO} \cdot \mathrm{m}^{-3}$ \\
\hline$N_{i}$ & Concentração de nitrogênio do componente $I$ & $\mathrm{kmol}-\mathrm{N} \cdot \mathrm{kg}-\mathrm{DQO}{ }^{-1}$ \\
\hline$p_{\text {gas }}$ & Pressão do gás & bar \\
\hline$p H$ & $-\log _{10}\left[\mathrm{~S}_{\mathrm{H}+}\right]$ & adimensional \\
\hline$p K_{a}$ & $-\log _{10}\left[\mathrm{~K}_{\mathrm{a}}\right]$ & adimensional \\
\hline$Q$ & Vazão volumétrica & $\mathrm{m}^{3} \cdot \mathrm{d}^{-1}$ \\
\hline$S_{i}$ & Componente $i$ solúvel (variável dinâmica ou algébrica) & $\mathrm{kg}-\mathrm{DQO} \cdot \mathrm{m}^{-3}$ \\
\hline$S_{I}$ & componente inibitório & $\mathrm{kg}-\mathrm{DQO} \cdot \mathrm{m}^{-3}$ \\
\hline$T$ & Tempo & d (dia) \\
\hline$T$ & Temperatura & $\mathrm{K}$ \\
\hline$V$ & Volume & $\mathrm{m}^{3}$ \\
\hline$X_{i}$ & Componente particulado $i$ & $\mathrm{~kg}-\mathrm{DQO} \cdot \mathrm{m}^{-3}$ \\
\hline$Y_{\text {subs }}$ & Rendimento de biomassa no substrato & kg-DQO_X·kg-DQO_S ${ }^{-1}$ \\
\hline$v_{i, j}$ & Coeficientes de taxa para a componente $i$ no processo $j$ & $\mathrm{~kg}-\mathrm{DQO} \cdot \mathrm{m}^{-3}$ \\
\hline$f_{\text {prod,subs }}$ & $\begin{array}{l}\text { Rendimento (catabolismo apenas) do produto no } \\
\text { substrato }\end{array}$ & $\begin{array}{l}\text { kg-DQO_produto } \cdot k g- \\
\text { DQO_substrato } \\
-1\end{array}$ \\
\hline$\rho_{i}$ & Taxa para o processo $j$ & $\mathrm{~kg}-\mathrm{DQO} \mathrm{m}^{-3}$ \\
\hline
\end{tabular}

Fonte: Adaptado de BATSTONE, D. J. et al. The IWA anaerobic digester model no.1 (ADM1). Water Science and Technology, Oxford, v. 45, n. 10, p. 65-73, 2002. 
O biorreator considerado é do tipo CSTR com uma única entrada, uma única saída e volume constante. Todas as reações bioquímicas extracelulares foram consideradas de $1^{\text {a }}$ ordem assim como a taxa de decaimento da biomassa, ou seja, limitadas pela concentração do substrato. A cinética de consumo de substrato usada para reações bioquímicas intracelulares foi baseada no modelo Monod. O sistema de digestão anaeróbia baseado no modelo ADM1 é descrito através da matriz Petersen (apresentada no Apêndice A).

A ideia foi modelar as diferentes etapas (anteriormente descritas) que levam à decomposição da matéria orgânica até os produtos finais da digestão anaeróbia. Inicialmente foram consideradas, para este trabalho, as rotas metabólicas mais relevantes para o processo de digestão anaeróbia da vinhaça de cana de açúcar, que tem sido o substrato alvo nas pesquisas do grupo AGROENERBIO - Energia e Simulação na Engenharia de Biossistemas e no Agronegócio. Por ser a mais lenta e, por consequência, comprometer a evolução numérica da solução, a etapa de hidrólise foi desconsiderada nas primeiras simulações. Assim, foram considerados apenas os processos e componentes da acidogênese, acetogênese e metanogênese a partir de açúcares (glicose, conforme considerado no modelo ADM1) em rotas específicas, representadas por setas verdes no esquema da Figura 1. Após tal avaliação, partiu-se para a modelagem completa do ADM1. 
Figura 1 - Esquema dos componentes e processos da digestão anaeróbia baseada no modelo ADM1 Matéria Orgânica Complexa

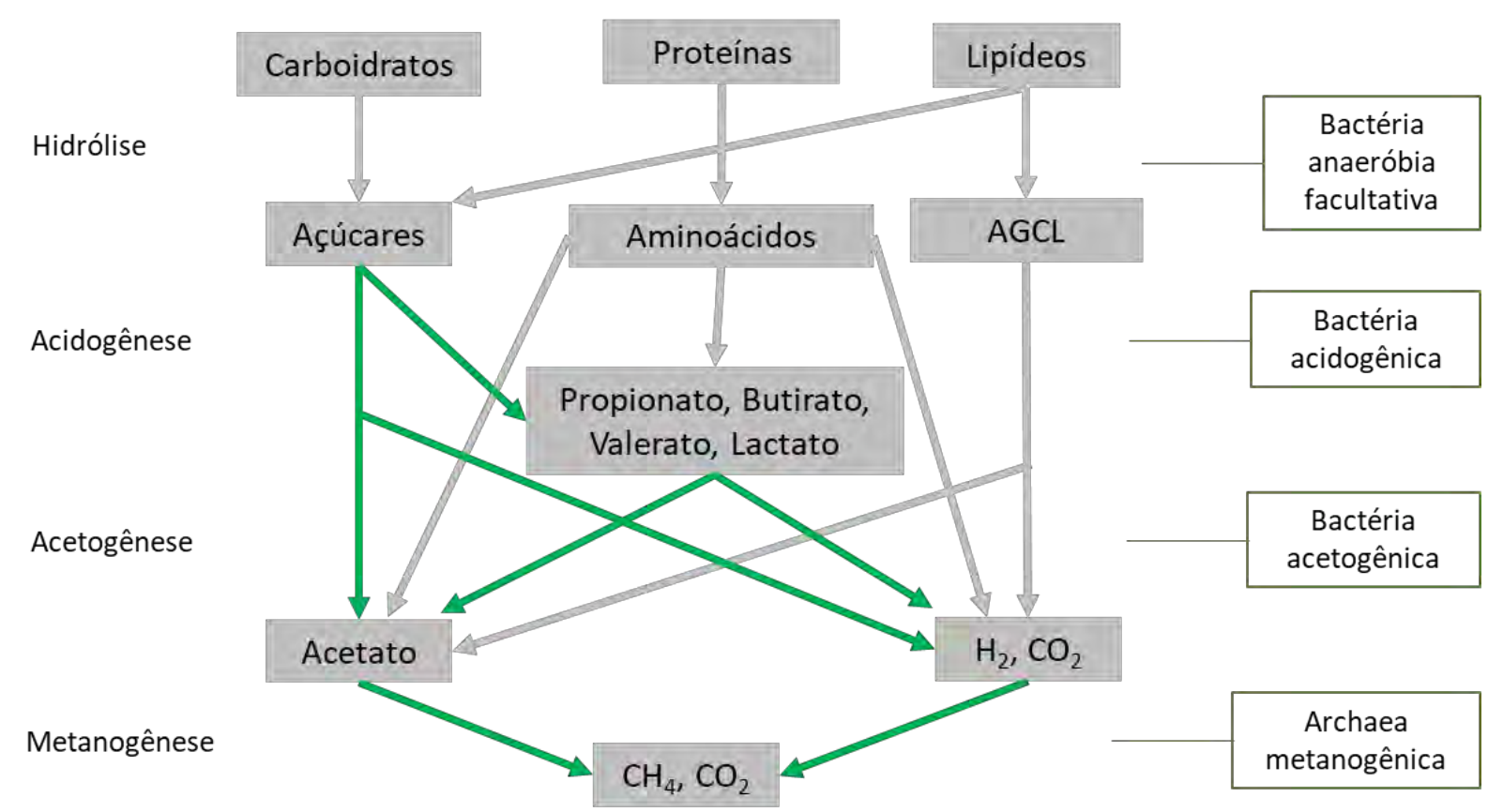

Fonte: SCHOEN, M. Numerical modelling of anaerobic digestion processes in agricultural biogas plants dissertation. 2009. 139 p. Dissertation (Doktor) - Fakultät Für Bauingenieurwissenschaften, Universität Innsbruck, Innsbruck, 2009.

A Figura 1 descreve o sistema de digestão anaeróbia por completo, em que as rotas destacadas pelas setas verdes foram consideradas nas primeiras simulações deste trabalho. Estas rotas descrevem o processo de digestão anaeróbia baseado em 16 reações da fase líquida e processos físico-químicos: equilíbrio ácido base entre os componentes do sistema e a transferência de massa entre as fases gasosa e líquida de $\mathrm{CO}_{2}, \mathrm{CH}_{4}$ e $\mathrm{H}_{2}$, conforme as equações em (ROSEN; JEPPSSON, 2006), descritas a seguir, e contemplando as adaptações em (DANIELSSON, 2014).

Na programação do ADM1, adaptada por Rosen e Jeppsson (2006), a inibição de pH é descrita por funções contínuas em vez de valores determinados, o desequilíbrio de nitrogênio e carbono é acertado (ajustado conforme as equações 7 e 8 apresentadas no item 3.3.1) e o rápido consumo de hidrogênio é modelado (e programado) como uma equação algébrica em vez de uma equação diferencial. Danielsson (2014) incluiu o substrato lactato e considerou a precipitação de cálcio, devido à sua influência na pressão parcial de dióxido de carbono. 


\subsection{Equações do modelo ADM1 adaptado}

A seguir são apresentadas as equações do modelo ADM1 adaptado por Rosen e Jeppsson (2006), com as inclusões feitas por Danielsson (2014). Estas equações serviram como base para o desenvolvimento do presente trabalho.

\subsubsection{Conservação de massa}

A base para modelagem é o balanço de massa no reator expressa por:

$$
\frac{d m_{\mathrm{var}}}{d t}=\dot{m}_{\mathrm{var}, \text { in }}-\dot{m}_{\mathrm{var}, \text { out }}+\dot{m}_{\mathrm{var}, \text { reac }}
$$

Para uma dada espécie 'var', $m_{\text {var }}$ é sua massa, $\dot{m}_{\text {var }}$ refere-se à vazão mássica de entrada (subscrito 'in') ou saída (subscrito 'out') enquanto que o subscrito 'reac' se refere à produção (ou consumo) nas reações.

Considerando fluido incompressível tem-se:

$$
V_{\text {liq }} \frac{d S_{\mathrm{var}}}{d t}=q_{\text {in }} S_{\text {var,in }}-q_{\text {in }} S_{\text {var,out }}+V_{\text {liq }} r_{\mathrm{var}}
$$

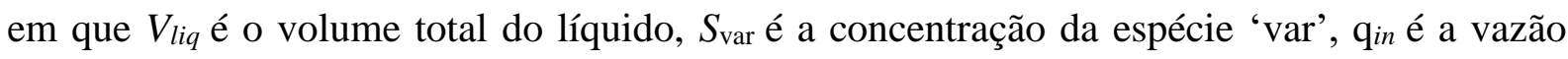
volumétrica do líquido e $r_{\mathrm{var}}$ é o termo fonte ou sumidouro (volume da espécie 'var' gerado ou consumido durante o processo). Considerando mistura perfeita no reator, as concentrações de saída são iguais às concentrações em qualquer ponto do reator. Assim, a equação (2) se torna:

$$
\frac{d S_{\mathrm{var}}}{d t}=\frac{q_{\text {in }}}{V_{\text {liq }}}\left(S_{\mathrm{var}, \mathrm{in}}-S_{\mathrm{var}}\right)+r_{\mathrm{var}-}
$$

sendo $S_{\text {var }}$ a variável desconhecida, $S_{\text {var,in }}$ seu valor estabelecido pela condição de contorno (na entrada) e $r_{v a r}$ a soma da contribuição de todos os processos. Esta soma é definida por:

$$
r_{\text {var- }}=\sum_{j}^{N} v_{i j} \rho_{j}
$$

em que $v$ representa a taxa bioquímica para os processos $j$ e componentes $i$ enquanto que $\rho$ representa a velocidade cinética da reação para cada processo $j$ com base na matriz Petersen (Apêndice A).

Pelo princípio de conservação de massa, uma unidade de massa (em base DQO) da matéria complexa $X_{C}$ resultará, após o processo de desintegração, em vários compostos de acordo com:

$$
\begin{aligned}
f_{s I, x c} S_{I}+f_{x I, x c} X_{I}+f_{c h, x c} X_{c h}+f_{p r, x c} X_{p r}+f_{l i, x c} X_{l i} & =0,1 S_{I}+0,25 X_{I}+0,2 X_{c h}+ \\
& +0,2 X_{p r}+0,25 X_{l i}
\end{aligned}
$$


A soma dos coeficientes empíricos dos rendimentos (determinados experimentalmente) deve ser unitária, ou seja:

$$
f_{s I, x c}+f_{x I, x c}+f_{c h, x c}+f_{p r, x c}+f_{l i, x c}=1
$$

Com base nos parâmetros determinados por Batstone et al. (2002), Rosen e Jeppsson (2006) calcularam a desintegração do nitrogênio conforme:

$$
\begin{aligned}
N I 0,1 S I+N I 0,25 X I+N_{c h} 0,2 X_{c h}+N_{a a} 0,2 X_{p r}+N_{l i} 0,25 X_{l i} & =0,0002+0,0005+0,0014 \\
& =0,0021
\end{aligned}
$$

Pelo cálculo acima nota-se que $0,0021 \mathrm{kmol}$ de nitrogênio é gerado na desintegração de cada $\mathrm{kg}$-DQO. Entretanto, foi proposta a geração de $0,002 \mathrm{kmol}-\mathrm{N} / \mathrm{kg}$, o que representa $5 \%$ a mais (ROSEN; JEPPSSON, 2006).

Rosen e Jeppsson (2006) adotaram as seguintes modificações:

- $f_{x I, x c}=0,2$ e $f_{l i, x c}=0,3$;

- $N I=0,06 / 14 \approx 0,00429 \mathrm{kmol}-\mathrm{N} / \mathrm{kg}-\mathrm{DQO}$;

- $N_{x c}=0,0376 / 14 \approx 0,00269 \mathrm{kmol}-\mathrm{N} / \mathrm{kg}-\mathrm{DQO}$;

- Inclusão na matriz Petersen de termos estequiométricos, por exemplo $\left(N_{b a c}-N_{x c}\right)$ e $\left(C_{b a c}-\right.$ $C_{x c}$ ), para rastrear excessos de nitrogênio e carbono, respectivamente, resultantes do decaimento da biomassa em compósito. Esta inclusão, não adicionada no modelo fornecido porém já sugerida por Batstone et al. (2002), também foi feita para os demais processos relacionados ao carbono e nitrogênio inorgânico.

Com a adoção das considerações acima, após desintegração tem-se (em kmol-C/kg-DQO):

$$
0,03 \times 0,1 S_{I}+0,03 \times 0,2 X_{I}+0,0313 \times 0,2 X_{c h}+0,03 \times 0,2 X_{p r}+0,022 \times 0,3 X_{l i}=0,02786
$$

Comparando com o valor proposto no modelo original ADM1 (0,03 kmol-C/kgDQO), há redução no conteúdo de carbono que pode se transformar em carbono inorgânico e possivelmente contribuir para a produção de dióxido de carbono no final do processo, se o modelo estiver atualizado. Assim, é sugerido usar o valor de $0.02786 \mathrm{kmol}-\mathrm{C} / \mathrm{kg}-\mathrm{DQO}$ para o conteúdo de carbono do material (ROSEN; JEPPSSON, 2006).

\subsubsection{Componentes ácido-base}

Por facilidade de entendimento e benefícios numéricos, Rosen e Jeppsson (2006) programaram as equações diferenciais ordinárias (EDOs) tomando por base o componente total e um dos componentes ácido-base (ao invés de definirem EDOs para cada um dos componentes ácido e básico). As equações dos componentes ácido-base são as seguintes: 


$$
\begin{aligned}
& \rho_{A, 4}=k_{A, B v a}\left[S_{v a-}\left(K_{a, v a}+S_{H+}\right)-K_{a, v a} S_{v a}\right] \\
& \rho_{A, 5}=k_{A, B b u}\left[S_{b u^{-}}\left(K_{a, b u}+S_{H+}\right)-K_{a, b u} S_{b u}\right] \\
& \rho_{A, 6}=k_{A, B p r o}\left[S_{p r o}\left(K_{a, p r o}+S_{H+}\right)-K_{a, p r o} S_{p r o}\right] \\
& \left.\rho_{A, 7}=k_{A, B a c}\left[S_{a c^{-}}\left(K_{a, a c}+S_{H+}\right)-K_{a, a c} S_{a c}\right)\right] \\
& \left.\rho_{A, 10}=k_{A, B c o 2}\left[S_{h c o 3}\left(K_{a, c o 2}+S_{H+}\right)-K_{a, c o 2} S_{I C}\right)\right] \\
& \left.\rho_{A, 11}=k_{A, B I N}\left[S_{n h 3}\left(K_{a, I N}+S_{H+}\right)-K_{a, I N} S_{I N}\right)\right]
\end{aligned}
$$

\subsubsection{Temperatura}

Rosen e Jeppsson (2006) consideraram os parâmetros físico-químicos $K_{w}, K_{a, c o 2}, K_{a, I N}$, $K_{H, c o 2}, K_{H, c h 4}, K_{H, h 2}$ e $p_{\text {gas,h2o }}$ como dependentes da temperatura de forma a permitir simulações em diferentes temperaturas.

\subsubsection{Taxas bioquímicas dos processos}

As equações a seguir regem as concentrações das diferentes espécies no reator.

$$
\begin{aligned}
& \rho_{1}=k_{d i s} \cdot X_{c} \\
& \rho_{2}=k_{h y d, c h} \cdot X_{c h} \\
& \rho_{3}=k_{h y d, p r} \cdot X_{p r} \\
& \rho_{4}=k_{h y d, l i} \cdot X_{l i} \\
& \rho_{5}=k_{m, s u} \cdot \frac{S_{s u}}{K_{S, s u}+S_{s u}} \cdot X_{s u} \cdot I_{5} \\
& \rho_{6}=k_{m, a a} \cdot \frac{S_{a a}}{K_{S, a a}+S_{a a}} \cdot X_{a a} \cdot I_{6} \\
& \rho_{7}=k_{m, f a} \cdot \frac{S_{f a}}{K_{S, f a}+S_{f a}} \cdot X_{f a} \cdot I_{7} \\
& \rho_{8}=k_{m, c 4} \cdot \frac{S_{v a}}{K_{S, c 4}+S_{v a}} \cdot X_{c 4} \cdot \frac{S_{v a}}{S_{b u}+S_{v a}+10^{-6}} \cdot I_{8} \\
& \rho_{9}=k_{m, c 4} \cdot \frac{S_{b u}}{K_{S, c 4}+S_{b u}} \cdot X_{c 4} \cdot \frac{S_{b u}}{S_{v a}+S_{b u}+10^{-6}} \cdot I_{9} \\
& \rho_{10}=k_{m, p r} \cdot \frac{S_{p r o}}{K_{S, p r o}+S_{p r o}} \cdot X_{p r o} \cdot I_{10} \\
& \rho_{11}=k_{m, a c} \cdot \frac{S_{a c}}{K_{S, a c}+S_{a c}} \cdot X_{a c} \cdot I_{11}
\end{aligned}
$$




$$
\begin{aligned}
& \rho_{12}=k_{m, h 2} \cdot \frac{S_{h 2}}{K_{S, h 2}+S_{h 2}} \cdot X_{h 2} \cdot I_{12} \\
& \rho_{13}=k_{d e c, X s u} \cdot X_{s u} \\
& \rho_{14}=k_{d e c, X a a} \cdot X_{a a} \\
& \rho_{15}=k_{d e c, X f a} \cdot X_{f a} \\
& \rho_{16}=k_{d e c, X c 4} \cdot X_{c 4} \\
& \rho_{17}=k_{d e c, X p r o} \cdot X_{p r o} \\
& \rho_{18}=k_{d e c, X a c} \cdot X_{a c} \\
& \rho_{19}=k_{d e c, X h 2} \cdot X_{h 2}
\end{aligned}
$$

Rosen e Jeppsson (2006) adicionaram uma pequena constante $\left(10^{-6}\right)$ nas equações das variáveis $\rho_{8}$ e $\rho_{9}$ para evitar divisão por zero. Das taxas bioquímicas citadas acima, foram consideradas na implementação inicial feita neste trabalho apenas as equações (19), (22) a (27) e (30) a (33). Conforme descrito anteriormente, foram excluídas as equações envolvidas no processo de hidrólise, considerando-se apenas a acidogênese, acetogênese e metanogênese de açúcares.

Adicionalmente foram incluídas as equações a seguir, contemplando as concentrações de lactato e cálcio, com base no trabalho de Danielsson (2014). O consumo de lactato foi implementado com base nos processos de oxidação e fermentação, assim como a taxa de decaimento da biomassa que foi implementada separadamente, para estes dois processos, conforme equações descritas a seguir:

$$
\begin{aligned}
& \rho_{20}=k_{\text {m,lacf }} \cdot \frac{S_{\text {lac }}}{K_{S, \text { lacf }}+S_{\text {lac }}} \cdot X_{\text {lacf }} \cdot I_{20} \\
& \rho_{21}=k_{\text {m,laco }} \cdot \frac{S_{\text {lac }}}{K_{S, \text { laco }}+S_{\text {lac }}} \cdot X_{\text {laco }} \cdot I_{21} \\
& \rho_{22}=k_{\text {dec }, X_{\text {lacf }}} \cdot X_{\text {lacf }} \\
& \rho_{23}=k_{\text {dec }, X_{\text {laco }}} \cdot X_{\text {laco }}
\end{aligned}
$$

A taxa de reação do cálcio é implementada conforme a equação seguinte, sendo o processo de precipitação: 


$$
\rho_{24}= \begin{cases}0 & : S_{c a}, S_{h c o 3}-<0 \\ K_{r, c a c 03} \cdot\left(\sqrt{S_{c a} \cdot S_{h c 03-}}-K_{S, p, c a c o 3}\right)^{2} & : S_{c a}, S_{h c o 3}-\geq 0\end{cases}
$$

Vale ressaltar que na ausência de um dos substratos, dos citados acima que foram adicionados ao modelo proposto por Rosen e Jeppsson (2006), o processo de simulação não é afetado, uma vez que tais concentrações podem ser definidas como zero.

\subsubsection{Taxas de transferência de gases}

As transferências de gases são modeladas pelas seguintes equações:

$$
\begin{aligned}
& \rho_{T, 8}=k_{L} a\left(S_{h 2}-16 \cdot K_{H, h 2} p_{g a s, h 2}\right) \\
& \rho_{T, 9}=k_{L} a\left(S_{c h 4}-64 \cdot K_{H, c h 4} p_{g a s, c h 4}\right) \\
& \rho_{T, 10}=k_{L} a\left(S_{c o 2}-K_{H, c o 2} p_{g a s, c o 2}\right)
\end{aligned}
$$

\subsubsection{Processos de inibição}

Sendo $p H=-\log _{10}\left(S_{\mathrm{H}+}\right)$, os diferentes fatores de inibição são modelados por:

$$
\begin{aligned}
& I_{5,6}=I_{p H, a a} \cdot I_{I N, \lim } \\
& I_{7}=I_{p H, a a} \cdot I_{I N, \lim } \cdot I_{h 2, f a} \\
& I_{8,9}=I_{p H, a a} \cdot I_{I N, \lim } \cdot I_{h 2, c 4} \\
& I_{10}=I_{p H, a a} \cdot I_{I N, \lim } \cdot I_{h 2, p r o} \\
& I_{11}=I_{p H, a c} \cdot I_{I N, \lim } \cdot I_{n h 3} \\
& I_{12}=I_{p H, h 2} \cdot I_{I N, \lim } \\
& I_{20}=I_{I N, \lim } \\
& I_{21}=I_{h 2, \text { laco }} \cdot I_{I N, \lim } \\
& I_{p H, a a}= \begin{cases}\exp \left[-3\left(\frac{p H-p H_{U L, a a}}{p H_{U L, a a}-p H_{L L, a a}}\right)^{2}\right] & \text { se } p H<p H_{U L, a a} \\
1 & \text { se } p H>p H_{U L, a a}\end{cases} \\
& I_{p H, a c}= \begin{cases}\exp \left[-3\left(\frac{p H-p H_{U L, a c}}{p H_{U L, a c}-p H_{L L, a c}}\right)^{2}\right] & \text { se } p H<p H_{U L, a c} \\
1 & \text { se } p H>p H_{U L, a c}\end{cases}
\end{aligned}
$$




$$
\begin{aligned}
& I_{p H, h 2}=\left\{\begin{array}{l}
\exp \left[-3\left(\frac{p H-p H_{U L, h 2}}{p H_{U L, h 2}-p H_{L L, h 2}}\right)^{2}\right] \text { se } p H<p H_{U L, h 2} \\
\text { se } p H>p H_{U L, h 2}
\end{array}\right. \\
& I_{1 N, \text { lim }}=\frac{1}{1+K_{S, I N} / S_{1 N}} \\
& I_{h 2, f a}=\frac{1}{1+S_{h 2} / K_{I, h 2, f a}} \\
& I_{h 2, c 4}=\frac{1}{1+S_{h 2} / K_{1, h 2, c 4}} \\
& I_{h 2, p r o}=\frac{1}{1+S_{h 2} / K_{I, h 2, p r o}} \\
& I_{n h 3}=\frac{1}{1+S_{n h 3} / K_{I, n h 3}} \\
& I_{h 2, \text { laco }}=\frac{1}{1+S_{h 2} / K_{I, h 2, \text { laco }}}
\end{aligned}
$$

A fim de reduzir instabilidades numéricas no processo de inibição por $\mathrm{pH}$, Rosen e Jeppsson (2006) adotaram aos processos de inibição funções diferentes daquelas usadas por Batstone et al. (2002), que são descontínuas. Tais autores adotaram a chamada inibição de Hill baseada na concentração de hidrogênio ao invés do pH (SIEGRIST et al., 2002).

$$
I_{p H}=\frac{K_{p H}^{n}}{S_{H^{+}}^{n}+K_{p H}^{n}} \quad, \text { sendo } \quad K_{p H}=10^{-\frac{p H_{L L}+p U_{U L}}{2}}
$$

Vale ressaltar que os valores para o expoente $n$ dependem dos valores $p H_{L L}$ e $p H_{U L}$. Em caso de alteração de tais limites, sugeriu-se adotar as expressões abaixo:

$$
n_{a a}=\frac{3.0}{p H_{U L, a a}-p H_{L L, a a}} \quad, \quad n_{a c}=\frac{3.0}{p H_{U L, a c}-p H_{L L, a c}} \quad, \quad n_{h 2}=\frac{3.0}{p H_{U L, h 2}-p H_{L L, h 2}}
$$

\subsubsection{Fase líquida}

As equações diferenciais da matéria solúvel, obtidas pelo balanço de massa para concentração de cada componente e incluindo a presença de lactato, são as seguintes:

$$
\begin{aligned}
& \frac{d S_{s u}}{d t}=\frac{q_{i n}}{V_{l i q}}\left(S_{s u, i n}-S_{s u}\right)+\rho_{2}+\left(1-f_{f a, l i}\right) \rho_{4}-\rho_{5} \\
& \frac{d S_{a a}}{d t}=\frac{q_{i n}}{V_{l i q}}\left(S_{a a, i n}-S_{a a}\right)+\rho_{3}-\rho_{6}
\end{aligned}
$$




$$
\begin{aligned}
& \frac{d S_{f a}}{d t}=\frac{q_{i n}}{V_{l i q}}\left(S_{f a, i n}-S_{f a}\right)+f_{f a, l i} \rho_{4}-\rho_{7} \\
& \frac{d S_{v a}}{d t}=\frac{q_{i n}}{V_{l i q}}\left(S_{v a, i n}-S_{v a}\right)+\left(1-Y_{a a}\right) f_{v a, a a} \rho_{6}-\rho_{8} \\
& \frac{d S_{s u}}{d t}=\frac{q_{i n}}{V_{l i q}}\left(S_{b u, i n}-S_{b u}\right)+\left(1-Y_{s u}\right) f_{b u, s u} \rho_{5}+\left(1-Y_{a a}\right) f_{b u, a a} \rho_{6}-\rho_{9} \\
& \frac{d S_{p r o}}{d t}=\frac{q_{\text {in }}}{V_{\text {liq }}}\left(S_{\text {pro, in }}-S_{\text {pro }}\right)+\left(1-Y_{\text {su }}\right) f_{\text {pro }, \text { su }} \rho_{5}+\left(1-Y_{a a}\right) f_{\text {pro, aa }} \rho_{6}+\left(1-Y_{c 4}\right) 0.54 \rho_{8} \\
& -\rho_{10}+\left(1-Y_{\text {lacf }}\right) 0,785_{\rho 20} \\
& \frac{d S_{a c}}{d t}=\frac{q_{i n}}{V_{l i q}}\left(S_{a c, \text { in }}-S_{a c}\right)+\left(1-Y_{s u}\right) f_{a c, s u} \rho_{5}+\left(1-Y_{a a}\right) f_{a c, a a} \rho_{6}+\left(1-Y_{f a}\right) 0.7 \rho_{7} \\
& +\left(1-Y_{c 4}\right) 0.31 \rho_{8}+\left(1-Y_{c 4}\right) 0.8 \rho_{9}+\left(1-Y_{p r o}\right) 0.57 \rho_{10}-\rho_{11}+\left(1-Y_{\text {lacf }}\right) 0,215_{\rho 20} \\
& +\left(1-Y_{\text {laco }}\right) 2 / 3_{\rho 21} \\
& \frac{d S_{h 2}}{d t}=\frac{q_{\text {in }}}{V_{l i q}}\left(S_{h 2, i n}-S_{h 2}\right)+\left(1-Y_{s u}\right) f_{h 2, s u} \rho_{5}+\left(1-Y_{a a}\right) f_{h 2, a a} \rho_{6}+\left(1-Y_{f a}\right) 0.3 \rho_{7}+ \\
& +\left(1-Y_{c 4}\right) 0.15 \rho_{8}+\left(1-Y_{c 4}\right) 0.2 \rho_{9}+\left(1-Y_{p r o}\right) 0.43 \rho_{10}-\rho_{12}-\rho_{T, 8}+\left(1-Y_{\text {laco }}\right) 1 / 3_{\rho 21} \\
& \frac{d S_{c h 4}}{d t}=\frac{q_{i n}}{V_{l i q}}\left(S_{c h 4, i n}-S_{c h 4}\right)+\left(1-Y_{a c}\right) \rho_{11}+\left(1-Y_{h 2}\right) \rho_{12}-\rho_{T, 9} \\
& \frac{d S_{I C}}{d t}=\frac{q_{i n}}{V_{l i q}}\left(S_{I C, i n}-S_{I C}\right)-\sum_{j=1}^{23}\left(\sum_{i=1-9,11-24,36-38} C_{i} v_{i, j} \rho_{j}\right)-\rho_{T, 10} \\
& \frac{d S_{I N}}{d t}=\frac{q_{i n}}{V_{l i q}}\left(S_{I N, i n}-S_{I N}\right)-Y_{s u} N_{b a c \rho 5}+\left(N_{a a}-Y_{a a} N_{b a c}\right) \rho_{6}-Y_{f a} N_{b a c} \rho_{7}-Y_{c 4} N_{b a c} \rho_{8}- \\
& Y_{c 4} N_{b a c} \rho_{9}-Y_{p r o} N_{b a c} \rho_{10}-Y_{a c} N_{b a c} \rho_{11}-Y_{h 2} N_{b a c} \rho_{12}-Y_{\text {lacf }} N_{b a c} \rho_{20}-Y_{\text {laco }} N_{b a c} \rho_{21} \\
& +\left(N_{b a c}-N_{x c}\right) \sum_{i=13-19,22-23} \rho_{i}+\left(N_{x c}-f_{x I, x c} N_{I}-f_{s I, x c} N_{I}-f_{p r, x c} N_{a a}\right) \rho_{1} \\
& \frac{d S_{I}}{d t}=\frac{q_{\text {in }}}{V_{l i q}}\left(S_{I, \text { in }}-S_{I}\right)+f_{s I, x c} \rho_{1}
\end{aligned}
$$

O somatório (com índice $j$ variando de 1 a 23) necessário à Eq. (70) é avaliado conforme:

$$
\sum_{j=1}^{23}\left(\sum_{i=1-9,11-24,36-38} C_{i} v_{i, j} \rho_{i}\right)=\sum_{k=1-12,20-21} \begin{gathered}
s_{k} \rho_{k}+s_{13}\left(\rho_{13}+\rho_{14}+\rho_{22}+\rho_{23}\right)
\end{gathered}
$$

sendo:

$$
s_{1}=-C_{x c}+f_{s I, x c} C_{s I}+f_{c h, x c} C_{c h}+f_{p r, x c} C_{p r}+f_{l i, x c} C_{l i}+f_{x I, x c} C_{x I}
$$




$$
\begin{aligned}
& s_{2}=-C_{c h}+C_{s u} \\
& s_{3}=-C_{p r}+C_{a a} \\
& s_{4}=-C_{l i}+\left(1-f_{f a, l i}\right) C_{s u}+f_{f a, l i} C_{f a} \\
& s_{5}=-C_{s u}+\left(1-Y_{s u}\right)\left(f_{b u, s u} C_{b u}+f_{p r o, s u} C_{p r o}+f_{a c, s u} C_{a c}\right)+Y_{s u} C_{b a c} \\
& s_{6}=-C_{a a}+\left(1-Y_{a a}\right)\left(f_{v a, a a} C_{v a}+f_{b u, a a} C_{b u}+f_{p r o, a a} C_{p r o}+f_{a c, a a} C_{a c}\right)+Y_{a a} C_{b a c} \\
& s_{7}=-C_{f a}+\left(1-Y_{f a}\right) 0.7 C_{a c}+Y_{f a} C_{b a c} \\
& s_{8}=-C_{v a}+\left(1-Y_{c 4}\right) 0.54 C_{p r o}+\left(1-Y_{c 4}\right) 0.31 C_{a c}+Y_{c 4} C_{b a c} \\
& s_{9}=-C_{b u}+\left(1-Y_{c 4}\right) 0.8 C_{a c}+Y_{c 4} C_{b a c} \\
& s_{10}=-C_{p r o}+\left(1-Y_{p r o}\right) 0.57 C_{a c}+Y_{p r o} C_{b a c} \\
& s_{11}=-C_{a c}+\left(1-Y_{a c}\right) C_{c h 4}+Y_{a c} C_{b a c} \\
& s_{12}=\left(1-Y_{h 2}\right) C_{c h 4}+Y_{h 2} C_{b a c} \\
& s_{13}=-C_{b a c}+C_{x c} \\
& s_{20}=-C_{l a c}+\left(1-Y_{l a c f}\right) 0,785 C_{p r o}+\left(1-Y_{l a c f}\right) 0,215 C_{a c}+Y_{l a c f} C_{b a c} \\
& s_{21}=-C_{l a c}+\left(1-Y_{l a c o}\right) 2 / 3 C_{a c}+Y_{l a c o} C_{b a c}
\end{aligned}
$$

As equações diferenciais da matéria particulada são as seguintes:

$$
\begin{gathered}
\frac{d X_{c}}{d t}=\frac{q_{i n}}{V_{l i q}}\left(X_{c, i n}-X_{c}\right)-\rho_{1}+\sum_{i=13-19,22-23} \rho_{i} \\
\frac{d X_{c h}}{d t}=\frac{q_{i n}}{V_{l i q}}\left(X_{c h, i n}-X_{c h}\right)+f_{c h, x c} \rho_{1}-\rho_{2} \\
\frac{d X_{p r}}{d t}=\frac{q_{i n}}{V_{l i q}}\left(X_{p r, i n}-X_{p r}\right)+f_{p r, x c} \rho_{1}-\rho_{3} \\
\frac{d X_{l i}}{d t}=\frac{q_{i n}}{V_{l i q}}\left(X_{l i, i n}-X_{l i}\right)+f_{l i, x c} \rho_{1}-\rho_{4} \\
\frac{d X_{s u}}{d t}=\frac{q_{i n}}{V_{l i q}}\left(X_{s u, i n}-X_{s u}\right)+Y_{s u} \rho_{5}-\rho_{13} \\
\frac{d X_{a a}}{d t}=\frac{q_{i n}}{V_{l i q}}\left(X_{a a, i n}-X_{a a}\right)+Y_{a a} \rho_{6}-\rho_{14}
\end{gathered}
$$




$$
\begin{aligned}
& \frac{d X_{f a}}{d t}=\frac{q_{i n}}{V_{l i q}}\left(X_{f a, i n}-X_{f a}\right)+Y_{f a} \rho_{7}-\rho_{15} \\
& \frac{d X_{c 4}}{d t}=\frac{q_{i n}}{V_{l i q}}\left(X_{c 4, i n}-X_{c 4}\right)+Y_{c 4} \rho_{8}+Y_{c 4} \rho_{9}-\rho_{16} \\
& \frac{d X_{p r o}}{d t}=\frac{q_{i n}}{V_{l i q}}\left(X_{p r o, i n}-X_{p r o}\right)+Y_{p r o} \rho_{10}-\rho_{17} \\
& \frac{d X_{a c}}{d t}=\frac{q_{i n}}{V_{l i q}}\left(X_{a c, i n}-X_{a c}\right)+Y_{a c} \rho_{11}-\rho_{18} \\
& \frac{d X_{h 2}}{d t}=\frac{q_{i n}}{V_{l i q}}\left(X_{h 2, i n}-X_{h 2}\right)+Y_{h 2} \rho_{12}-\rho_{19} \\
& \frac{d X_{I}}{d t}=\frac{q_{i n}}{V_{l i q}}\left(X_{I, i n}-X_{I}\right)+f_{x I, x c} \rho_{1}
\end{aligned}
$$

Também foram incluídas as adaptações feitas por Danielsson (2014) na inclusão do processo de precipitação de carbonato de cálcio (transferência sólido-líquido), a saber:

$$
\begin{aligned}
& \frac{d X_{\text {lac }}}{d t}=\frac{q_{\text {in }}}{V_{\text {liq }}}\left(X_{\text {lac }, \text { in }}-X_{\text {lac }}\right) \rho_{20}-\rho_{21} \\
& \frac{d X_{\text {lac }} f}{d t}=\frac{q_{\text {in }}}{V_{\text {liq }}}\left(X_{\text {lacf }, \text { in }}-X_{\text {lacf }}\right)+Y_{\text {lacf }} \rho_{20}-\rho_{22} \\
& \frac{d X_{\text {laco }}}{d t}=\frac{q_{\text {in }}}{V_{\text {liq }}}\left(X_{\text {laco ,in }}-X_{\text {laco }}\right)+Y_{\text {laco }} \rho_{22}-\rho_{23} \\
& \frac{d S_{c a}}{d t}=\frac{q_{\text {in }}}{V_{\text {liq }}}\left(S_{\text {ca,in }}-S_{\text {ca }}\right)+\rho_{P 24}
\end{aligned}
$$

Adicionalmente, foi considerada a equação abaixo para DQO total:

$$
\begin{aligned}
& \frac{d S_{C O D}}{d t}=\frac{q_{\text {in }}}{V_{l i q}}\left(S_{C O D, i n}-S_{C O D}\right)+\frac{d S_{s u}}{d t}+\frac{d S_{v a}}{d t}+\frac{d S_{b u}}{d t}+\frac{d S_{p r o}}{d t}+\frac{d S_{a c}}{d t}+\frac{d S_{h 2}}{d t}+ \\
& \frac{d S_{c h 4}}{d t}+\frac{d S_{v a^{-}}}{d t}+\frac{d S_{b u^{-}}}{d t}+\frac{d S_{p r o^{-}}}{d t}+\frac{d S_{a c^{-}}}{d t}
\end{aligned}
$$

Abaixo são apresentadas as equações de cátions e ânions, seguidas das equações de estado de íons:

$$
\frac{d S_{c a t^{+}}}{d t}=\frac{q_{\text {in }}}{V_{l i q}}\left(S_{c a t^{+}, i n}-S_{c a t^{+}}\right)
$$




$$
\begin{aligned}
& \frac{d S_{a n^{-}}}{d t}=\frac{q_{i n}}{V_{l i q}}\left(S_{a n^{-}, i n}-S_{a n^{-}}\right) \\
& \frac{d S_{v a^{-}}}{d t}=-\rho_{A, 4} \\
& \frac{d S_{b u^{-}}}{d t}=-\rho_{A, 5} \\
& \frac{d S_{p r 0^{-}}}{d t}=-\rho_{A, 6} \\
& \frac{d S_{a c^{-}}}{d t}=-\rho_{A, 7} \\
& \frac{d S_{h c o 3^{-}}}{d t}=-\rho_{A, 10} \\
& \frac{d S_{n h 3}}{d t}=-\rho_{A, 11}
\end{aligned}
$$

Equação algébrica a seguir é usada para avaliar o pH:

$$
S_{H^{+}}=-\frac{\Theta}{2}+\frac{1}{2} \sqrt{\Theta^{2}+4 K_{W}}
$$

em que:

$$
\begin{aligned}
& \Theta=S_{c a t^{+}}+S_{n h 4^{+}}-S_{h c o 3^{-}}-\frac{S_{a c^{-}}}{64}-\frac{S_{p r 0^{-}}}{112}-\frac{S_{b u^{-}}}{160}-\frac{S_{v a^{-}}}{208}-S_{a n^{-}}+S_{C a^{+}} \\
& \text {com } S_{n h 4^{+}}=S_{I N}-S_{n h 3} \text { e } S_{c o 2}=S_{I C}-S_{h c o 3^{-}}
\end{aligned}
$$

\subsubsection{Fase gasosa}

As equações diferenciais da matéria solúvel são as seguintes:

$$
\begin{aligned}
& \frac{d S_{g a s, h 2}}{d t}=-\frac{S_{g a s, h 2} q_{g a s}}{V_{g a s}}+\rho_{T, 8} \frac{V_{l i q}}{V_{g a s}} \\
& \frac{d S_{g a s, c h 4}}{d t}=-\frac{S_{g a s, c h 4} q_{g a s}}{V_{g a s}}+\rho_{T, 9} \frac{V_{l i q}}{V_{g a s}} \\
& \frac{d S_{g a s, c o 2}}{d t}=-\frac{S_{g a s, c o 2} q_{g a s}}{V_{g a s}}+\rho_{T, 10} \frac{V_{l i q}}{V_{g a s}}
\end{aligned}
$$

Tais equações requerem as seguintes equações algébricas para as pressões parciais:

$$
p_{g a s, h 2}=S_{g a s, h 2} \frac{R T_{o p}}{16} \quad, \quad p_{g a s, c h 4}=S_{g a s, c h 4} \frac{R T_{o p}}{64} \quad, \quad p_{g a s, c o 2}=S_{g a s, c o 2} R T_{o p}
$$

e para a vazão volumétrica de gás: 


$$
q_{\text {gas }}=\frac{R T_{o p}}{P_{a t m}-p_{g a s, H_{2} O}} V_{l i q}\left(\frac{\rho_{T, 8}}{16}+\frac{\rho_{T, 9}}{64}+\rho_{T, 10}\right)
$$

Rosen e Jeppsson (2006) utilizaram uma equação alternativa para avaliar a vazão de gás, assumindo uma pressão maior na fase gasosa na parte superior do reator. A meta foi evitar problemas numéricos reportados ao usar a equação 120. Tal equação alternativa é apresentada a seguir:

$$
q_{\text {gas }}=k\left(P_{\text {gas }}-P_{a t m}\right), \text { com } P_{\text {gas }}=p_{\text {gas }, h 2}+p_{\text {gas }, c h 4}+p_{\text {gas }, c o 2}+p_{\text {gas }, h 2 O}
$$

A expressão abaixo pode ser usada para compensar a diferença de pressão e obtenção da vazão na pressão atmosférica:

$$
q_{g a s}=k_{p}\left(P_{g a s}-P_{a t m}\right) \frac{P_{g a s}}{P_{a t m}}
$$




\section{METODOLOGIA}

No primeiro ano de pesquisa deste projeto, objetivou-se aprofundar os conhecimentos sobre o modelo ADM1 e sobre a linguagem de programação Python. O segundo ano foi dedicado à implementação do simulador e aos ajustes (modelo e simulador) que se fizeram necessários ao longo do processo.

Um desafio para simular numericamente o modelo ADM1 refere-se à variabilidade das cinéticas químicas (de segundos a meses), de modo que parte do sistema reage muito rapidamente enquanto que outra evolui muito lentamente. Portanto, o solver ("solucionador") deve ser capaz de lidar com tal dificuldade numérica.

Rosen e Jeppsson (2006) recorreram ao MATLAB/SIMULINK; alternativamente, o presente trabalho usou o ambiente Python de programação. Com as alterações sugeridas por Rosen e Jeppsson (2006) e Danielsson (2014), o modelo ADM1 foi programado utilizando o ambiente integrado Spyder e o software Anaconda, de acordo com o algoritmo descrito na Figura 2.

Figura 2 - Algoritmo de programação do modelo ADM1

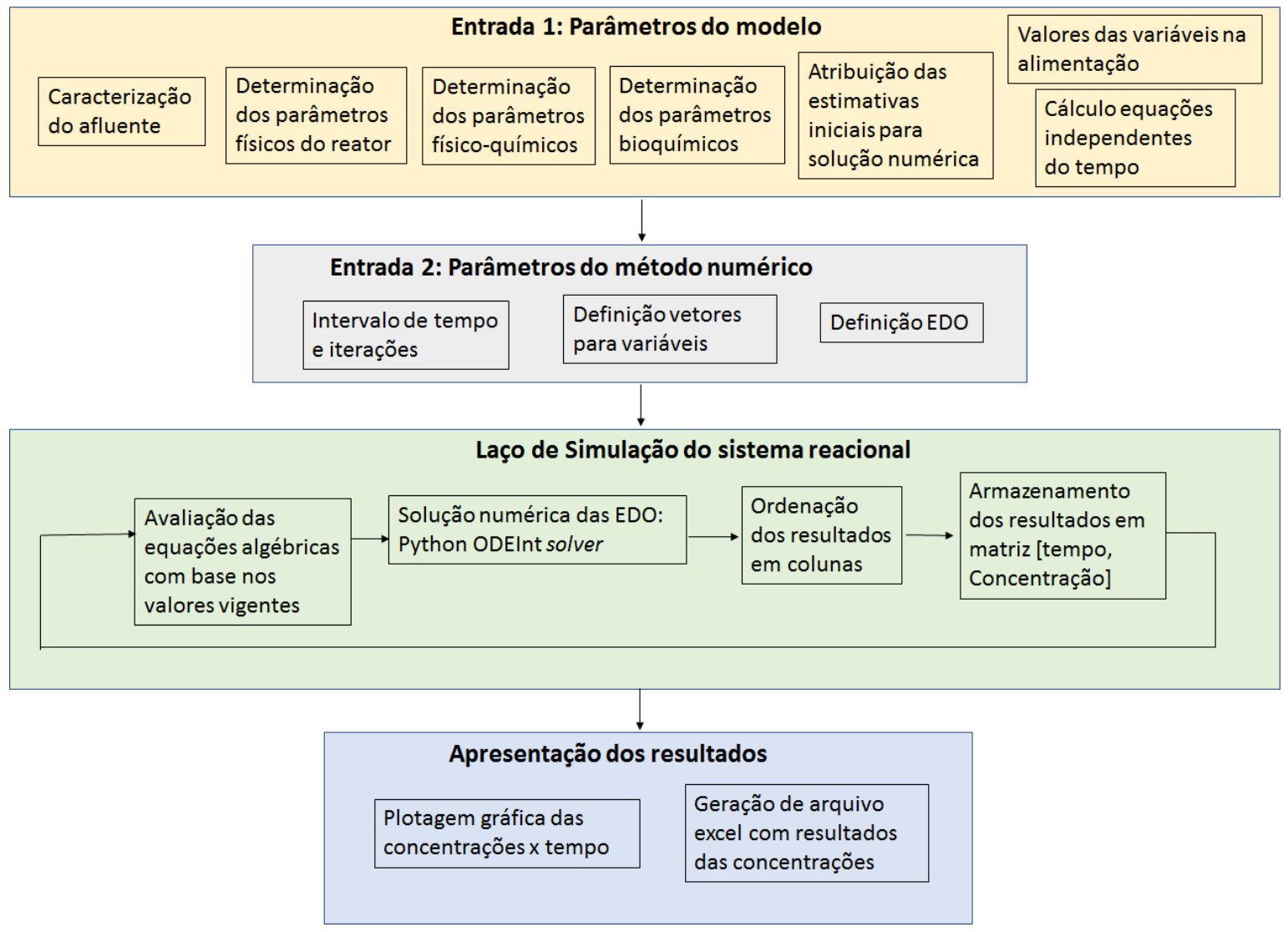

Fonte: Própria autoria. 
O algoritmo da Figura 2 mostra o fluxo das informações necessárias para a solução numérica do problema de valor inicial. Primeiramente, é feita a caracterização do afluente, definindo-se os parâmetros do modelo e condições operacionais. Na sequência são lidas as condições iniciais, sendo $S_{\text {var,in }}^{n=0}$ os valores na alimentação para cada espécie solúvel "var", $X_{\text {var,in }}^{n=0}$ para as espécies particuladas e $S_{v a r}^{n=0}$ e $X_{\text {var }}^{n=0}$ a estimativa inicial para solução das variáveis de estado dinâmico, conforme ilustração da Figura 3.

Figura 3 - Esquema ilustrativo das concentrações e nomenclaturas definidas no reator

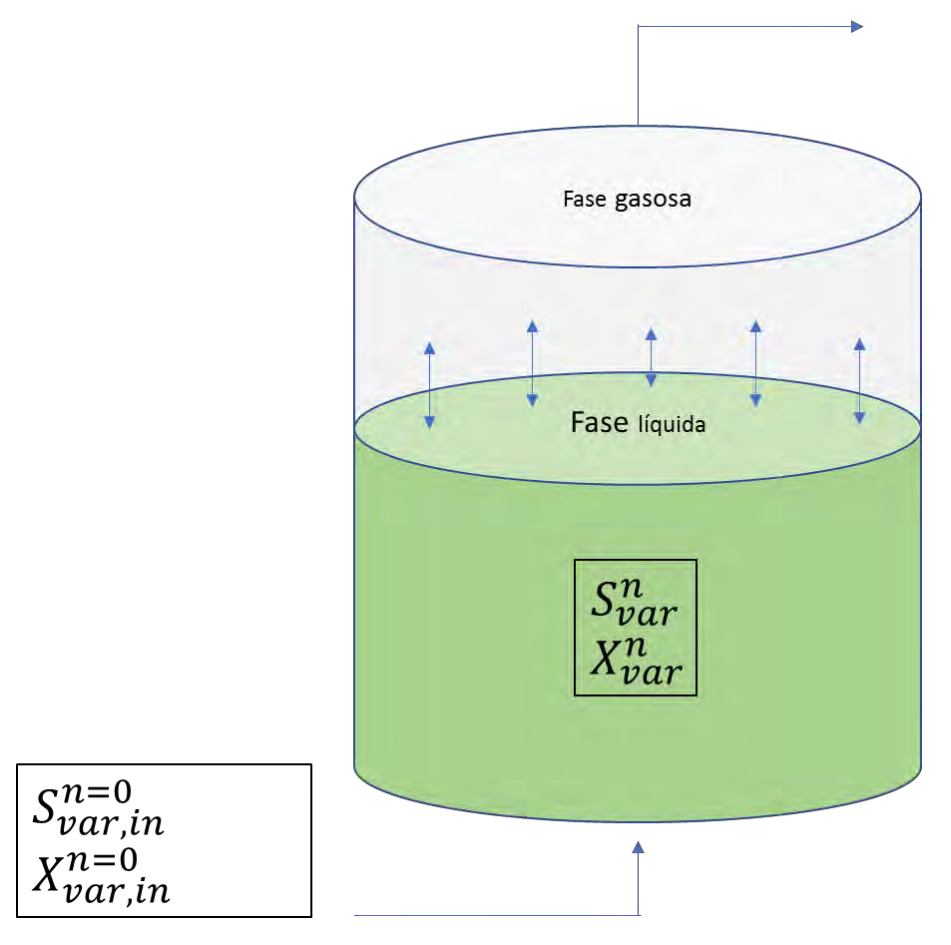

Fonte: Própria autoria.

As equações diferenciais ordinárias (EDOs) foram definidas de forma agrupada dentro de uma única função computacional. É executado então o solucionador das EDOs para todo o conjunto de equações, enquanto não se atinge o instante final de processo simulado $\left(t<t_{\text {final }}\right)$. O laço se repete para o número de iteração $n$ definido para cada intervalo de tempo $\Delta t(t[i+1]$ $-t[i])$, conforme a Tabela 2.

Tabela 2 - Intervalo de iterações

\begin{tabular}{cccccc}
\hline$n$ (número da iteração) & 0 & 1 & 2 & $\ldots$ & $N$ \\
\hline$t$ (tempo de processo simulado) & 0 & $\Delta t$ & $2 \Delta t$ & $\ldots$ & $t_{\text {final }}$ \\
\hline
\end{tabular}

Fonte: Própria autoria. 
No presente trabalho foram simulados diferentes cenários de tratamento anaeróbio de efluentes agroindustriais, descritos a seguir. Nos dois primeiros cenários (simulações 1 e 2) foi desconsiderada a etapa de hidrólise e foram consideradas apenas as etapas envolvidas a partir da degradação de açúcares (adotada glicose como base ADM1), conforme discutido em capítulo anterior.

- Simulação 1: Na primeira simulação foi implementado o modelo ADM1 utilizando os parâmetros descritos nas Tabela 3 a 15, baseados nas simulações dos trabalhos de Danielsson (2014) e Rosén e Jeppsson (2006), porém excluindo-se a etapa de hidrólise e considerando apenas a degradação de açúcares (glicose) para simplificar o modelo e facilitar a avaliação da aplicabilidade e questões numéricas. Estes parâmetros foram utilizados para simular um processo de digestão com 50 dias de duração (Simulação 1-A), assim como adotado por Danielsson (2014). Mediante os resultados desta primeira simulação (1-A) foi realizada outra simulação por um período de processo de digestão de 5 dias (Simulação 1-B), para avaliar melhor os perfis de concentração de algumas espécies que tiveram resultado comprometido no período mais longo, de 50 dias. Adicionalmente, os mesmos parâmetros foram empregados na Simulação 1-C, realizada com alteração nos valores da estimativa inicial, por um tempo de processo de 50 dias;

- Simulação 2: Na segunda simulação foi feita implementação do modelo ADM1 utilizando os parâmetros descritos nas Tabelas 3 a 15, mas alguns deles com valores alterados com base em pesquisas próprias do grupo AGROENERBIO. Esta simulação considerou um período de digestão anaeróbia de 50 dias, para avaliar o comportamento do simulador com alterações nos parâmetros de processo e do modelo;

- Simulação 3: Nesta simulação foi feita implementação do modelo ADM1 completo, com todos os parâmetros listados nas Tabela 3 a 15. Além destes, as Tabelas 16 (a)-(i) listam demais parâmetros necessários para a simulação do modelo ADM1 completo. Estes parâmetros foram utilizados para simular um processo de digestão de 50 dias, para fins de comparação com os resultados obtidos por Danielsson (2014) e Rosen e Jeppsson (2006) e para aproximar o simulador do processo de digestão anaeróbia da vinhaça, para futuras adequações às especificidades dos substratos da vinhaça. 
Tabela 3 - Parâmetros da cinética biológica: taxas de crescimento máximo

\begin{tabular}{|c|c|c|c|}
\hline \multicolumn{2}{|c|}{$\begin{array}{l}\text { Constante de cinética biológica relacionada à velocidade máxima de } \\
\text { crescimento bacteriano (para cada grupo de bactérias) }\left[\mathrm{d}^{-1}\right]\end{array}$} & \multirow{2}{*}{$\begin{array}{c}\text { Simulação } \\
\mathbf{1}\end{array}$} & \multirow{2}{*}{$\begin{array}{c}\text { Simulação } \\
\mathbf{2} \\
1,805\end{array}$} \\
\hline$k_{\mathrm{m}, \mathrm{su}}$ & Biomassa consumidora de açúcar (glicose) & & \\
\hline$k_{\mathrm{m}, \mathrm{c} 4}$ & Biomassa consumidora de valerato e butirato & 20 & 20 \\
\hline$k_{\mathrm{m}, \text { pro }}$ & Biomassa consumidora de propionato & 13 & 13 \\
\hline$k_{\mathrm{m}, \mathrm{ac}}$ & Biomassa consumidora de acetato & 8 & 8 \\
\hline$k_{\mathrm{m}, \mathrm{h} 2}$ & Biomassa consumidora de gás hidrogênio & 35 & 35 \\
\hline$k_{\mathrm{m}, \mathrm{lac}, \mathrm{f}}{ }^{*}$ & Biomassa consumidora de lactato & 16 & 16 \\
\hline$k_{\mathrm{m}, \mathrm{lac}, \mathrm{o}}{ }^{*}$ & Biomassa consumidora de lactato & 16 & 16 \\
\hline
\end{tabular}

* Parâmetros extras - modelo ADM1. Fonte: Própria autoria.

Tabela 4 - Parâmetros adimensionais de cinética biológica: taxas de produção de biomassa

\begin{tabular}{llcc}
\hline \multicolumn{2}{c}{ Taxa de produção de biomassa (para cada grupo de bactérias) } & Simulação 1 & Simulação 2 \\
\hline$Y_{\mathrm{su}}$ & Biomassa consumidora de açúcar & 0,1 & 0,1 \\
$Y_{\mathrm{c} 4}$ & Biomassa consumidora de valerato e butirato & 0,06 & 0,06 \\
$Y_{\mathrm{pro}}$ & Biomassa consumidora de propionato & 0,04 & 0,04 \\
$Y_{\mathrm{ac}}$ & Biomassa consumidora de acetato & 0,05 & 0,05 \\
$Y_{\mathrm{h} 2}$ & Biomassa consumidora de gás hidrogênio & 0,06 & 0,06 \\
$Y_{\text {lac,f }}{ }^{*}$ & Biomassa consumidora lactato & 0,055 & 0,055 \\
$Y_{\text {lac,o }}$ & Biomassa consumidora lactato & 0,055 & 0,055 \\
\hline
\end{tabular}

* Parâmetros extras - modelo ADM1. Fonte: Própria autoria.

Tabela 5 - Parâmetros de cinética biológica: constantes de Monod

\begin{tabular}{|c|c|c|c|}
\hline \multicolumn{2}{|c|}{ 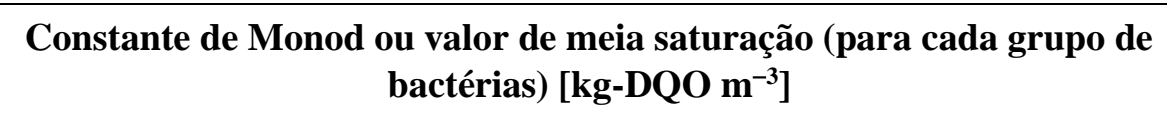 } & \multirow{2}{*}{$\begin{array}{c}\begin{array}{c}\text { Simulação } \\
\mathbf{1}\end{array} \\
0,5\end{array}$} & \multirow{2}{*}{$\begin{array}{c}\text { Simulação } \\
\mathbf{2} \\
0,0563\end{array}$} \\
\hline$K_{\mathrm{S}, \mathrm{su}}$ & Biomassa consumidora de açúcar & & \\
\hline$K_{\mathrm{S}, \mathrm{c} 4}$ & Biomassa consumidora de valerato e butirato & 0,2 & 0,2 \\
\hline$K_{\mathrm{S} \text {,pro }}$ & Biomassa consumidora de propionato & 0,1 & 0,1 \\
\hline$K_{\mathrm{S}, \mathrm{ac}}$ & Biomassa consumidora de acetato & 0,15 & 0,15 \\
\hline$K_{\mathrm{S}, \mathrm{h} 2}$ & Biomassa consumidora de gás hidrogênio & $7,00 \times 10^{-6}$ & $7,00 \times 10^{-6}$ \\
\hline$K_{\mathrm{S}, \mathrm{IN}}$ & Biomassa consumidora de inertes & $1,00 \times 10^{-4}$ & $1,00 \times 10^{-4}$ \\
\hline$K_{\mathrm{S}, \mathrm{lac}, \mathrm{f}}{ }^{*}$ & Biomassa consumidora de lactato (processo de fermentação) & 3,5169 & 3,5169 \\
\hline$K_{\mathrm{S}, \mathrm{lac}, \mathrm{o}}{ }^{*}$ & Biomassa consumidora de lactato (processo de oxidação) & 0,6432 & 0,6432 \\
\hline$K_{\mathrm{S}, \mathrm{p}, \mathrm{caco3}}{ }^{*}$ & Biomassa consumidora de carbonato de cálcio (precipitação) & $1,92 \times 10^{-5}$ & $1,92 \times 10^{-5}$ \\
\hline
\end{tabular}

* Parâmetros extras - modelo ADM1. Fonte: Própria autoria. 
Tabela 6 - Parâmetros de cinética biológica: taxas de decaimento (consumo)

\begin{tabular}{|c|c|c|c|}
\hline \multicolumn{2}{|r|}{ Taxa de decaimento (para cada grupo de bactérias) $\left[d^{-1}\right]$} & \multirow{2}{*}{$\frac{\text { Simulação 1 }}{0,02}$} & \multirow{2}{*}{$\frac{\text { Simulação 2 }}{0,02}$} \\
\hline$k_{\mathrm{dec}, \mathrm{Xsu}}$ & Biomassa consumidora de açúcar & & \\
\hline$k_{\mathrm{dec}, \mathrm{Xc} 4}$ & Biomassa consumidora de valerato e butirato & 0,02 & 0,02 \\
\hline$k_{\mathrm{dec}, \mathrm{Xpro}}$ & Biomassa consumidora de propionato & 0,02 & 0,02 \\
\hline$k_{\mathrm{dec}, \mathrm{Xac}}$ & Biomassa consumidora de acetato & 0,02 & 0,02 \\
\hline$k_{\mathrm{dec}, \mathrm{Xh} 2}$ & Biomassa consumidora de gás hidrogênio & 0,02 & 0,02 \\
\hline$k_{\mathrm{dec}, \mathrm{Xlac}, \mathrm{f}}{ }^{*}$ & Biomassa consumidora de lactato (fermentação) & 0,02 & 0,02 \\
\hline$k_{\text {dec,Xlac,o }}{ }^{*}$ & Biomassa consumidora de lactato (oxidação) & 0,02 & 0,02 \\
\hline$K_{\mathrm{r}, \mathrm{caco} 3}{ }^{*}$ & Biomassa consumidora de carbonato de cálcio (precipitação) & 1477,44 & 1477,44 \\
\hline
\end{tabular}

* Parâmetros extras - modelo ADM1. Fonte: Própria autoria.

Tabela 7 - Parâmetros para a inibição por pH

\begin{tabular}{llcc}
\hline & Limites de pH definidos para a inibição [adimensionais] & Simulação 1 & Simulação 2 \\
\hline$p H_{\mathrm{UL}, \mathrm{ac}}$ & Limite superior da inibição por $\mathrm{pH}$ no consumo de acetato & 7 & 7 \\
$p H_{\mathrm{LL}, \mathrm{ac}}$ & Limite inferior da inibição por $\mathrm{pH}$ no consumo de acetato & 6 & 6 \\
$p H_{\mathrm{UL}, \mathrm{h} 2}$ & Limite superior da inibição por $\mathrm{pH}$ no consumo de hidrogênio & 6 & 6 \\
$p H_{\mathrm{LL}, \mathrm{h} 2}$ & Limite inferior da inibição por $\mathrm{pH}$ no consumo de hidrogênio & 5 & 5 \\
$p H_{\mathrm{UL}, \mathrm{aa}}$ & Limite superior da inibição por $\mathrm{pH}$ no consumo de aminoácidos & 5,5 & 5,5 \\
$p H_{\mathrm{LL}, \mathrm{aa}}$ & Limite inferior da inibição por $\mathrm{pH}$ no consumo de aminoácidos & 4 & 4 \\
\hline
\end{tabular}

Fonte: Própria autoria.

Tabela 8 - Parâmetros para inibição por $\mathrm{H}_{2}$

\begin{tabular}{|c|c|c|c|}
\hline \multicolumn{2}{|c|}{$\begin{array}{c}\text { Concentrações de hidrogênio que inibem em } 50 \% \text { certos grupos de } \\
\text { bactérias }\left[\mathrm{kg}-\mathrm{DQO} \mathrm{m}^{-3}\right]\end{array}$} & \multirow{2}{*}{$\begin{array}{c}\text { Simulação } \\
\mathbf{1}\end{array}$} & \multirow{2}{*}{$\begin{array}{c}\text { Simulação } \\
\mathbf{2} \\
1,00 \times 10^{-5}\end{array}$} \\
\hline$K_{\mathrm{I}, \mathrm{h} 2, \mathrm{c} 4}$ & Bactérias que consomem valerato e butirato & & \\
\hline$K_{\mathrm{I}, \mathrm{h} 2 \text {,pro }}$ & Bactérias que consomem propionato & $3,50 \times 10^{-6}$ & $3,50 \times 10^{-6}$ \\
\hline$K_{\mathrm{I}, \mathrm{h} 2, \mathrm{lac}, \mathrm{o}}{ }^{*}$ & Bactérias que consomem lactato & $1,40 \times 10^{-4}$ & $1,40 \times 10^{-4}$ \\
\hline
\end{tabular}

* Parâmetros extras - modelo ADM1. Fonte: Própria autoria.

Tabela 9 - Parâmetro para inibição por $\mathrm{NH}_{3}$

\begin{tabular}{lccc}
\hline \multicolumn{2}{r}{$\begin{array}{c}\text { Concentração de amônia livre que inibe em } \\
\text { arqueias }\left[\mathbf{k g}-\mathrm{DQO} \mathbf{~ m}^{-\mathbf{3}}\right]\end{array}$} & $\begin{array}{c}\text { Simulação um grupo de } \\
\mathbf{1}\end{array}$ & $\begin{array}{c}\text { Simulação } \\
\mathbf{2}\end{array}$ \\
\hline$K_{\mathrm{I}, \mathrm{nh} 3}$ & Arqueias que consomem acetato para a metanogênese & 0,0018 & 0,0018 \\
\hline
\end{tabular}

Fonte: Própria autoria. 
Tabela 10 - Parâmetro da quantidade de carbono nas variáveis de estado do modelo

\begin{tabular}{llcc}
\hline & Quantidade de carbono nos componentes[kmol kg-DQO-1] & Simulação 1 & Simulação 2 \\
\hline$C_{\mathrm{xc}}$ & No material particulado & 0,02786 & 0,02786 \\
$C_{\mathrm{su}}$ & Em monossacarídeos & 0,0313 & 0,0313 \\
$C_{\mathrm{bu}}$ & No butirato & 0,025 & 0,025 \\
$C_{\mathrm{pr}}$ & No propionato & 0,0268 & 0,0268 \\
$C_{\mathrm{ac}}$ & No acetato & 0,0313 & 0,0313 \\
$C_{\mathrm{bac}}$ & Nos grupos de bactérias & 0,0313 & 0,0313 \\
$C_{\mathrm{va}}$ & No valerato & 0,024 & 0,024 \\
$C_{\mathrm{ch} 4}$ & No metano & 0,0156 & 0,0156 \\
$C_{\mathrm{lac}}{ }^{*}$ & No lactato & 0,0313 & 0,0313 \\
\hline
\end{tabular}

* Parâmetro extra - modelo ADM1. Fonte: Própria autoria.

Tabela 11 - Parâmetro da quantidade de nitrogênio nas variáveis de estado do modelo

\begin{tabular}{llcc}
\hline \multicolumn{2}{c}{ Quantidade de nitrogênio nos componentes[kmol kg-DQO-1] } & Simulação 1 & Simulação 2 \\
\hline$N_{\mathrm{xc}}$ & No material particulado & 0,002685714 & 0,00268571 \\
$N_{\text {bac }}$ & Nos grupos de bactérias & 0,005714286 & 0,00571429 \\
\hline
\end{tabular}

Fonte: Própria autoria.

Tabela 12 - Parâmetros estequiométricos: taxas de formação em reações bioquímicas

\begin{tabular}{llcc}
\hline & Parâmetros estequiométricos [adimensionais] & Simulação 1 & Simulação 2 \\
\hline$f_{\mathrm{h} 2, \mathrm{su}}$ & Formação de gás hidrogênio a partir de monossacarídeos & 0,19 & 0,19 \\
$f_{\mathrm{bu}, \mathrm{su}}$ & Formação de butirato a partir de monossacarídeos & 0,13 & 0,13 \\
$f_{\text {pro,su }}$ & Formação de propionato a partir de monossacarídeos & 0,27 & 0,27 \\
$f_{\mathrm{ac}, \mathrm{su}}$ & Formação de acetato a partir de monossacarídeos & 0,41 & 0,41 \\
\hline
\end{tabular}

Fonte: Própria autoria.

Tabela 13 - Constantes de Henry para os gases

\begin{tabular}{llcc}
\hline & Constante de Henry para os gases[M bar $\left.{ }^{-1}\right]$ & Simulação 1 & Simulação 2 \\
\hline$K_{\mathrm{H}, \mathrm{ch} 4}$ & Constante de Henry para o gás metano & 0,001161903 & 0,0011619 \\
$K_{\mathrm{H}, \mathrm{co} 2}$ & Constante de Henry para o gás carbônico & 0,027146693 & 0,02714669 \\
$K_{\mathrm{H}, \mathrm{h} 2}$ & Constante de Henry para o gás hidrogênio & 0,000738465 & 0,00073847 \\
\hline
\end{tabular}

Fonte: Própria autoria. 
Tabela 14 - Parâmetros para os equilíbrios químicos

\begin{tabular}{llcc}
\hline & Coeficiente de equilíbrio ácido-base $\left[\mathbf{k m o l ~ m}^{-3}\right]$ & Simulação 1 & Simulação 2 \\
\hline$K_{\mathrm{a}, \mathrm{ac}}$ & $\mathrm{HAc} / \mathrm{Ac}^{-}$ & $1,74 \times 10^{-5}$ & $1,74 \times 10^{-5}$ \\
$K_{\mathrm{a}, \mathrm{bu}}$ & $\mathrm{HBu} / \mathrm{Bu}^{-}$ & $1,51 \times 10^{-5}$ & $1,51 \times 10^{-5}$ \\
$K_{\mathrm{a}, \mathrm{co} 2}$ & $\mathrm{CO}_{2} / \mathrm{HCO}_{3}^{-}$ & $4,94 \times 10^{-7}$ & $4,94 \times 10^{-7}$ \\
$K_{\mathrm{w}}$ & $\mathrm{H}_{2} \mathrm{O} / \mathrm{OH}^{-} \mathrm{e} \mathrm{H}^{+}$ & $2,07877 \times 10^{-14}$ & $2,07877 \times 10^{-14}$ \\
$K_{\mathrm{a}, \mathrm{pro}}$ & $\mathrm{HPro}^{-} \mathrm{Pro}^{-}$ & $1,32 \times 10^{-5}$ & $1,32 \times 10^{-5}$ \\
$K_{\mathrm{a}, \mathrm{va}}$ & $\mathrm{HVa} / \mathrm{Va}^{-}$ & $1,38 \times 10^{-5}$ & $1,38 \times 10^{-5}$ \\
$K_{\mathrm{a}, \mathrm{IN}}$ & $\mathrm{HIN} / \mathrm{IN}^{-}$ & $1,11 \times 10^{-9}$ & $1,11 \times 10^{-9}$ \\
$k_{\mathrm{A}, \mathrm{Bva}}$ & Parâmetro para valerato & $1.0 \times 10^{10}$ & $1.0 \times 10^{10}$ \\
$k_{\mathrm{A}, \mathrm{Bbu}}$ & Parâmetro para butirato & $1.0 \times 10^{10}$ & $1.0 \times 10^{10}$ \\
$k_{\mathrm{A}, \mathrm{Bpro}}$ & Parâmetro para propionato & $1.0 \times 10^{10}$ & $1.0 \times 10^{10}$ \\
$k_{\mathrm{A}, \mathrm{Bac}}$ & Parâmetro para acetato & $1.0 \times 10^{10}$ & $1.0 \times 10^{10}$ \\
$k_{\mathrm{A}, \mathrm{Bco} 2}$ & Parâmetro para carbono inorgânico & $1.0 \times 10^{10}$ & $1.0 \times 10^{10}$ \\
$k_{\mathrm{A}, \mathrm{BIN}}$ & Parâmetro para nitrogênio inorgânico & $1.0 \times 10^{10}$ & $1.0 \times 10^{10}$ \\
\hline
\end{tabular}

Fonte: Própria autoria.

Tabela 15 - Parâmetros do reator

\begin{tabular}{|c|c|c|c|}
\hline & Parâmetros do reator ou de operação & $\begin{array}{c}\text { Simulação 1 } \\
\text { (base ADM1) }\end{array}$ & $\begin{array}{c}\text { Simulação } 2 \\
\text { (Pesquisas próprias } \\
\text { do grupo } \\
\text { AGROENERBIO) }\end{array}$ \\
\hline$q_{\text {in }}$ & Vazão volumétrica de efluente $\left[\mathrm{m}^{3} \mathrm{~d}^{-1}\right]$ & 170 & 0 \\
\hline$V_{\text {liq }}$ & Volume de líquido no reator $\left[\mathrm{m}^{3}\right]$ & 3400 & 0,005 \\
\hline$V_{\text {gas }}$ & Volume ocupado pelos gases $\left[\mathrm{m}^{3}\right]$ & 300 & 0,0025 \\
\hline$k_{\mathrm{La}}$ & Coeficiente de transferência gás-líquido ${ }^{*}\left[\mathrm{~d}^{-1}\right]$ & 200 & 200 \\
\hline$T$ & Temperatura $[\mathrm{K}]$ & 308,15 & 308,15 \\
\hline$T_{\text {base }}$ & Temperatura $[\mathrm{K}]$ & 298,15 & 298,15 \\
\hline$P_{\text {gas }}$ & Pressão total no volume ocupado pelos gases [bar] & 1,013 & 1,013 \\
\hline$p_{\text {gas, } \mathrm{h} 2}$ & Pressão do vapor de água [bar] & 0,055667745 & 0,05566775 \\
\hline$R$ & $\begin{array}{l}\text { Constante universal - lei dos gases ideais [bar } \mathrm{M}^{-1} \\
\mathrm{~K}^{-1} \text { ] }\end{array}$ & 0,083145 & 0,083145 \\
\hline$k_{\mathrm{p}}$ & Coeficiente de resistência para os gases $\left[\mathrm{m}^{3} \mathrm{~d}^{-1} \mathrm{bar}^{-1}\right]$ & $5,00 \times 10^{4}$ & $5,00 \times 10^{4}$ \\
\hline
\end{tabular}


Tabela 16 - Parâmetros para simulação do modelo ADM1 completo (continua)

(a) Taxas de processos bioquímicos extracelulares de ordem 1: hidrólise $\left[\mathrm{d}^{-1}\right]$

\begin{tabular}{llc}
\hline$k_{\text {dis }}$ & Taxa de desintegração para quebra dos compostos complexos & 0,5 \\
$k_{\text {hyd,ch }}$ & Taxa de hidrólise de carboidratos para formação de monossacarídeos & 10 \\
$k_{\text {hyd,li }}$ & Taxa de hidrólise de lipídios para formação de ácidos graxos & 10 \\
$k_{\text {hyd,pr }}$ & Taxa de hidrólise de proteínas para formação de aminoácidos & 10 \\
\hline
\end{tabular}

(b) Constantes de cinética biológica relacionada à máxima taxa de crescimento bacteriano (para cada grupo de bactérias) $\left[\mathrm{d}^{-1}\right]$

\begin{tabular}{llc}
\hline$k_{\mathrm{m}, \mathrm{aa}}$ & Biomassa consumidora de aminoácido & 50 \\
$k_{\mathrm{m}, \mathrm{fa}}$ & Biomassa consumidora de ácidos graxos & 6 \\
\hline
\end{tabular}

(c) Taxas de produção de biomassa (para cada grupo de bactérias) [adimensional]

\begin{tabular}{lll}
\hline$Y_{\text {aa }}$ & Biomassa consumidora de aminoácido & 0,08 \\
$Y_{\mathrm{fa}}$ & Biomassa consumidora de ácidos graxos & 0,06 \\
\hline
\end{tabular}

(d) Constantes de Monod ou valor de meia saturação (para cada grupo de bactérias) [kg-DQO m ${ }^{-3}$ ]

\begin{tabular}{lll}
\hline$K_{\mathrm{S}, \text { aa }}$ & Biomassa consumidora de aminoácido & 0,3 \\
$K_{\mathrm{S}, \mathrm{fa}}$ & Biomassa consumidora de ácidos graxos & 0,3 \\
\hline
\end{tabular}

(e) Taxas de decaimento (para cada grupo de bactérias) $\left[\mathrm{d}^{-1}\right]$

Tabela 17 - Parâmetros para simulação do modelo ADM1 completo (conclusão)

\begin{tabular}{lll}
\hline$k_{\text {dec,Xaa }}$ & Biomassa consumidora de aminoácido & 0,02 \\
$k_{\text {dec,Xfa }}$ & Biomassa consumidora de ácidos graxos & 0,02 \\
\hline
\end{tabular}

(f) Concentrações de hidrogênio que inibem em $50 \%$ certos grupos de bactérias [kg-DQO m ${ }^{-3}$ ]

\begin{tabular}{lll}
\hline$K_{\mathrm{I}, \mathrm{h} 2 \text {,fa }}$ & Bactérias que consomem ácidos graxos & $5,00 \times 10^{-6}$ \\
\hline
\end{tabular}

( $g$ ) Quantidade de carbono nos componentes [ $\left.\mathrm{kmol} \mathrm{kg-DQO}^{-1}\right]$

\begin{tabular}{lll}
\hline$C_{\text {aa }}$ & Nos aminoácidos & 0,03 \\
\hline
\end{tabular}

(h) Quantidade de nitrogênio nos componentes [ $\left.\mathrm{kmol} \mathrm{kg-DQO}{ }^{-1}\right]$

\begin{tabular}{lll}
\hline$N_{\text {aa }} \quad$ Nos aminoácidos e proteínas & 0,007 \\
\hline
\end{tabular}


(i) Parâmetros estequiométricos das reações bioquímicas [adimensionais]

$f_{\mathrm{SI}, \mathrm{xc}} \quad$ Taxa de formação de solúveis inertes obtidos do consumo de compostos $\mathrm{Xc}$

0,1

$f_{\mathrm{XI}, \mathrm{xc}}$ Taxa de formação de particulados inertes obtidos do consumo de compostos Xc $\quad 0,2$

$f_{\text {ch,xc }}$ Taxa de formação de carboidratos obtidos do consumo de compostos Xc $\quad 0,2$

$f_{\mathrm{pr}, \mathrm{xc}} \quad$ Taxa de formação de proteínas obtidas do consumo de compostos Xc $\quad 0,2$

$f_{\text {li,xc }} \quad$ Taxa de formação de lipídios obtidos do consumo de compostos Xc $\quad 0,3$

$f_{\text {fa,li }} \quad$ Taxa de formação de ácidos graxos obtidos do consumo de lipídios $\quad 0,95$

$f_{\text {h2,aa }}$ Taxa de formação de gás hidrogênio obtido do consumo de aminoácidos $\quad$ 0,06

$f_{\text {va,aa }}$ Taxa de formação de valerato obtido do consumo de monossacarídeos $\quad 0,23$

$f_{\text {bu,aa }}$ Taxa de formação de butirato obtido do consumo de aminoácidos $\quad 0,26$

$f_{\text {pro,aa }}$ Taxa de formação de propionato obtido do consumo de aminoácidos $\quad 0,05$

$f_{\text {ac,aa }}$ Taxa de formação de acetato obtido do consumo de aminoácidos $\quad 0,4$

Fonte: Própria autoria. 


\section{RESULTADOS}

\subsection{Implementação inicial - teste}

Para fins de treinamento e verificação da funcionalidade do algoritmo, inicialmente foi implementado o modelo ADM1 em Python utilizando apenas 6 espécies, listadas na Tabela 17. Tal implementação, relativamente mais simples, baseou-se no desenvolvimento de um simulador numérico para biorreator via método de Boltzmann em rede (lattice Boltzmann method - LBM) em uma dimensão (FORTUNATO et al., 2018).

Tabela 18 - Nomenclatura e descrição das espécies consideradas

\begin{tabular}{ccc}
\hline $\boldsymbol{C}_{\boldsymbol{n}}$ (espécie química) & Descrição da espécie química & Unidades \\
\hline$C_{1}$ & demanda química de oxigênio (DQO) & $\mathrm{kg}^{-\mathrm{DQO} \mathrm{m}}{ }^{-3}$ \\
$C_{2}$ & ácido acético & $\mathrm{kg-DQO} \mathrm{m}$ \\
$C_{3}$ & ácido propiônico & $\mathrm{kg-DQO} \mathrm{m}$ \\
$C_{4}$ & ácido butírico & $\mathrm{kg}^{-3} \mathrm{DQO} \mathrm{m}$ \\
$C_{5}$ & hidrogênio dissolvido $\left(\mathrm{H}_{2}\right)$ & $\mathrm{kg}^{-3} \mathrm{DQO} \mathrm{m}$ \\
$C_{7}$ & biomassa & $\mathrm{kg}^{-3} \mathrm{DQO} \mathrm{m}$ \\
\hline
\end{tabular}

Fonte: FORTUNATO et al., 2018

Em Fortunato et al. (2018) foi considerado um reator anaeróbio em leito fixo (APBR anaerobic packed bed reactor) com fluxo ascendente contínuo de efluente. O modelo elaborado foi dinâmico e unidimensional (1-D), com o simulador LBM (em Fortran) capaz de fornecer as concentrações das espécies químicas (restritas à Tabela 18) não apenas em função do tempo mas também ao longo da coordenada vertical (eixo central) do APBR.

Assim, para fins de comparação com simulações iniciais no presente trabalho, foram consideradas as concentrações simuladas em FORTUNATO et al. (2018) junto à saída do biorreator ao longo de 1 dia, mantidas as mesmas condições operacionais do APBR, que se tratava de um reator específico para produção de hidrogênio, onde não ocorre remoção de DQO. Tais simulações iniciais (em Python) fornecem as concentrações das espécies ao longo do tempo na saída de um biorreator CSTR. Para cada espécie química da Tabela 18, as Figuras $4(a)$ - $(f)$ comparam as concentrações de saída ao longo de 1 dia de operação (tratamento).

As diferenças observadas entre as concentrações podem ser justificadas pelo fato de serem simulados diferentes tipos de biorreatores anaeróbios, a partir de modelos dinâmicos 
distintos, sem dependência espacial (CSTR) e dependência unidimensional (APBR). Ainda assim, as concentrações simuladas na saída de cada biorreator são fisicamente coerentes. 
Figura 4 - Perfis de concentração comparativos da simulação de Fortunato et al. (2018) em 1 D, na saída do reator versus resultados das simulações iniciais deste trabalho para cada espécie
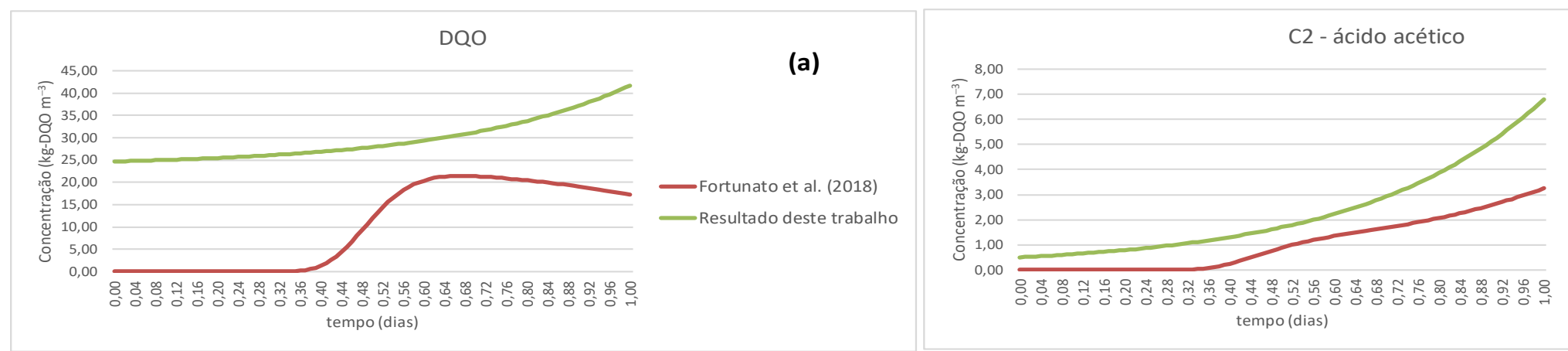

(b)
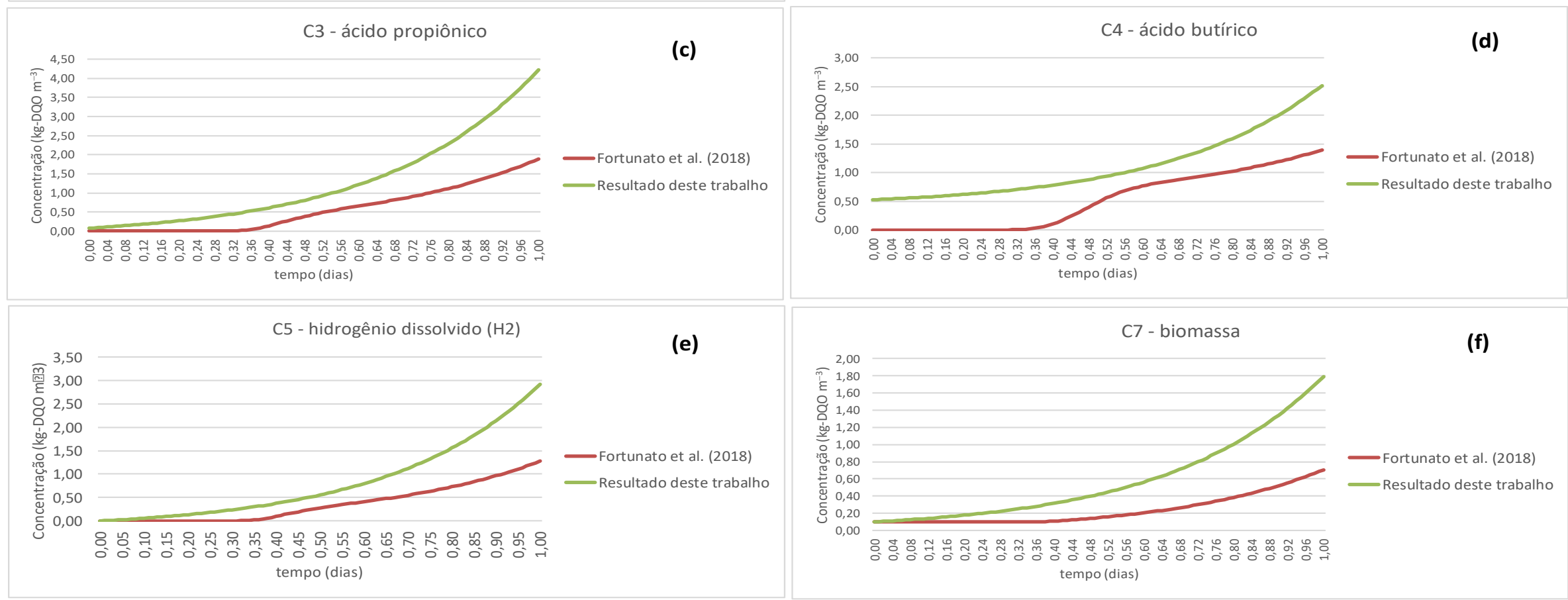

Fonte: Fortunato et al. (2018) e própria autoria. 
Além de treinamento inicial em modelagem fenomenológica e simulação numérica de biorreatores, este simulador preliminar (em Python) foi base para a elaboração dos demais simuladores descritos a seguir. Para tais simuladores numéricos subsequentes, vale ressaltar que foi feita a extensão do código computacional de 6 para 35 espécies químicas e para 40 espécies no ADM1 completo.

\subsection{Simulação 1-A: Verificação da implementação}

O objetivo principal foi confrontar os valores simulados pela implementação utilizando a linguagem Python de programação contra os obtidos nos trabalhos utilizados como base para este. Os valores adotados para as variáveis na alimentação, assim como as estimativas iniciais, seguiram os utilizados por Danielsson (2014), declarados na Tabela 18.

Vale recapitular que neste treinamento não foram consideradas todas as etapas do processo de digestão anaeróbia do modelo ADM1 mas apenas as rotas de maior relevância para o processo de tratamento da vinhaça de cana de açúcar. Desta forma, não era esperado que os resultados numéricos fossem fidedignos aos encontrados por Rosen e Jeppsson (2006) e Danielsson (2014). A simulação foi feita para um período de 50 dias, assim como foi feito por Danielsson (2014) e com 800.000 iterações.

Para cada espécie ora consideradas, as Figuras 5 a 8 mostram os respectivos perfis de concentração simulados ao longo do tempo. A nomenclatura utilizada para as variáveis nestas figuras remetem à declaração feita no código de programação (por isto, foram utilizados caracteres como “_”).

Para as espécies solúveis açúcares, valerato, butirato, propionato, acetato e hidrogênio, Figura 5(a)-(f), os perfis de concentração apresentaram certa similaridade com os resultados reportados por Danielsson (2014) enquanto que as concentrações de metano, carbono e nitrogênio inorgânicos apresentaram comportamento diferente. Mostradas na Figura 6(b)-(f), as biomassas consumidoras de açúcares, butirato e valerato, propionato, acetato e hidrogênio, também tiveram comportamento diferente dos perfis encontrados por Danielsson (2014). No entanto, o simulador foi capaz de reproduzir todos estes perfis sem instabilidades numéricas.

$\mathrm{Na}$ simulação das concentrações dos ácidos valérico, butírico, propiônico e acético ocorreram oscilações nos valores numéricos representadas pela área preenchida abaixo do perfil, Figura 7(a)-(d). Analogamente, foram observadas oscilações na simulação (após 20 dias de processo) para as espécies bicarbonato, amônia, gás hidrogênio gás metano e dióxido de carbono, apresentadas na Figura 7(e)-(h) e Figura 8(a) respectivamente. 
Tabela 19 - Valores adotados para as concentrações das diferentes espécies na alimentação e estimativas iniciais para esta simulação: 1 - A

\begin{tabular}{|c|c|c|c|}
\hline Espécie & Unidade & Alimentação & Estimativa inicial \\
\hline$s_{\text {su }}$ & kg-DQO.m ${ }^{-3}$ & 0,01 & 0,009 \\
\hline$s_{\mathrm{va}}$ & $\mathrm{kg}-\mathrm{DQO} \cdot \mathrm{m}^{-3}$ & 0,001 & 0,0009 \\
\hline${ }^{S_{\mathrm{bu}}}$ & $\mathrm{kg}-\mathrm{DQO} \cdot \mathrm{m}^{-3}$ & 0,001 & 0,0009 \\
\hline$S_{\text {pro }}$ & kg-DQO. $\mathrm{m}^{-3}$ & 0,001 & 0,0009 \\
\hline$s_{\mathrm{ac}}$ & $\mathrm{kg}-\mathrm{DQO} \cdot \mathrm{m}^{-3}$ & 0,001 & 0,00009 \\
\hline${ }^{s} \mathrm{~h} 2$ & kg-DQO. $\mathrm{m}^{-3}$ & $1,00 \mathrm{E}-08$ & 0,000023594 \\
\hline$S_{\operatorname{ch} 4}$ & $\mathrm{~kg}-\mathrm{DQO} \cdot \mathrm{m}^{-3}$ & $1,00 \mathrm{E}-05$ & 0,023594 \\
\hline${ }^{S} \mathrm{IC}$ & kmol-C.m ${ }^{-3}$ & 0,04 & 0,0390 \\
\hline${ }^{S} \mathrm{IN}$ & kmol-N.m ${ }^{-3}$ & 0,01 & 0,1302 \\
\hline$x_{\mathrm{su}}$ & $\mathrm{kg}-\mathrm{DQO} \cdot \mathrm{m}^{-3}$ & 0 & 0,4202 \\
\hline$x_{\mathrm{c} 4}$ & kg-DQO. $\mathrm{m}^{-3}$ & 0,01 & 0,4319 \\
\hline$x_{\mathrm{pr}}$ & $\mathrm{kg}-\mathrm{DQO} \cdot \mathrm{m}^{-3}$ & 0,01 & 0,1373 \\
\hline$x_{\mathrm{ac}}$ & kg-DQO. $\mathrm{m}^{-3}$ & 0,01 & 0,7606 \\
\hline${ }^{x_{\mathrm{h} 2}}$ & $\mathrm{~kg}-\mathrm{DQO} \cdot \mathrm{m}^{-3}$ & 0,01 & 0,3170 \\
\hline$s_{\text {cat }}$ & kg-DQO. $\mathrm{m}^{-3}$ & 0,04 & 0,0400 \\
\hline$s_{\text {an }}$ & $\mathrm{kg}-\mathrm{DQO} \cdot \mathrm{m}^{-3}$ & 0,02 & 0,0200 \\
\hline$S_{\text {vam }}$ & kg-DQO. $\mathrm{m}^{-3}$ & $X$ & 0,0116 \\
\hline${ }^{s}$ bum & kg-DQO. $\mathrm{m}^{-3}$ & $\mathrm{X}$ & 0,0132 \\
\hline$S_{\text {prom }}$ & kg-DQO. $\mathrm{m}^{-3}$ & $\mathrm{X}$ & 0,0157 \\
\hline$s_{\mathrm{acm}}$ & $\mathrm{kg}-\mathrm{DQO} \cdot \mathrm{m}^{-3}$ & $\mathrm{X}$ & 0,1972 \\
\hline${ }^{S}$ hco3m & kmol-C.m ${ }^{-3}$ & $\mathrm{X}$ & 0,1428 \\
\hline$s_{\mathrm{nh} 3}$ & kmol-N.m ${ }^{-3}$ & $X$ & 0,0041 \\
\hline$s_{\text {gas, } \mathrm{h} 2}$ & kg-DQO. $\mathrm{m}^{-3}$ & $\mathrm{X}$ & 0,1023 \\
\hline$s_{\text {gas,ch} 4}$ & kg-DQO. $\mathrm{m}^{-3}$ & $X$ & 16213,0 \\
\hline$s_{\text {gas,co } 2}$ & kmol-C.m ${ }^{-3}$ & $X$ & 0,0141 \\
\hline${ }^{S} \mathbf{I}$ & kg-DQO. $\mathrm{m}^{-3}$ & 0,02 & 0,0090 \\
\hline${ }^{X} \mathbf{I}$ & kg-DQO. $\mathrm{m}^{-3}$ & 25,0 & 256174,0 \\
\hline
\end{tabular}

$\mathrm{X}$ : concentração destas espécies na alimentação é considerada nula, entretanto não é necessário declaração dos valores na alimentação. Fonte: Própria autoria. 
Figura 5 - Perfil de concentração das espécies solúveis: açúcares (a), valerato (b), butirato (c), propionato (d) acetato (e) e hidrogênio (f), metano (g) e carbono inorgânico (h)
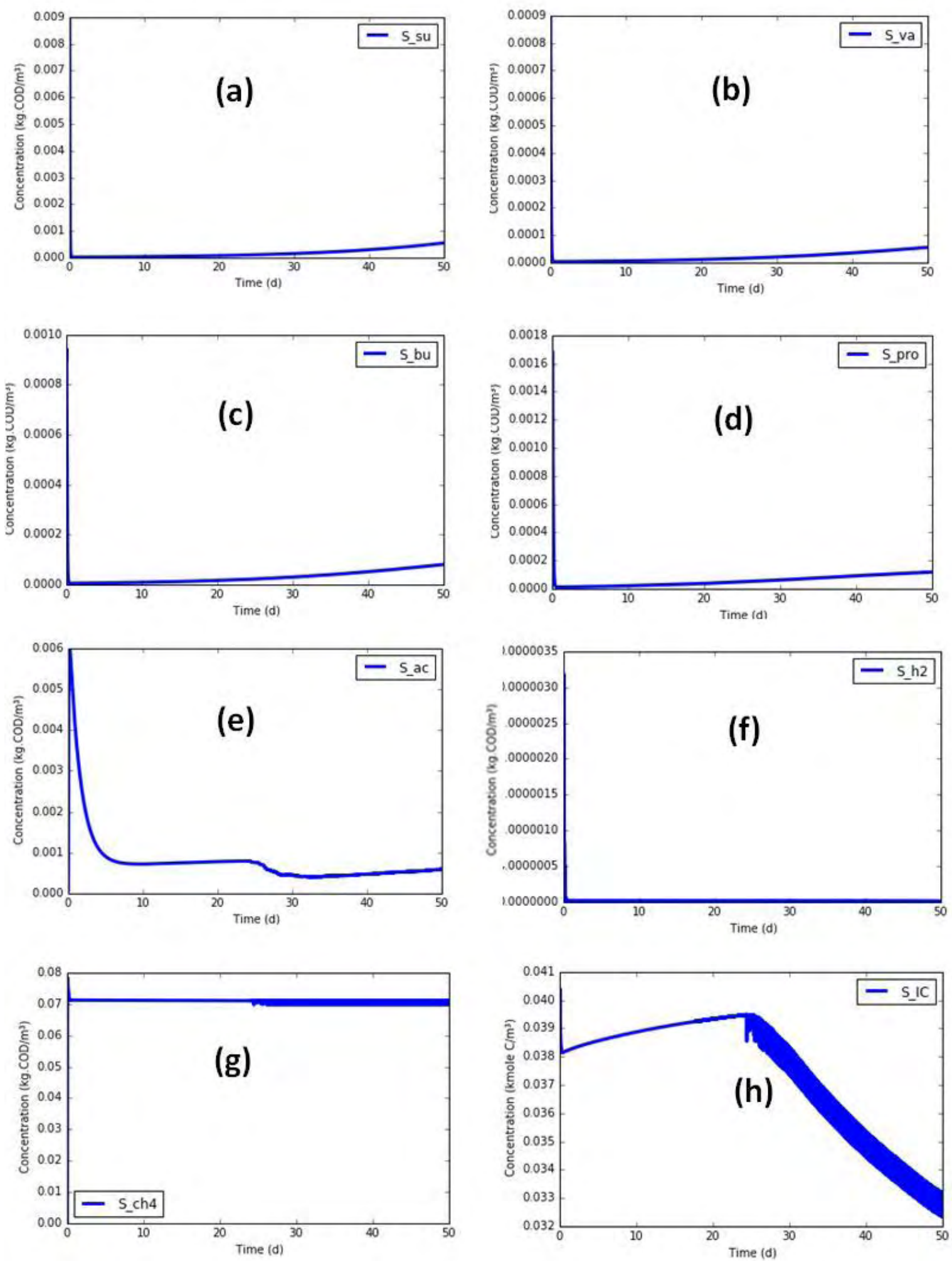

Fonte: Própria autoria. 
Figura 6 - Perfil de concentração das espécies: nitrogênio inorgânico solúvel (a), biomassa de açúcares (b), biomassa de butirato e valerato (c) e biomassa de propionato (d), biomassa de acetato (e), biomassa de hidrogênio (f), cátions (g), ânions (h)
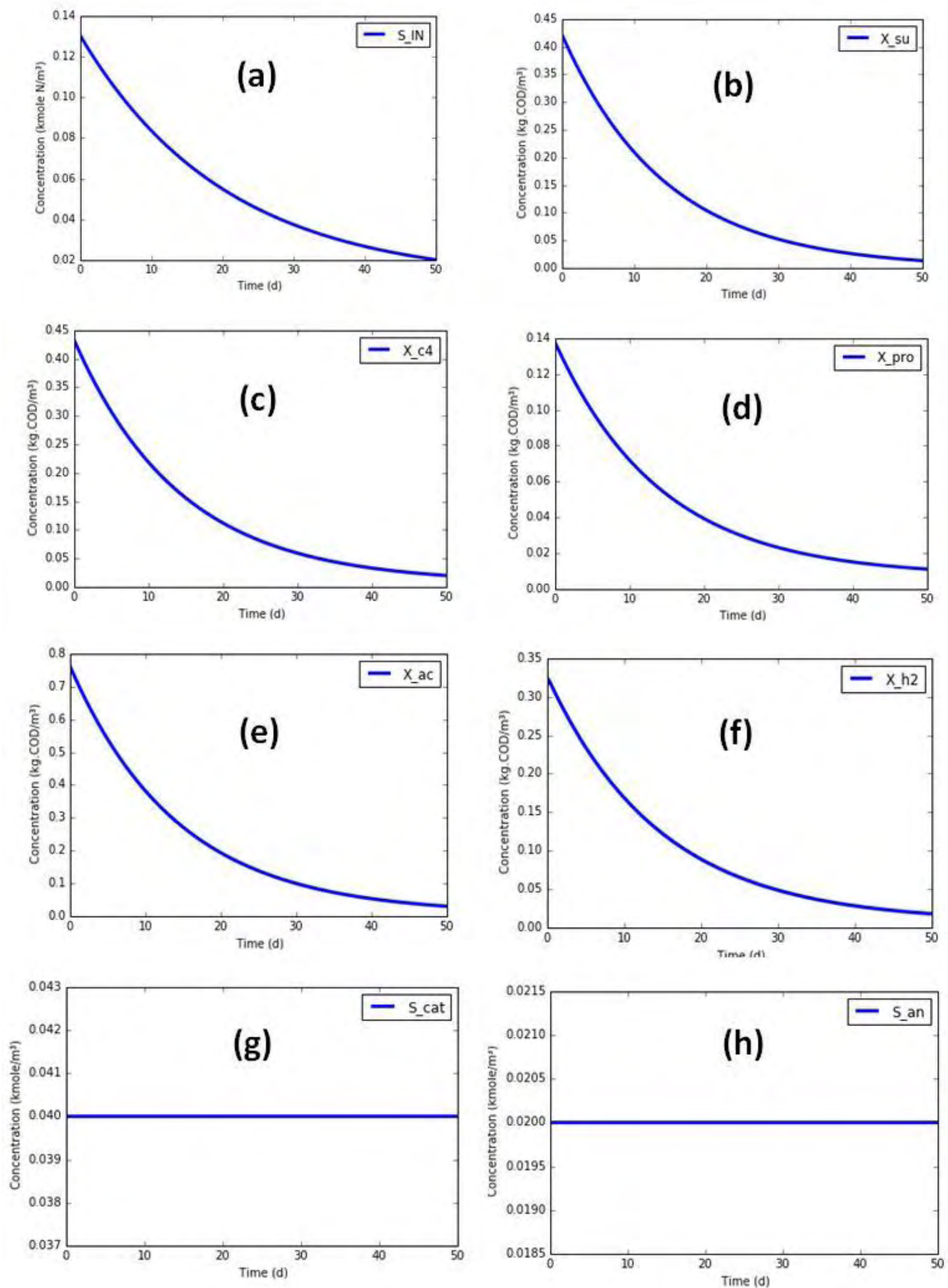

Fonte: Própria autoria. 
Figura 7 - Perfil de concentração das espécies solúveis: ácido valérico (a), ácido butírico (b), ácido propiônico (c), ácido acético (d), bicarbonato (e), amônia (f), gás hidrogênio (g) e gás metano (h)
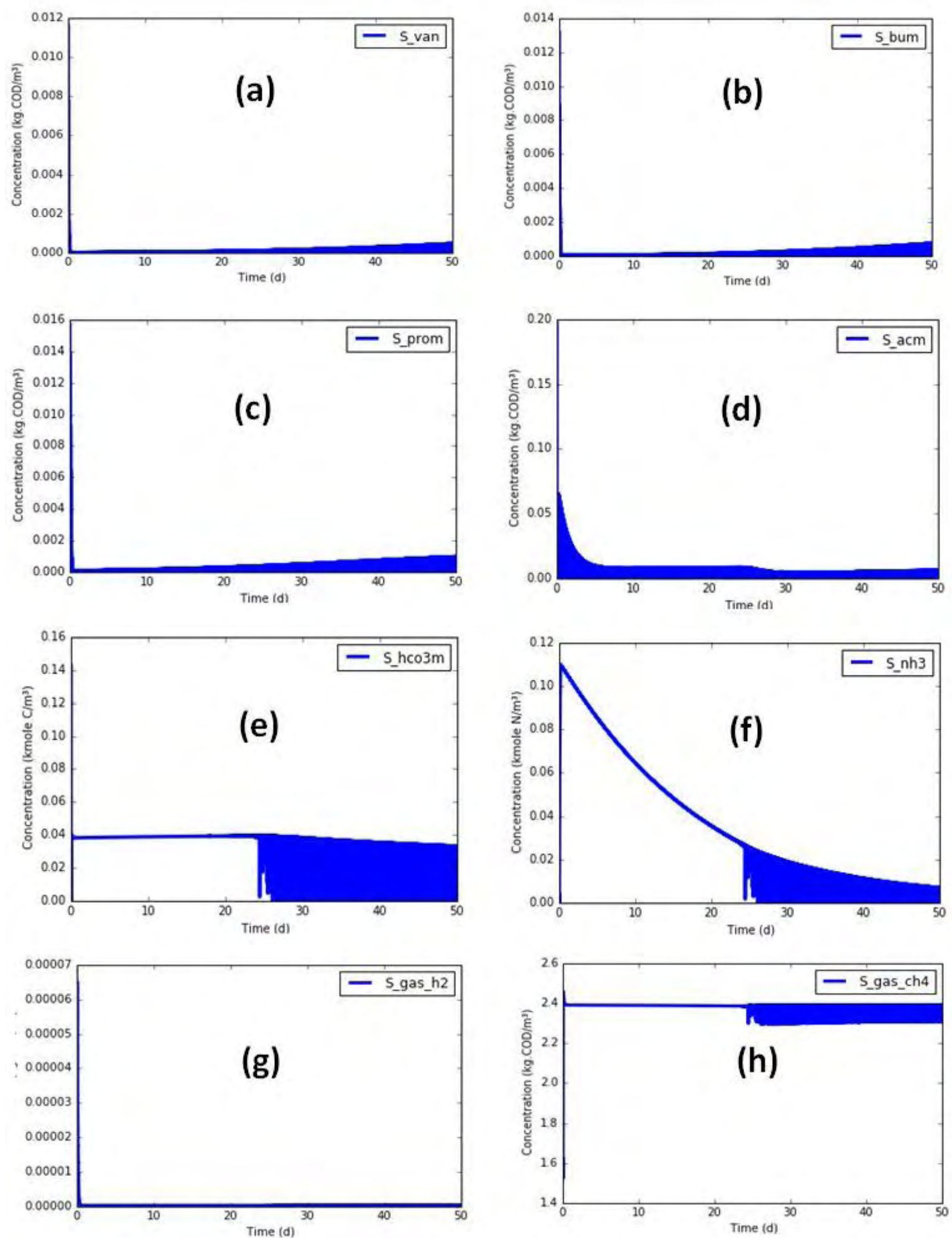

Fonte: Própria autoria. 
Figura 8 - Perfil de concentração das espécies: gás $\mathrm{CO}_{2}$ (a), cálcio (b), inerte solúvel (c), inerte particulado (d), lactato solúvel (e), lactato do processo de fermentação (f), lactato do processo de oxidação (g), DQO total (h)
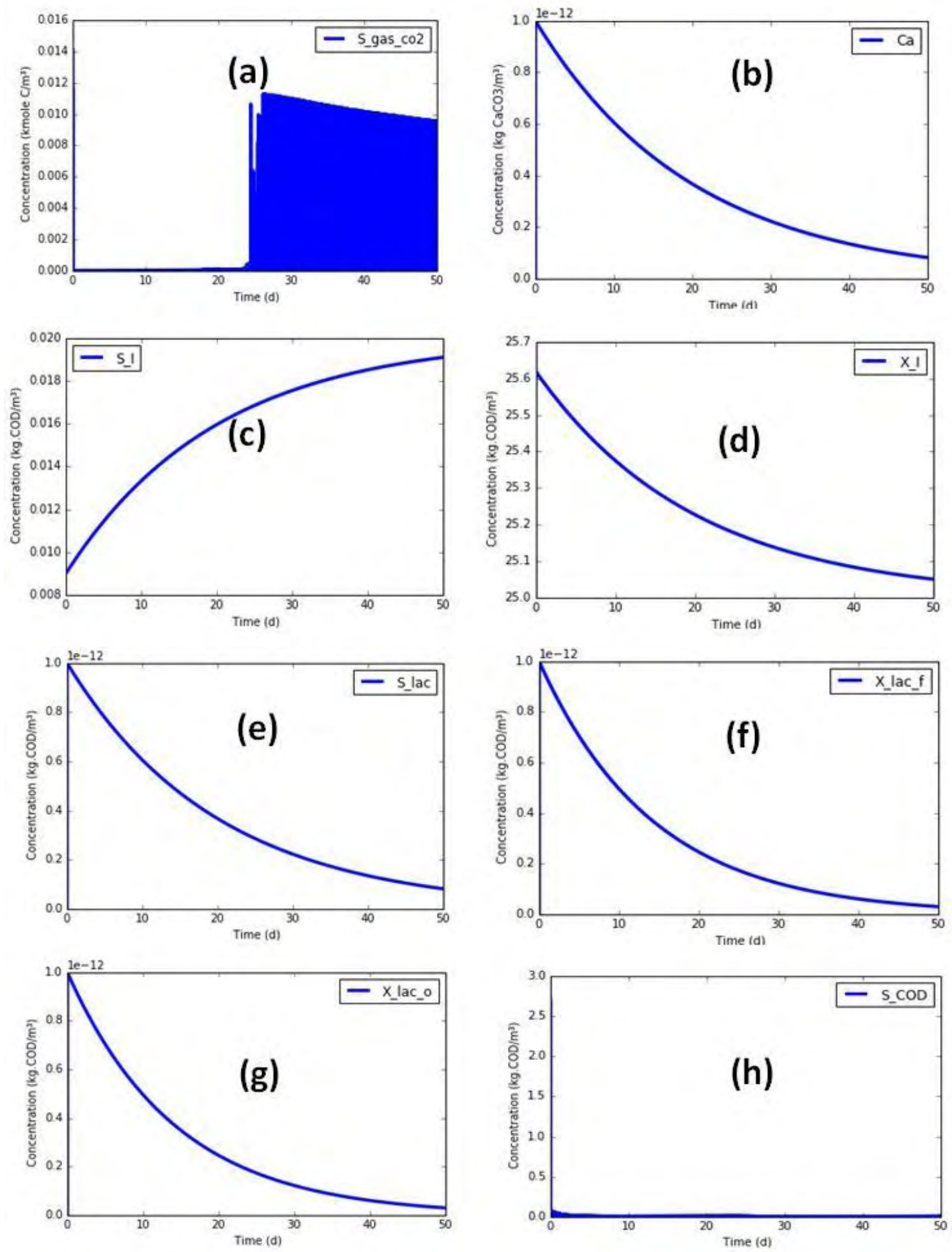

Fonte: Própria autoria. 
Antes de realizar a simulação com 800.000 iterações, foram testadas simulações mais curtas. Por exemplo, ocorreu divergência numérica na simulação com 8.000 iterações das concentrações de $\mathrm{H}_{2}, \mathrm{CH}_{4}$ e $\mathrm{CO}_{2}$, bicarbonato e carbono inorgânico. $\mathrm{O}$ código desenvolvido (com base na ferramenta "odeint" para resolução das EDOs) não teve sensibilidade numérica suficiente para simular tais concentrações. Mediante sua adequação para 800.000 iterações, o simulador se mostrou mais estável, ainda que com as divergências anteriormente discutidas.

Nas simulações, as concentrações de cálcio (Figura 8(b)) e lactatos (Figura 8(e)-(g)) apresentaram variações pequenas. Nas simulações feitas por Danielsson (2014) de 0 a 50 dias, as concentrações destas espécies não variaram no tempo. Por sua vez, as simulações da DQO e do pH apresentaram oscilações numéricas, Figura 8(h) e Figura 9 respectivamente.

Figura 9 - pH calculado pela Simulação 1

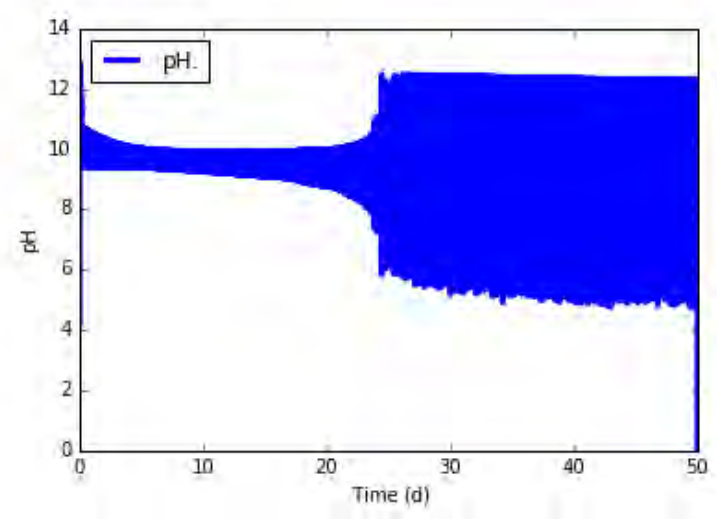

Fonte: Própria autoria.

Estas simulações preliminares (Simulação 1-A) evidenciaram dois desafios quanto à modelagem computacional de concentrações (de algumas espécies): reduzir as oscilações (instabilidades) numéricas e suavizar a redução rápida (em algumas horas) que comprometem simulações de processos mais longos (50 dias). Visando aumentar a estabilidade numérica pela redução do passo de tempo, novas simulações (Simulação 1-B) foram realizadas com 800.000 iterações, considerando um período menor de tratamento (5 dias). Foram adotados os valores na Tabela 18 para alimentação e estimativas iniciais da concentração de cada espécie.

\subsection{Simulação 1-B: Dados de entrada da Simulação 1-A mas 5 dias de tratamento}

As Figuras 10, 11 e 12 a seguir apresentam as concentrações simuladas (Simulação 1B) para as espécies químicas cujas concentrações não apresentaram oscilações na Simulação 
1-A. Nesta simulação foi possível, através da redução do passo de tempo, avaliar melhor o perfil das concentrações das espécies que tiveram uma redução rápida na acidogênese. Estes perfis foram simulados com maior estabilidade numérica, quando comparados aos resultados da simulação 1-A. 
Figura 10 - Perfil de concentração das espécies solúveis: açúcares (a), valerato (b), butirato (c), propionato (d) acetato (e) e hidrogênio (f), metano (g) e carbono inorgânico (h)
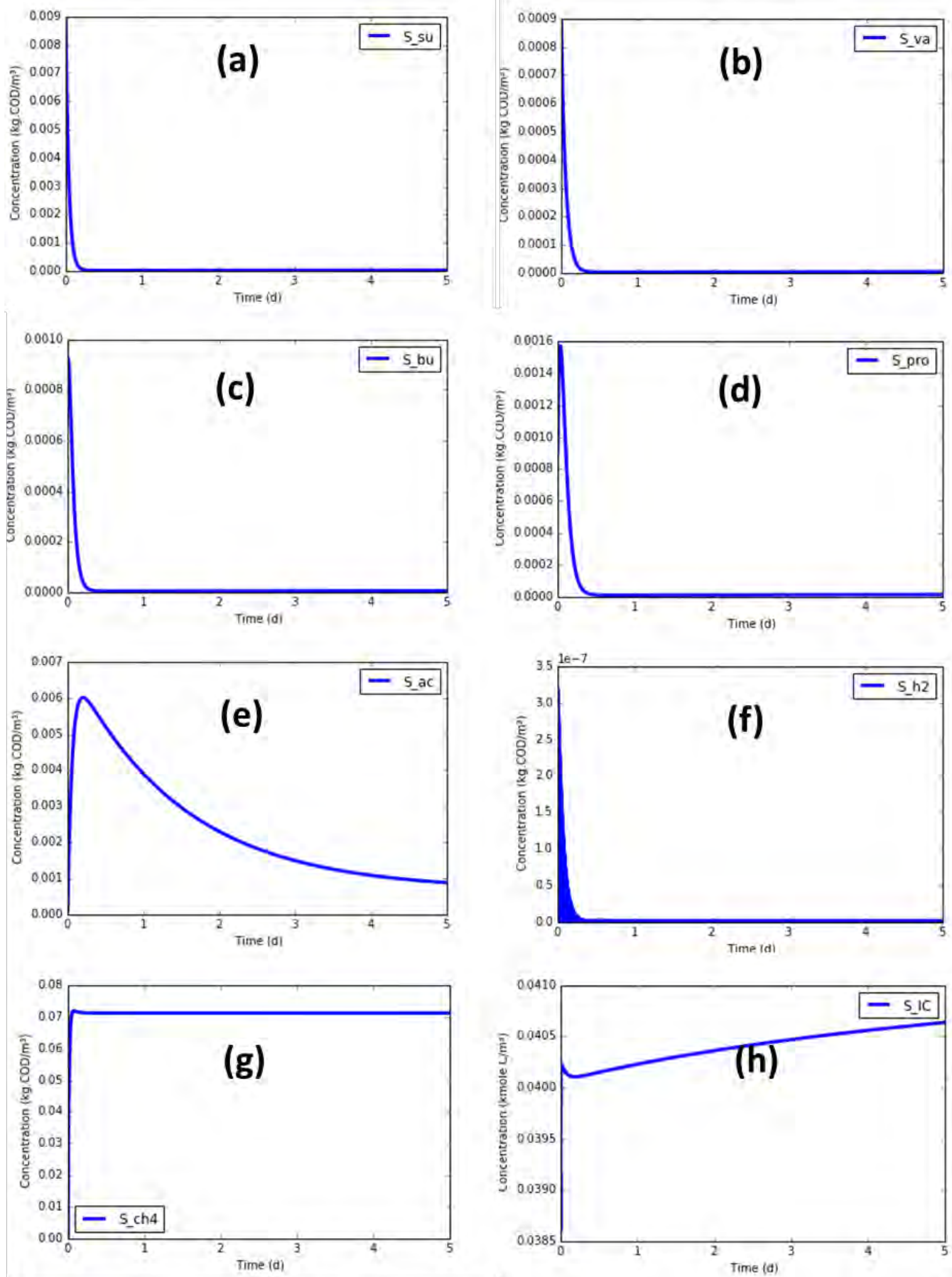

Fonte: Própria autoria. 
Figura 11 - Perfil de concentração das espécies: bicarbonato (a), amônia (b), gás hidrogênio (c), gás metano (d), gás carbônico (e) e DQO total (f)
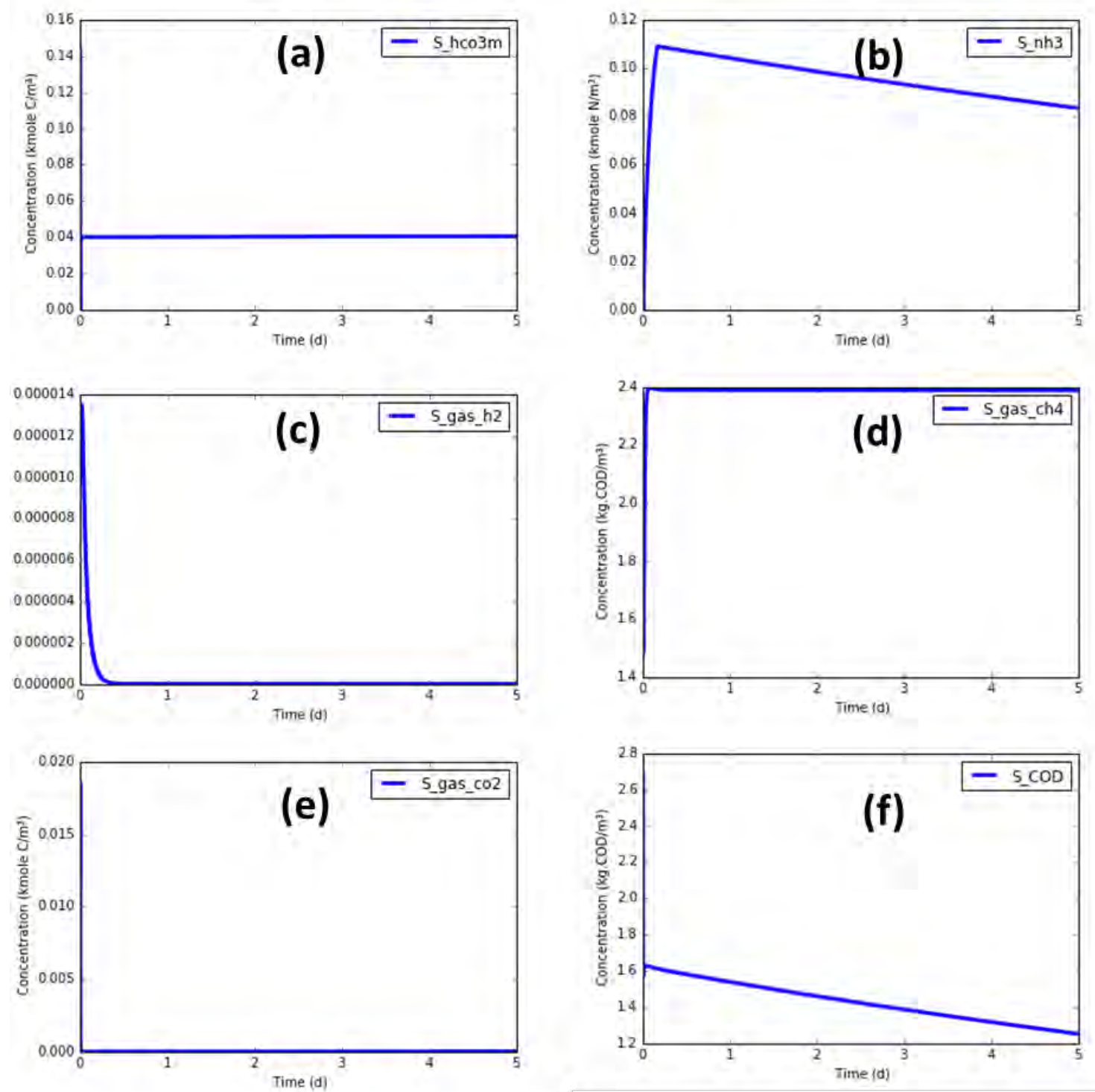

Fonte: própria autoria.

Figura 12 - pH calculado pela Simulação 1

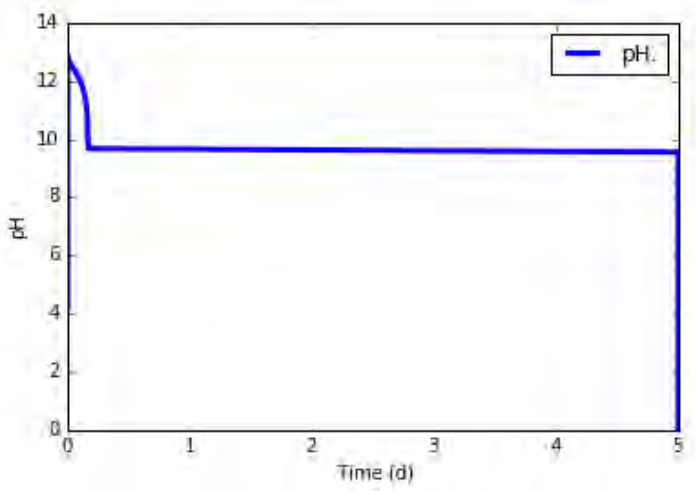

Fonte: Própria autoria. 


\subsection{Simulação 1-C: Alteração das estimativas inicias}

Esta simulação foi realizada com os parâmetros das Tabelas 3 a 15 (Simulação 1-A e 1-B), porém alterando os valores das estimativas inicias das concentrações de cada espécie, conforme a Tabela 20, para avaliação da convergência do simulador, partindo de outro ponto inicial. Considerou-se o mesmo tempo de 50 dias e 800.000 iterações. Os resultados obtidos nesta simulação estão descritos nas Figuras 13 a 17 a seguir.

Tabela 20 - Estimativas iniciais das concentrações das diferentes espécies da Simulação 1-C

\begin{tabular}{|c|c|c|}
\hline Espécie & Unidade & Estimativa inicial \\
\hline$s_{\text {su }}$ & kg-DQO.m ${ }^{-3}$ & 1,15 \\
\hline$s_{\mathrm{va}}$ & kg-DQO. $\mathrm{m}^{-3}$ & 0,052 \\
\hline$s_{\mathrm{bu}}$ & $\mathrm{kg}-\mathrm{DQO} \cdot \mathrm{m}^{-3}$ & 0,053 \\
\hline$S_{\text {pro }}$ & kg-DQO. $\mathrm{m}^{-3}$ & 0,123 \\
\hline$s_{\mathrm{ac}}$ & $\mathrm{kg}-\mathrm{DQO} \cdot \mathrm{m}^{-3}$ & 0,171 \\
\hline$s_{\mathrm{h} 2}$ & kg-DQO. $\mathrm{m}^{-3}$ & $1,0 \mathrm{E}-09$ \\
\hline$s_{\operatorname{ch} 4}$ & $\mathrm{~kg}-\mathrm{DQO} \cdot \mathrm{m}^{-3}$ & $1,0 \mathrm{E}-06$ \\
\hline${ }^{S} \mathrm{IC}$ & kmol-C.m ${ }^{-3}$ & 0,152677871 \\
\hline${ }^{s} \mathrm{IN}$ & kmol-N.m ${ }^{-3}$ & 0,13023 \\
\hline$x_{\text {su }}$ & $\mathrm{kg}-\mathrm{DQO} \cdot \mathrm{m}^{-3}$ & 0,012016 \\
\hline$x_{\mathrm{c} 4}$ & kg-DQO.m ${ }^{-3}$ & 0,01 \\
\hline${ }^{x_{p r}}$ & kg-DQO. $\mathrm{m}^{-3}$ & 0,003 \\
\hline$x_{\mathrm{ac}}$ & kg-DQO. $\mathrm{m}^{-3}$ & 0,04126 \\
\hline$x_{\mathrm{h} 2}$ & kg-DQO.m ${ }^{-3}$ & 0,31702 \\
\hline$s_{\text {cat }}$ & kg-DQO. $\mathrm{m}^{-3}$ & 0,04 \\
\hline$s_{\text {an }}$ & kg-DQO.m ${ }^{-3}$ & 0,02 \\
\hline$S_{\text {vam }}$ & $\mathrm{kg}-\mathrm{DQO} \cdot \mathrm{m}^{-3}$ & 0,052 \\
\hline$S_{\text {bum }}$ & kg-DQO. $\mathrm{m}^{-3}$ & 0,053 \\
\hline$S_{\text {prom }}$ & kg-DQO. $\mathrm{m}^{-3}$ & 0,123 \\
\hline$s_{\mathrm{acm}}$ & $\mathrm{kg}-\mathrm{DQO} \cdot \mathrm{m}^{-3}$ & 0,171 \\
\hline${ }^{s}$ hco $3 \mathrm{~m}$ & kmol-C.m $\mathrm{m}^{-3}$ & 0,142777479 \\
\hline${ }^{S}$ nh3 & kmol-N.m ${ }^{-3}$ & 0,00409 \\
\hline${ }^{s_{\text {gas }, \mathrm{h} 2}}$ & kg-DQO.m ${ }^{-3}$ & 0,00001 \\
\hline${ }^{s}$ gas,ch4 & kg-DQO. $\mathrm{m}^{-3}$ & 0,00001 \\
\hline$s_{\text {gas,co } 2}$ & kmol-C. $\mathrm{m}^{-3}$ & 0,00001 \\
\hline${ }^{s} \mathbf{I}$ & kg-DQO.m ${ }^{-3}$ & 0,009 \\
\hline${ }^{x_{I}}$ & kg-DQO. $\mathrm{m}^{-3}$ & 25,61739 \\
\hline
\end{tabular}

Fonte: Própria autoria. 
Figura 13 - Perfil de concentração das espécies solúveis: açúcares (a), valerato (b), butirato (c), propionato (d) acetato (e) e hidrogênio (f), metano (g) e carbono inorgânico (h)
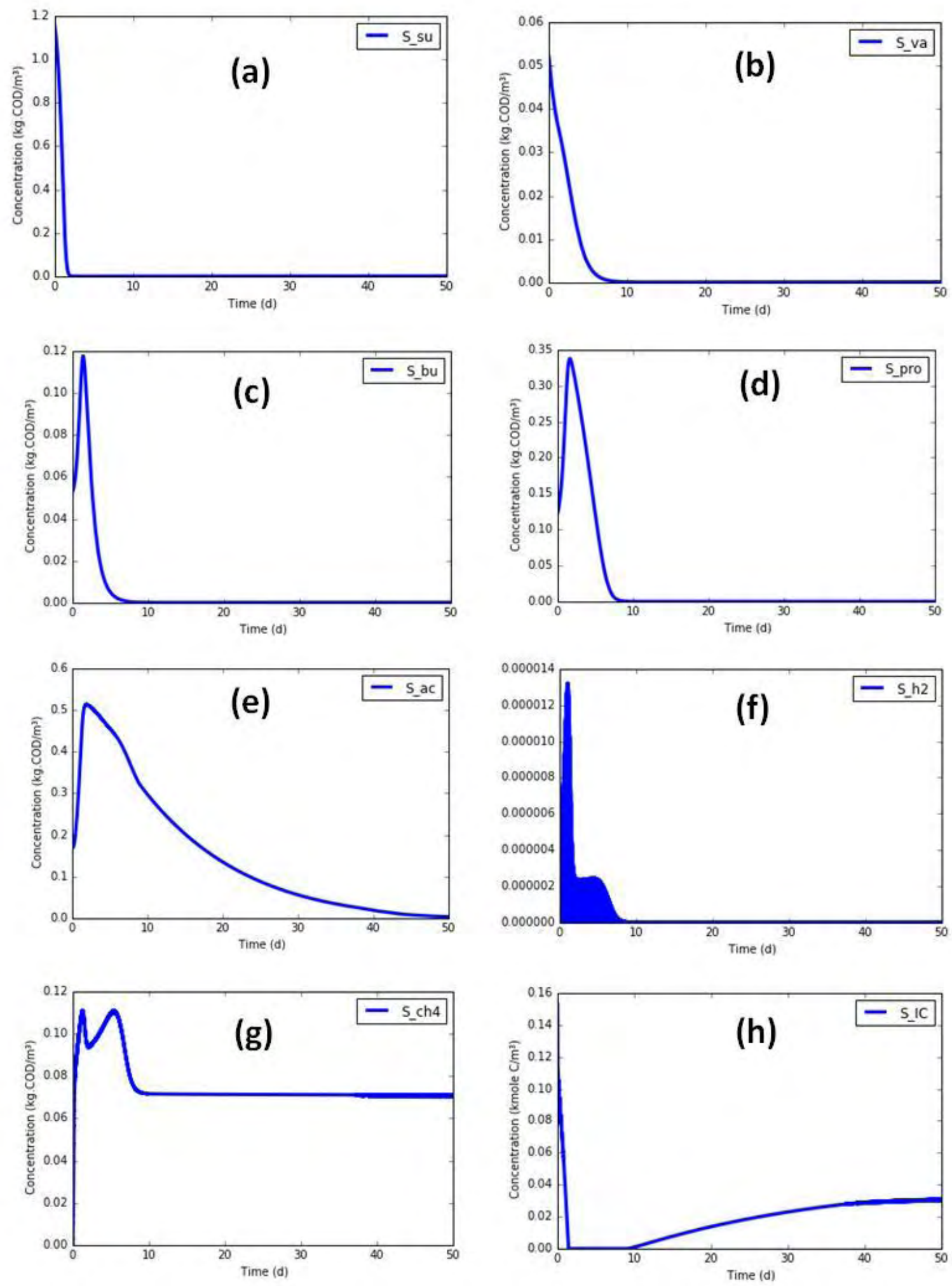

Fonte: Própria autoria. 
Figura 14 - Perfil de concentração das espécies: nitrogênio inorgânico solúvel (a), biomassa de açúcares (b), biomassa de butirato e valerato (c) e biomassa de propionato (d), biomassa de acetato (e), biomassa de hidrogênio (f), cátions (g), ânions (h)
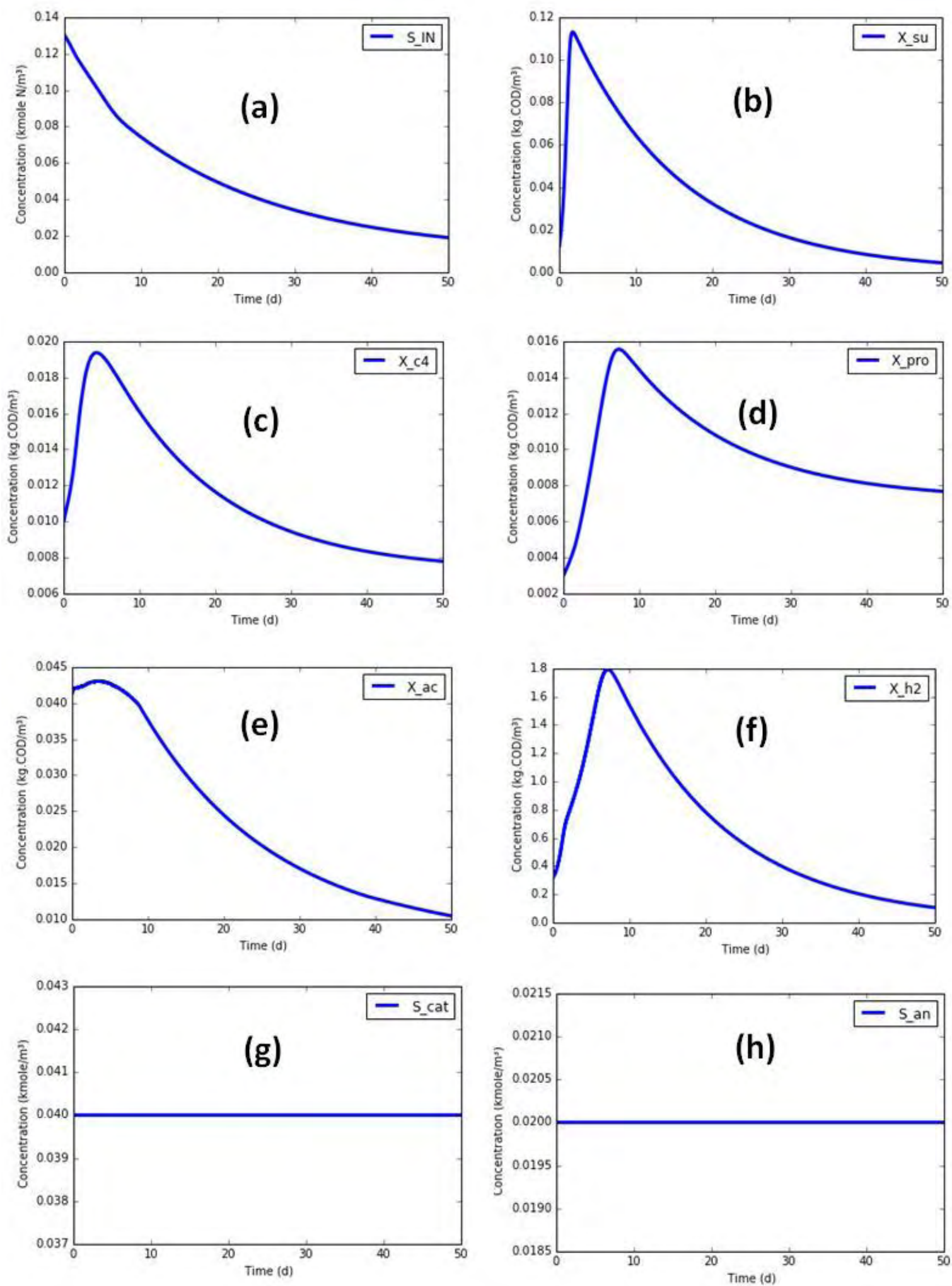

Fonte: Própria autoria. 
Figura 15 - Perfil de concentração das espécies solúveis: ácido valérico(a), ácido butírico (b), ácido propiônico (c), ácido acético (d), bicarbonato (e), amônia (f), gás $\mathrm{H}_{2}$ (g) e gás $\mathrm{CH}_{4}(\mathrm{~h})$
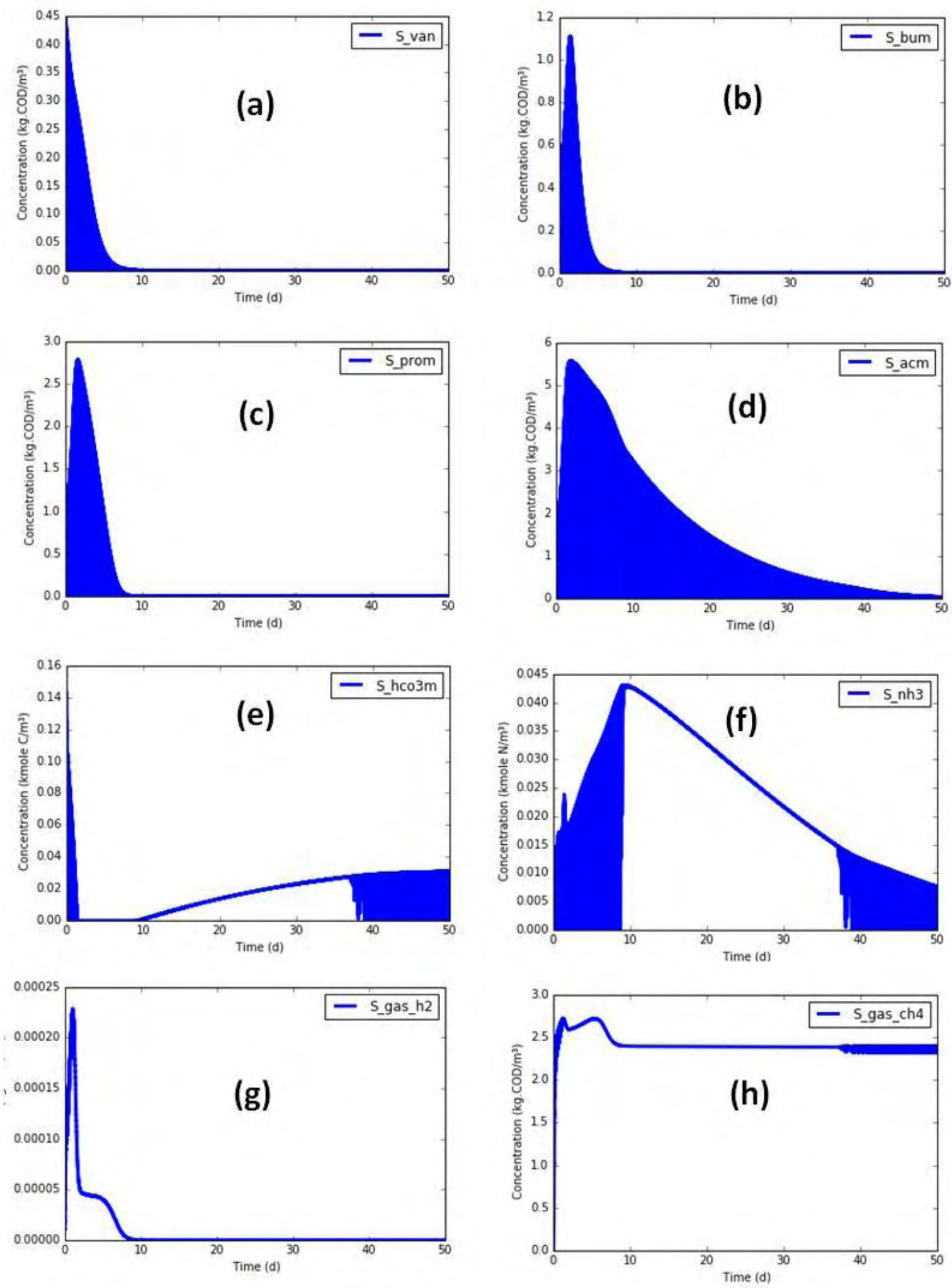

Fonte: Própria autoria. 
Figura 16 - Perfil de concentração das espécies: gás $\mathrm{CO}_{2}(\mathrm{a})$, cálcio (b), inerte solúvel (c), inerte particulado (d), lactato solúvel (e), lactato do processo de fermentação (f), lactato do processo de oxidação (g), DQO total (h)
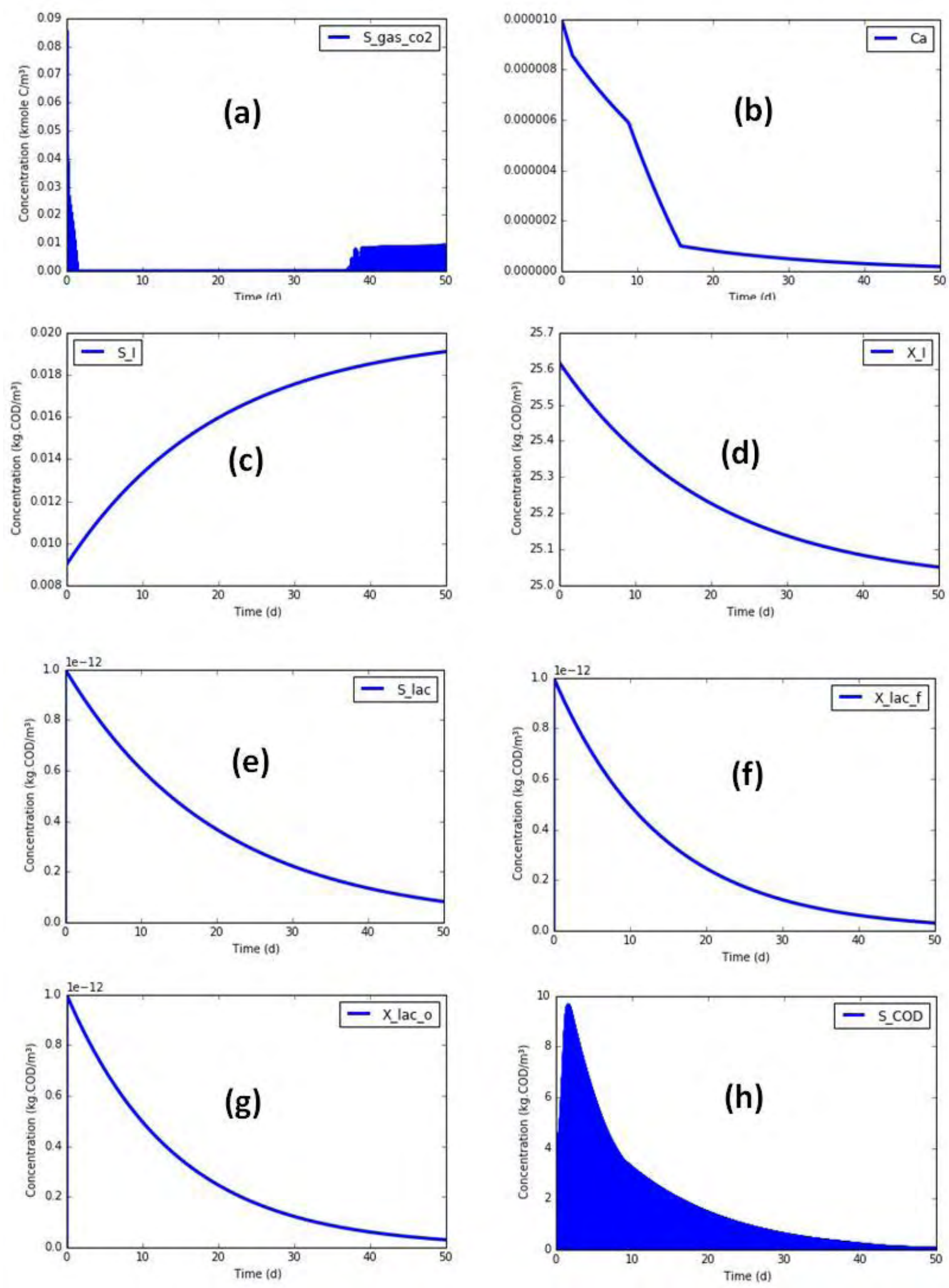

Fonte: Própria autoria. 
Figura 17 - pH calculado pela Simulação 1

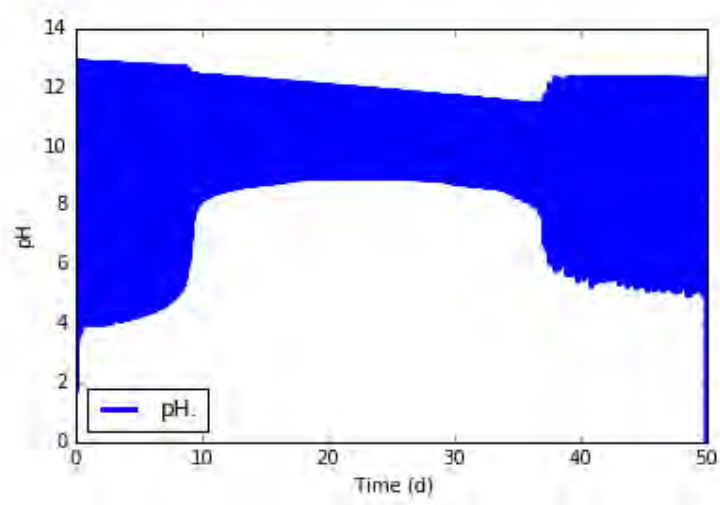

Fonte: Própria autoria.

A exemplo das simulações anteriores, os perfis de concentração das espécies solúveis foram adequadamente simulados, Figura 13(a)-(g), enquanto que para as espécies $\mathrm{H}_{2}$ e $\mathrm{CH}_{4}$ solúveis ainda houve certa instabilidade. Os perfis de concentração mostrados na Figura 14 foram simulados com estabilidade numérica mas os da Figura 15, notadamente nas Figuras 15(a)-(f), apresentaram oscilações numéricas (representadas pela área abaixo da curva).

As concentrações de $\mathrm{CO}_{2}$ e DQO, Figuras 16(a) e 16(h), apresentaram alguns períodos de oscilação numérica durante a simulação (área abaixo da curva), embora os respectivos perfis indiquem valores fisicamente aceitáveis. A concentração de cálcio e de DQO, Figuras 16(b) e 16(h) respectivamente, apresentaram comportamento muito diferente em comparação com os perfis na Simulação 1-A e 1-B. As concentrações de inertes solúvel e particulado, Figuras 16(c) e 16(d) respectivamente, bem como de lactatos, Figuras 16(e)-(g), apresentaram perfis semelhantes aos obtidos via Simulação 1-A e 1-B.

Embora fosse esperado que os perfis simulados fossem próximos (tendo em mente os parâmetros das Tabelas 18 e 19), foram observadas diferenças significativas para várias espécies. Tais diferenças foram atribuídas às instabilidades numéricas ocorridas ao longo do processo iterativo, de forma que foi conduzida uma análise mais detalhada tanto do modelo de simulação como do método numérico de solução, descrita a seguir.

\subsection{Simulação 2-A: Alteração dos parâmetros}

Nesta simulação foram considerados os parâmetros das Tabelas 3 a 15, os mesmos valores para as estimativas inicias das concentrações de cada espécie adotados na Simulação 1-C e o total de 800.000 iterações, por 50 dias. Os resultados numéricos obtidos estão apresentados nas Figuras 18 a 22. 
Figura 18 - Perfil de concentração das espécies solúveis: açúcares (a), valerato (b), butirato (c), propionato (d) acetato (e) e hidrogênio (f), metano (g) e carbono inorgânico (h)
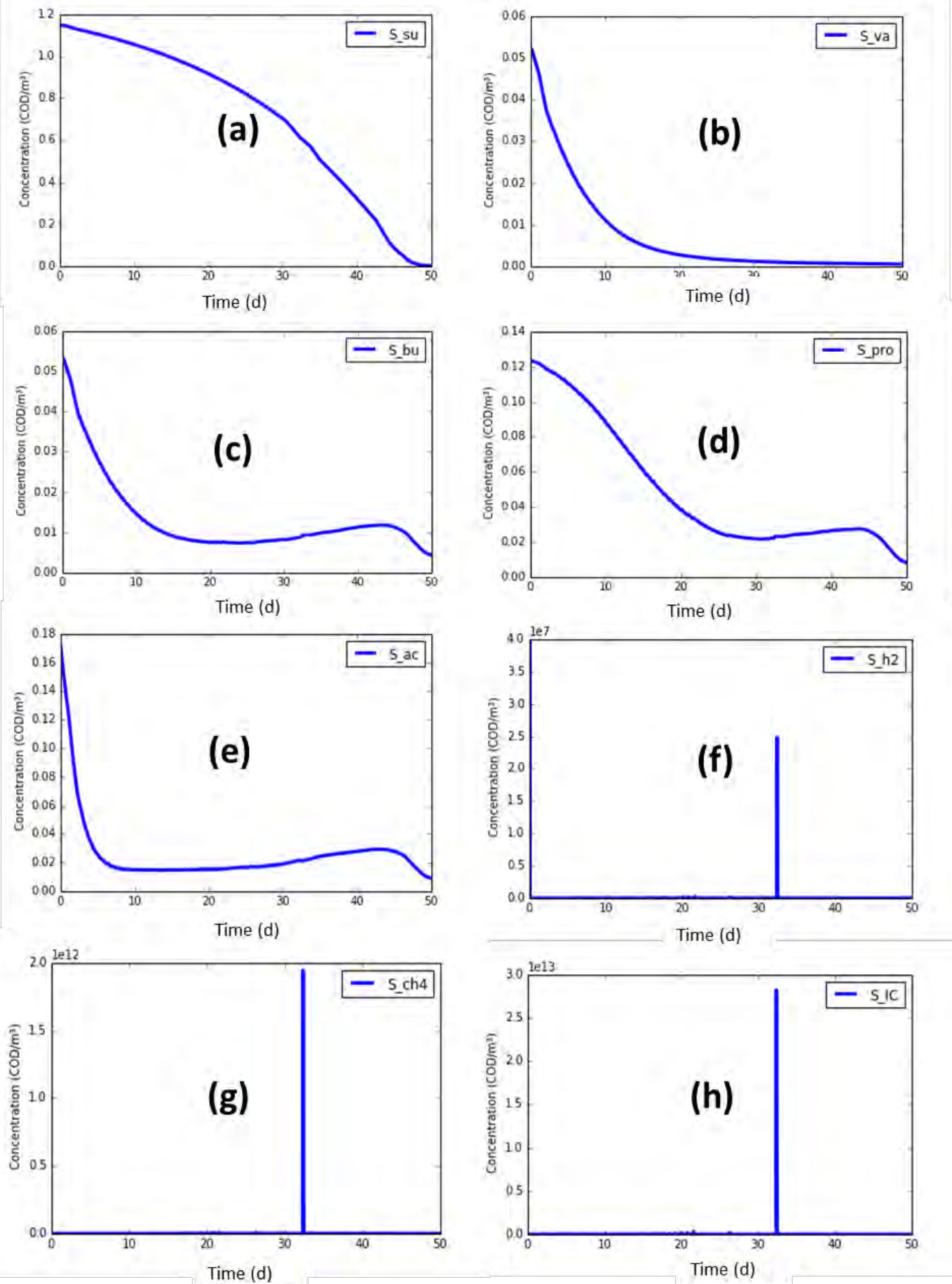

Fonte: Própria autoria. 
Figura 19 - Perfil de concentração das espécies: nitrogênio inorgânico solúvel (a), biomassa de açúcares (b), biomassa de butirato e valerato (c) e biomassa de propionato (d), biomassa de acetato (e), biomassa de hidrogênio (f), cátions (g), ânions (h)
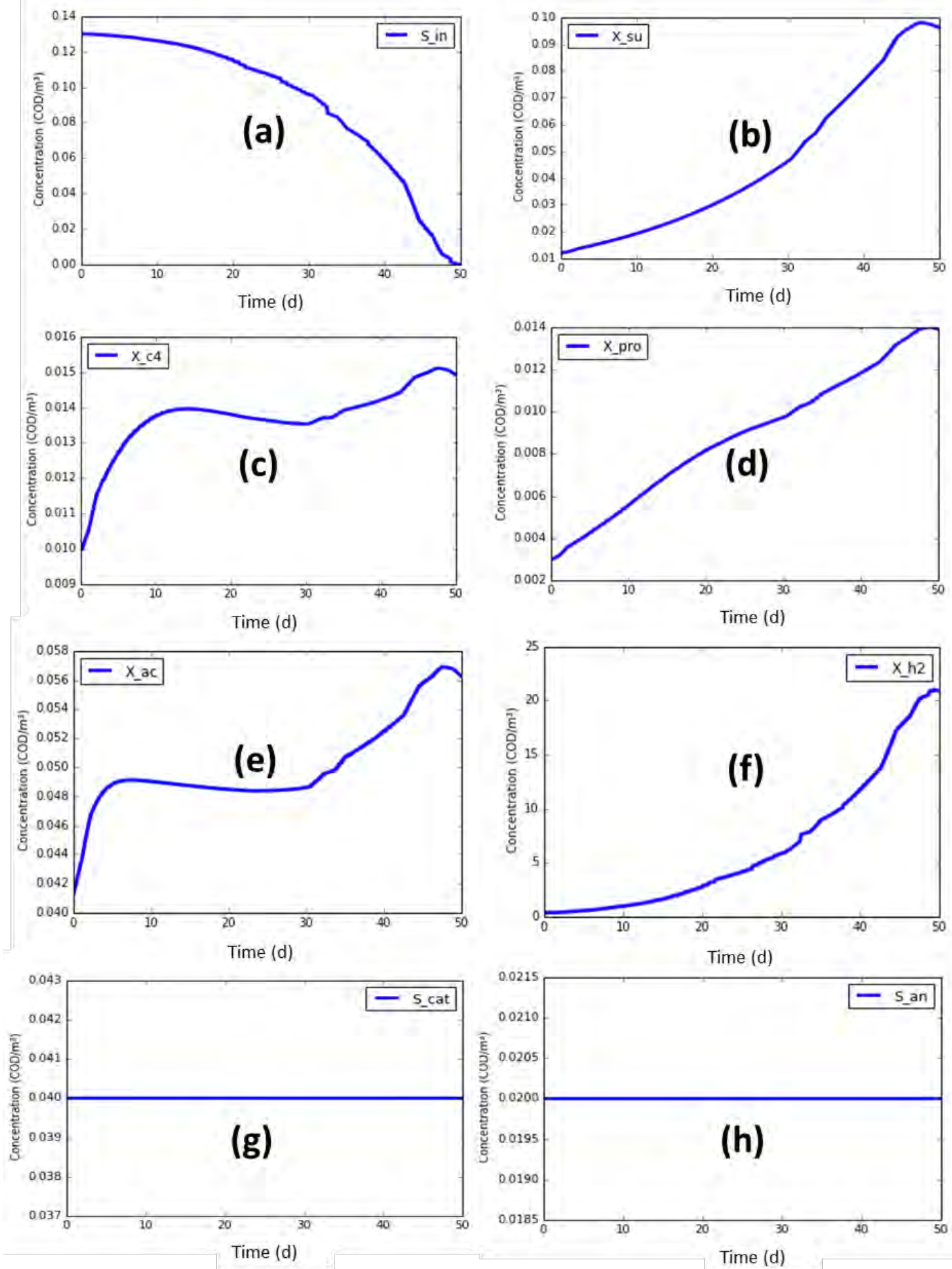

Fonte: Própria autoria. 
Figura 20 - Perfil de concentração das espécies solúveis: ácido valérico(a), ácido butírico (b), ácido propiônico (c), ácido acético (d), bicarbonato (e), amônia (f), gás hidrogênio (g) e gás metano (h)
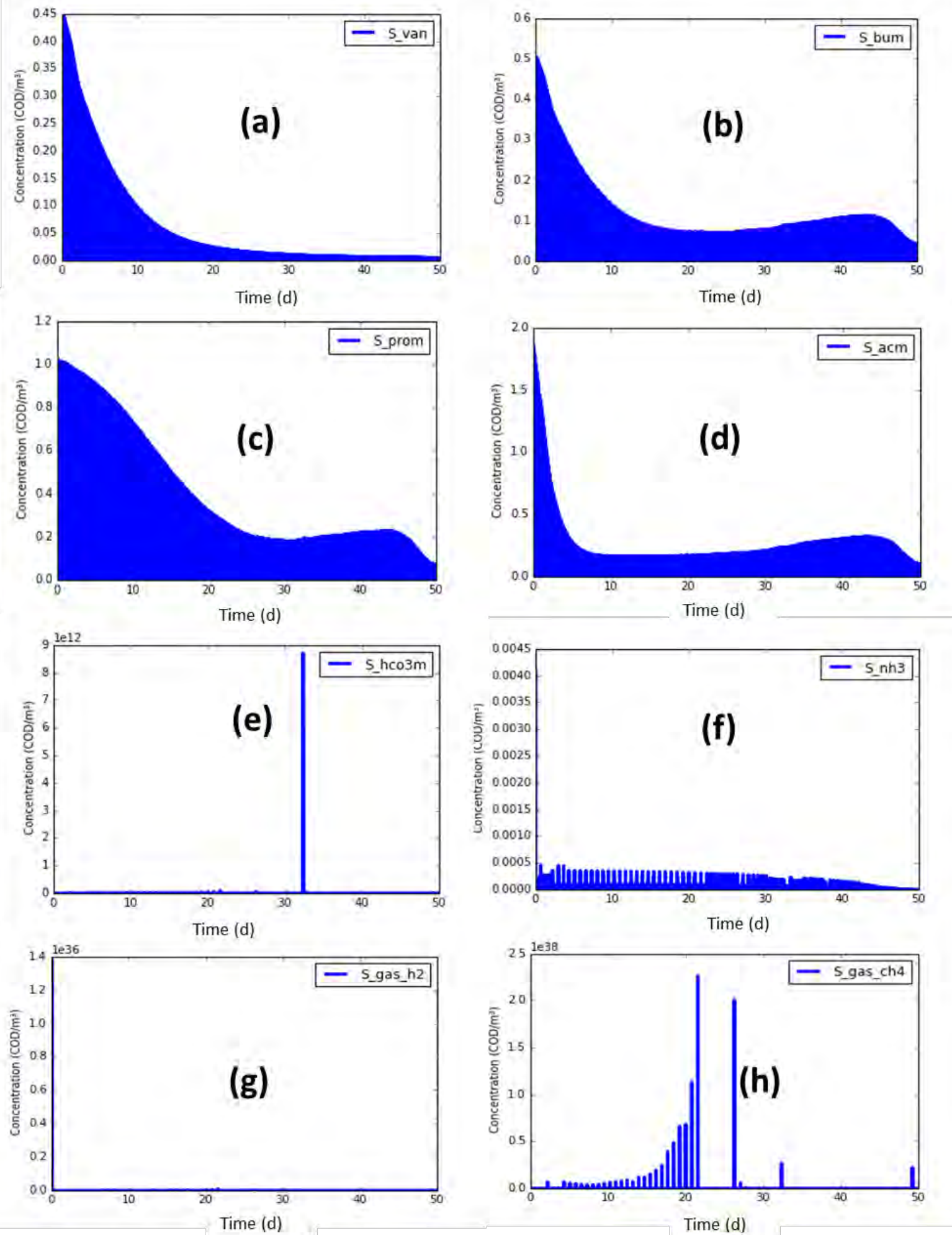

Fonte: Própria autoria. 
Figura 21 - Perfil de concentração das espécies: gás $\mathrm{CO}_{2}(\mathrm{a})$, cálcio (b), inerte solúvel (c), inerte particulado (d), lactato solúvel (e), lactato do processo de fermentação (f), lactato do processo de oxidação (g), DQO total (h)
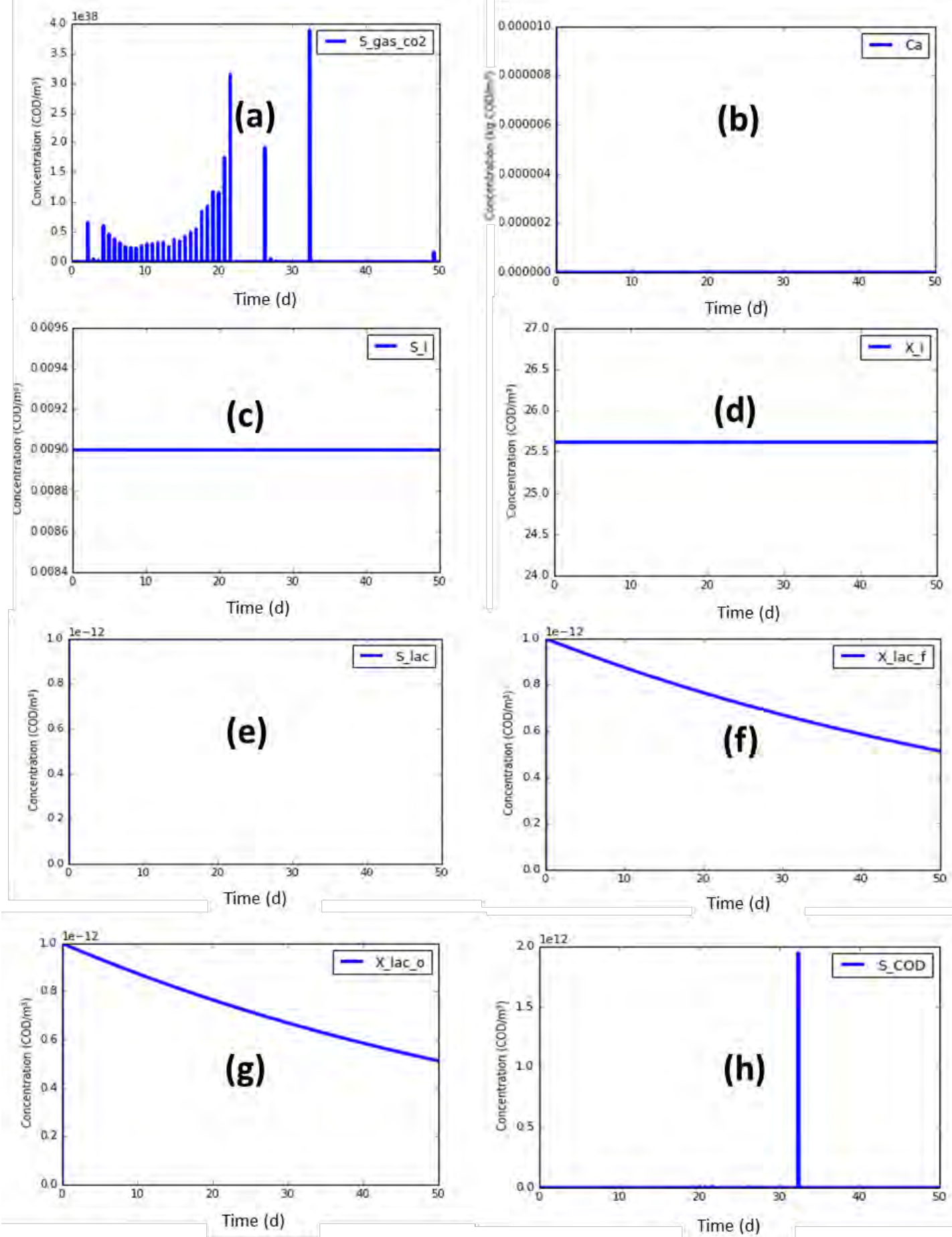

Fonte: Própria autoria. 
Figura 22 - pH calculado pela Simulação 2-A

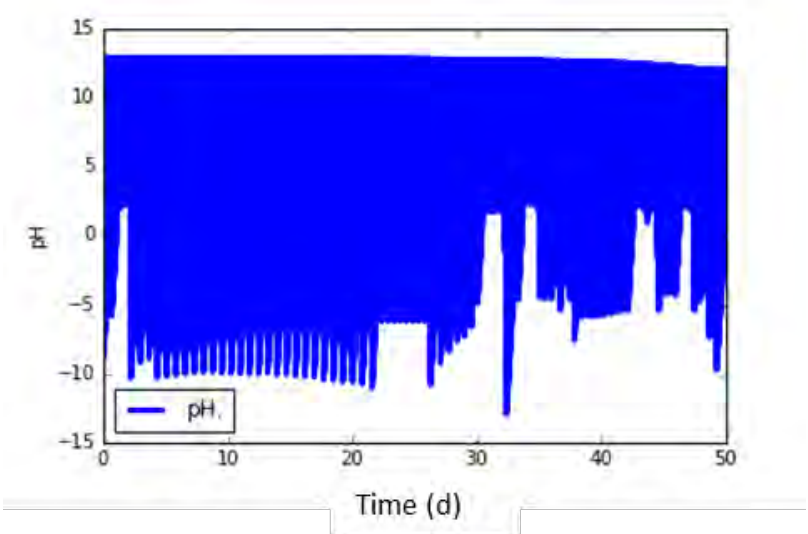

Fonte: Própria autoria.

De modo geral nesta simulação, os perfis das concentrações foram semelhantes aos encontrados por Danielsson (2014) bem como há certa similaridade com os perfis obtidos na simulação anterior. Entretanto, nesta simulação algumas espécies levaram um tempo maior para se aproximarem do estado estacionário, por exemplo açúcares solúveis, valerato, butirato e propionato, Figuras 18(a)-(d).

As concentrações de $\mathrm{H}_{2}$ e $\mathrm{CH}_{4}$ solúveis e de carbono inorgânico, Figuras 18(f)-(h), bem como bicarbonato, Figura 20(e), apresentaram instabilidades numéricas ao longo da simulação. Aproximadamente na iteração $n=518.074$ (correspondente ao instante 33 dias) houve uma instabilidade comum na simulação destas 3 espécies, na qual atingiram valores fisicamente não aceitáveis. Consequentemente, nesta iteração a DQO assumiu valores não consistentes enquanto que as concentrações de $\mathrm{H}_{2}, \mathrm{CH}_{4}$ e $\mathrm{CO}_{2}$ também divergiram.

As concentrações dos inertes solúvel e particulado, Figuras 21(c)-(d), não variaram ao longo do tempo de 50 dias, diferentemente do observado nas simulações anteriores.

\subsection{Simulação 2-B: Comparação de diferentes números de iteração}

Foram realizadas simulações com diferentes números de iterações para o tempo de 50 dias (de tratamento), para fins de comparação da influência do passo de tempo $\Delta t$ nos perfis de concentração de cada espécie. Os resultados estão apresentados nas Tabelas 21 a 25. 
Tabela 21 - Simulação feita com 80 iterações (continua)

\begin{tabular}{|c|c|c|c|c|c|c|c|c|c|c|c|c|c|c|c|c|}
\hline Iteração & ${ }_{S \text { su }}$ & $S_{\mathrm{va}}$ & $s_{\mathrm{bu}}$ & $S_{\text {pro }}$ & $s_{\mathrm{ac}}$ & $s_{\mathrm{h} 2}$ & $S_{\mathrm{ch} 4}$ & ${ }^{S} \mathrm{IC}$ & SIN & ${ }^{X_{\mathrm{su}}}$ & ${ }^{x_{\mathrm{c} 4}}$ & ${ }^{X_{\mathrm{pr}}}$ & $x_{\mathrm{ac}}$ & ${ }^{x_{\mathrm{h} 2}}$ & $s_{\text {cat }}$ & $S_{\text {an }}$ \\
\hline 0 & 1,15 & 0,052 & 0,053 & 0,123 & 0,171 & $1 \mathrm{E}-09$ & 0,000001 & 0,152678 & 0,13023 & 0,012016 & 0,01 & 0,003 & 0,04126 & 0,31702 & 0,04 & 0,02 \\
\hline 1 & 1,15 & 052 & 0,053 & 0,123 & 0,171 & 4,36E-09 & $9,89 \mathrm{E}-07$ & 152522 & 0,13023 & 0,012016 & 0,01 & 0,003 & 0,04126 & 0,317019 & 0,04 & 0,02 \\
\hline 2 & 1,15 & 0,052 & 0,053 & 0,123 & 0,171 & 4,36E-09 & $9,89 \mathrm{E}-07$ & 0,152522 & 0,13023 & 0,012016 & 0,01 & 0,003 & 0,04126 & 0,317019 & 0,04 & 0,02 \\
\hline 3 & 1,137087 & 0,039242 & 0,041309 & 0,119197 & 0,086939 & 4983362 & 7841100 & $2,52 \mathrm{E}+08$ & 0,130201 & 0,013157 & 0,011433 & 0,003499 & 0,046235 & 0,313316 & 0,04 & 0,02 \\
\hline 4 & 1,137087 & 0,039242 & 0,041309 & 0,119197 & 0,086939 & 4915212 & 7733870 & $2,48 E+08$ & 0,130201 & 0,013157 & 0,011433 & 0,003499 & 0,046235 & 0,313315 & 0,04 & 0,02 \\
\hline 5 & 1,137087 & 0,039242 & 0,041309 & 0,119197 & 0,086939 & 4915212 & 7733870 & $2,48 \mathrm{E}+08$ & 0,130201 & 0,013157 & 0,011433 & 0,003499 & 0,046235 & 0,313315 & 0,04 & 0,02 \\
\hline 6 & 1,122955 & 0,039242 & 0,042963 & 0,122631 & 0,007395 & $8,96 \mathrm{E}+09$ & $2,27 \mathrm{E}+10$ & $1,77 \mathrm{E}+13$ & 0,127835 & 0,014406 & 0,01129 & 0,003455 & 0,049895 & 0,720309 & 0,04 & 0,02 \\
\hline 7 & 1,122955 & 0,039242 & 0,042963 & 0,122631 & 0,007395 & $8,88 \mathrm{E}+09$ & $2,25 \mathrm{E}+10$ & $1,76 \mathrm{E}+13$ & 0,127835 & 0,014406 & 0,01129 & 0,003455 & 0,049895 & 0,720309 & 0,04 & 0,02 \\
\hline 8 & 1,122955 & 0,039242 & 0,042963 & 0,122631 & 0,007395 & $8,88 \mathrm{E}+09$ & $2,25 \mathrm{E}+10$ & $1,76 \mathrm{E}+13$ & 0,127835 & 0,014406 & 0,01129 & 0,003455 & 0,049895 & 0,720309 & 0,04 & 0,02 \\
\hline 9 & 1,107492 & 0,039242 & 0,044772 & 0,126388 & 0,001389 & $1 \mathrm{E}-12$ & $1 \mathrm{E}-12$ & $1 \mathrm{E}-12$ & 0,122455 & 0,015772 & 0,011149 & 0,003412 & 0,049857 & 1,655971 & 0,04 & 0,02 \\
\hline 10 & 1,107492 & 0,039242 & 0,044772 & 0,126388 & 0,001389 & $5,11 \mathrm{E}-11$ & $2,01 \mathrm{E}-10$ & $5,42 \mathrm{E}+14$ & 0,122455 & 0,015772 & 0,011149 & 0,003412 & 0,049857 & 1,655969 & 0,04 & 0,02 \\
\hline 11 & 1,107492 & 0,039242 & 0,044772 & 0,126388 & 0,001389 & $5,11 \mathrm{E}-11$ & $2,01 \mathrm{E}-10$ & $5,42 \mathrm{E}+14$ & 0,122455 & 0,015772 & 0,011149 & 0,003412 & 0,049857 & 1,655969 & 0,04 & 0,02 \\
\hline 12 & 1,10749 & 0,03924 & 0,044771 & 0,126388 & 0,001392 & $1,36 \mathrm{E}-06$ & $2,52 \mathrm{E}-07$ & $5,35 E+14$ & 0,122455 & 0,015772 & 0,011149 & 0,003412 & 0,049857 & 1,655967 & 0,04 & 0,02 \\
\hline 13 & 1,10749 & 0,03924 & 0,044771 & 0,126388 & 0,001392 & $1,36 \mathrm{E}-06$ & $2,52 \mathrm{E}-07$ & $5,35 \mathrm{E}+14$ & 0,122455 & 0,015772 & 0,011149 & 0,003412 & 0,049857 & 1,655967 & 0,04 & 0,02 \\
\hline 14 & 1,10749 & 0,03924 & 0,044771 & 0,126388 & 0,001392 & 0,0223 & 0,08966 & $1,1 \mathrm{E}+18$ & 0,122521 & 0,015575 & 0,011009 & 0,00337 & 0,049233 & 1,635267 & 0,04 & 0,02 \\
\hline 15 & 1,107488 & 0,03924 & 0,044771 & 0,126388 & 0,001392 & 0,018291 & 0,09199 & $1,09 \mathrm{E}+18$ & 0,12252 & 0,015575 & 0,011009 & 0,00337 & 0,049233 & 1,635488 & 0,04 & 0,02 \\
\hline 16 & 1,107488 & 0,03924 & 0,044771 & 0,126388 & 0,001392 & 0,018291 & 0,09199 & $1,09 \mathrm{E}+18$ & 0,12252 & 0,015575 & 0,011009 & 0,00337 & 0,049233 & 1,635488 & 0,04 & 0,02 \\
\hline 17 & 1,107488 & 0,03924 & 0,044771 & 0,126388 & 0,001392 & 0,018291 & 0,09199 & $1,09 \mathrm{E}+18$ & 0,12252 & 0,015575 & 0,011009 & 0,00337 & 0,049233 & 1,635488 & 0,04 & 0,02 \\
\hline 18 & 1,107488 & 0,03924 & 0,044771 & 0,126388 & 0,001392 & 0,018062 & 0,090838 & $1,08 \mathrm{E}+18$ & 0,12252 & 0,015575 & 0,011009 & 0,00337 & 0,049233 & 1,635486 & 0,04 & 0,02 \\
\hline 19 & 1,107488 & 0,03924 & 0,044771 & 0,126388 & 0,001392 & 0,018062 & 0,090838 & $1,08 \mathrm{E}+18$ & 0,12252 & 0,015575 & 0,011009 & 0,00337 & 0,049233 & 1,635486 & 0,04 & 0,02 \\
\hline 20 & 1,107488 & 0,03924 & 0,044771 & 0,126388 & 0,001392 & 0,018062 & 0,090838 & $1,08 \mathrm{E}+18$ & 0,12252 & 0,015575 & 0,011009 & 0,00337 & 0,049233 & 1,635486 & 0,04 & 0,02 \\
\hline 76 & 1,107487 & 0,03924 & 0,044771 & 0,126388 & 0,001393 & 0,01107 & 0,074038 & $8,75 E+17$ & 0,122519 & 0,015575 & 0,011009 & 0,00337 & 0,049232 & 1,63567 & 0,04 & 0,02 \\
\hline 77 & 1,107487 & 0,03924 & 0,044771 & 0,126388 & 0,001393 & 0,01107 & 0,074038 & $8,75 \mathrm{E}+17$ & 0,122519 & 0,015575 & 0,011009 & 0,00337 & 0,049232 & 1,63567 & 0,04 & 0,02 \\
\hline 78 & 1,107487 & 0,03924 & 0,044771 & 0,126388 & 0,001393 & 0,010927 & 0,073082 & $8,64 \mathrm{E}+17$ & 0,122519 & 0,015575 & 0,011009 & 0,00337 & 0,049232 & 1,635668 & 0,04 & 0,02 \\
\hline 79 & 1,107487 & 0,03924 & 0,044771 & 0,126388 & 0,001393 & 0,010927 & 0,073082 & $8,64 \mathrm{E}+17$ & 0,122519 & 0,015575 & 0,011009 & 0,00337 & 0,049232 & 1,635668 & 0,04 & 0,02 \\
\hline 80 & 1,107487 & 0,03924 & 0,044771 & 0,126388 & 0,001393 & 0,010927 & 0,073082 & $8,64 \mathrm{E}+17$ & 0,122519 & 0,015575 & 0,011009 & 0,00337 & 0,049232 & 1,635668 & 0,04 & 0,02 \\
\hline
\end{tabular}


Tabela 21 - Simulação feita com 80 iterações (conclusão)

\begin{tabular}{|c|c|c|c|c|c|c|c|c|c|c|c|c|c|c|c|c|}
\hline Iteração & $S_{\text {vam }}$ & ${ }^{S_{\text {bum }}}$ & $S_{\text {prom }}$ & $S_{\mathrm{acm}}$ & ${ }^{S_{\text {hco } 3 \mathrm{~m}}}$ & $S_{\mathrm{nh} 3}$ & $s_{\text {gas,h2 }}$ & ${ }^{S_{\text {gas,ch4 }}}$ & $S_{\text {gas,co2 }}$ & $S_{\mathrm{ca}}$ & ${ }^{S} \mathrm{I}$ & ${ }^{X_{\mathrm{I}}}$ & ${ }^{S}$ lac & ${ }^{X_{\text {lac, }} \mathrm{f}}$ & ${ }^{X_{\text {lac,o }}}$ & ${ }^{S} \mathrm{DQO}$ \\
\hline 0 & 0,052 & 0,053 & 0,123 & 0,171 & 0,142777 & 0,00409 & 0,00 & 0,00001 & 0,000 & 0,000 & 0,00 & 2 & 0 & 0 & 0 & 7 \\
\hline 1 & $1 \mathrm{E}-12$ & $1 \mathrm{E}-12$ & $1 \mathrm{E}-12$ & $1 \mathrm{E}-12$ & $1 \mathrm{E}-12$ & $1 \mathrm{E}-12$ & $1,73 \mathrm{E}+31$ & $1,73 \mathrm{E}+31$ & $2,38 \mathrm{E}+31$ & $1 \mathrm{E}-05$ & 0,009 & 5,61739 & $1 \mathrm{E}-12$ & $1 \mathrm{E}-12$ & $1 \mathrm{E}-12$ & $1 \mathrm{E}-12$ \\
\hline 2 & $1 \mathrm{E}-12$ & $1 \mathrm{E}-12$ & $1 \mathrm{E}-12$ & $1 \mathrm{E}-12$ & $1 \mathrm{E}-12$ & $1 \mathrm{E}-12$ & 2107095 & 2107166 & 2895940 & $1 \mathrm{E}-05$ & 0,009 & 25,61739 & $1 \mathrm{E}-12$ & $1 \mathrm{E}-12$ & $1 \mathrm{E}-12$ & $1 \mathrm{E}-12$ \\
\hline 3 & 4486,249 & 5013,672 & 10134,1 & 18572,75 & 470,6314 & 0,903707 & $1 \mathrm{E}-12$ & $1 \mathrm{E}-12$ & $1 \mathrm{E}-12$ & $1 \mathrm{E}-05$ & 0,009 & 25,61739 & $1 \mathrm{E}-12$ & $9,88 \mathrm{E}-13$ & $9,88 \mathrm{E}-13$ & 12862668 \\
\hline 4 & $1 \mathrm{E}-12$ & $1 \mathrm{E}-12$ & $1 \mathrm{E}-12$ & $1 \mathrm{E}-12$ & $1 \mathrm{E}-12$ & $1 \mathrm{E}-12$ & $3,33 \mathrm{E}+34$ & $5,24 \mathrm{E}+34$ & $1,68 \mathrm{E}+36$ & $1 \mathrm{E}-05$ & 0,009 & 25,61739 & $1 \mathrm{E}-12$ & $9,87 \mathrm{E}-13$ & $9,87 \mathrm{E}-13$ & $1 \mathrm{E}-12$ \\
\hline 5 & $1 \mathrm{E}-12$ & $1 \mathrm{E}-12$ & $1 \mathrm{E}-12$ & $1 \mathrm{E}-12$ & $1 \mathrm{E}-12$ & $1 \mathrm{E}-12$ & $4,05 \mathrm{E}+09$ & $6,37 \mathrm{E}+09$ & $2,04 \mathrm{E}+11$ & $1 \mathrm{E}-05$ & 0,009 & 25,61739 & $1 \mathrm{E}-12$ & $9,87 \mathrm{E}-13$ & $9,87 \mathrm{E}-13$ & $1 \mathrm{E}-12$ \\
\hline 6 & 3385,522 & 3907,758 & 9820,733 & 9442,629 & $7,66 \mathrm{E}+11$ & 0,903506 & $1 \mathrm{E}-12$ & $1 \mathrm{E}-12$ & $1 \mathrm{E}-12$ & $1 \mathrm{E}-05$ & 0,009 & 25,61739 & $1 \mathrm{E}-12$ & $9,75 \mathrm{E}-13$ & $9,75 \mathrm{E}-13$ & $3,17 \mathrm{E}+10$ \\
\hline 7 & $1 \mathrm{E}-12$ & $1 \mathrm{E}-12$ & $1 \mathrm{E}-12$ & $1 \mathrm{E}-12$ & $1 \mathrm{E}-12$ & $1 \mathrm{E}-12$ & $1,4 \mathrm{E}+26$ & $3,54 \mathrm{E}+26$ & $2,65 E+29$ & $1 \mathrm{E}-12$ & 0,009 & 25,61739 & $1 \mathrm{E}-12$ & $9,75 \mathrm{E}-13$ & $9,75 \mathrm{E}-13$ & $1 \mathrm{E}-12$ \\
\hline 8 & $1 \mathrm{E}-12$ & $1 \mathrm{E}-12$ & $1 \mathrm{E}-12$ & $1 \mathrm{E}-12$ & $1 \mathrm{E}-12$ & $1 \mathrm{E}-12$ & 16,96118 & 43,03139 & 32135,74 & $1 \mathrm{E}-12$ & 0,009 & 25,61739 & $1 \mathrm{E}-12$ & $9,75 \mathrm{E}-13$ & $9,75 \mathrm{E}-13$ & $1 \mathrm{E}-12$ \\
\hline 9 & 3385,522 & 4064,166 & 10103,66 & 803,1779 & $5,43 \mathrm{E}+16$ & 0,88709 & $2,65 \mathrm{E}-07$ & $6,73 \mathrm{E}-07$ & 0,000526 & $1 \mathrm{E}-12$ & 0,009 & 25,61739 & $1 \mathrm{E}-12$ & $9,63 \mathrm{E}-13$ & $9,63 \mathrm{E}-13$ & $1 \mathrm{E}-12$ \\
\hline 10 & $1 \mathrm{E}-12$ & $1 \mathrm{E}-12$ & $1 \mathrm{E}-12$ & $1 \mathrm{E}-12$ & $1 \mathrm{E}-12$ & $1 \mathrm{E}-12$ & $2,2 \mathrm{E}+21$ & $5,58 \mathrm{E}+21$ & $1 \mathrm{E}-12$ & $1 \mathrm{E}-12$ & 0,009 & 25,61739 & $1 \mathrm{E}-12$ & $9,63 \mathrm{E}-13$ & $9,63 \mathrm{E}-13$ & $1 \mathrm{E}-12$ \\
\hline 11 & $1 \mathrm{E}-12$ & $1 \mathrm{E}-12$ & $1 \mathrm{E}-12$ & $1 \mathrm{E}-12$ & $1 \mathrm{E}-12$ & $1 \mathrm{E}-12$ & $1,48 \mathrm{E}-07$ & $3,76 \mathrm{E}-07$ & $3,31 \mathrm{E}-34$ & $1 \mathrm{E}-12$ & 0,009 & 25,61739 & $1 \mathrm{E}-12$ & $9,63 \mathrm{E}-13$ & $9,63 \mathrm{E}-13$ & $1 \mathrm{E}-12$ \\
\hline 12 & 0,35233 & 0,440767 & 1,083703 & 0,015701 & $1,74 \mathrm{E}+14$ & $8,84 \mathrm{E}-05$ & $7,82 \mathrm{E}+22$ & $1,98 \mathrm{E}+23$ & $1,09 \mathrm{E}+41$ & $1 \mathrm{E}-12$ & 0,009 & 25,61739 & $1 \mathrm{E}-12$ & $9,63 \mathrm{E}-13$ & $9,63 \mathrm{E}-13$ & 1,8925 \\
\hline 13 & 0,35233 & 0,440767 & 1,083703 & 0,015701 & $1,74 \mathrm{E}+14$ & $8,84 \mathrm{E}-05$ & 0,009501 & 0,024103 & $1,32 \mathrm{E}+16$ & $1 \mathrm{E}-12$ & 0,009 & 25,61739 & $1 \mathrm{E}-12$ & $9,63 \mathrm{E}-13$ & $9,63 \mathrm{E}-13$ & 1,8925 \\
\hline 14 & $1 \mathrm{E}-12$ & $1 \mathrm{E}-12$ & $1 \mathrm{E}-12$ & $1 \mathrm{E}-12$ & $1 \mathrm{E}-12$ & $1 \mathrm{E}-12$ & $1 \mathrm{E}-12$ & $1 \mathrm{E}-12$ & $1 \mathrm{E}-12$ & $1 \mathrm{E}-12$ & 0,009 & 25,61739 & $1 \mathrm{E}-12$ & $9,51 \mathrm{E}-13$ & $9,51 \mathrm{E}-13$ & $1 \mathrm{E}-12$ \\
\hline 15 & 0,352397 & 0,440852 & 1,083935 & 0,015735 & $3,54 \mathrm{E}+17$ & $8,85 \mathrm{E}-05$ & $4,53 \mathrm{E}+24$ & $1,82 \mathrm{E}+25$ & $2,24 \mathrm{E}+44$ & $1 \mathrm{E}-12$ & 0,009 & 25,61739 & $1 \mathrm{E}-12$ & $9,51 \mathrm{E}-13$ & $9,51 \mathrm{E}-13$ & 1,891238 \\
\hline 16 & 0,352397 & 0,440852 & 1,083935 & 0,015735 & $3,54 \mathrm{E}+17$ & $8,85 \mathrm{E}-05$ & 0,550872 & 2,214815 & $2,72 \mathrm{E}+19$ & $1 \mathrm{E}-12$ & 0,009 & 25,61739 & $1 \mathrm{E}-12$ & $9,51 \mathrm{E}-13$ & $9,51 \mathrm{E}-13$ & 1,891238 \\
\hline 17 & 0,352396 & 0,440851 & 1,083932 & 0,015735 & $3,54 \mathrm{E}+17$ & $8,85 \mathrm{E}-05$ & $3,28 \mathrm{E}-49$ & $1,08 \mathrm{E}-48$ & $1 \mathrm{E}-12$ & $1 \mathrm{E}-12$ & 0,009 & 25,61739 & $1 \mathrm{E}-12$ & $9,51 \mathrm{E}-13$ & $9,51 \mathrm{E}-13$ & 1,891233 \\
\hline 18 & $1 \mathrm{E}-12$ & $1 \mathrm{E}-12$ & $1 \mathrm{E}-12$ & $1 \mathrm{E}-12$ & $1 \mathrm{E}-12$ & $1 \mathrm{E}-12$ & $2,93 \mathrm{E}+23$ & $1,47 \mathrm{E}+24$ & $1,17 \mathrm{E}+43$ & $1 \mathrm{E}-12$ & 0,009 & 25,61739 & $1 \mathrm{E}-12$ & $9,51 \mathrm{E}-13$ & $9,51 \mathrm{E}-13$ & $1 \mathrm{E}-12$ \\
\hline 19 & $1 \mathrm{E}-12$ & $1 \mathrm{E}-12$ & $1 \mathrm{E}-12$ & $1 \mathrm{E}-12$ & $1 \mathrm{E}-12$ & $1 \mathrm{E}-12$ & 0,035545 & 0,17876 & $1,43 \mathrm{E}+18$ & $1 \mathrm{E}-12$ & 0,009 & 25,61739 & $1 \mathrm{E}-12$ & $9,51 \mathrm{E}-13$ & $9,51 \mathrm{E}-13$ & $1 \mathrm{E}-12$ \\
\hline 20 & $1,01 \mathrm{E}-12$ & $1,01 \mathrm{E}-12$ & $1,02 \mathrm{E}-12$ & $1 \mathrm{E}-12$ & 7504,151 & $1 \mathrm{E}-12$ & $2,64 \mathrm{E}-46$ & $1,3 \mathrm{E}-45$ & $1,32 \mathrm{E}-27$ & $1 \mathrm{E}-12$ & 0,009 & 25,61739 & $1 \mathrm{E}-12$ & $9,51 \mathrm{E}-13$ & $9,51 \mathrm{E}-13$ & $1,04 \mathrm{E}-12$ \\
\hline 76 & $1 \mathrm{E}-12$ & $1 \mathrm{E}-12$ & $1 \mathrm{E}-12$ & $1 \mathrm{E}-12$ & $1 \mathrm{E}-12$ & $1 \mathrm{E}-12$ & 0,003668 & 0,02453 & $1,97 \mathrm{E}+17$ & $1 \mathrm{E}-12$ & 0,009 & 25,61739 & $1 \mathrm{E}-12$ & $9,51 \mathrm{E}-13$ & $9,51 \mathrm{E}-13$ & $1 \mathrm{E}-12$ \\
\hline 77 & $4,28 \mathrm{E}-05$ & $5,35 \mathrm{E}-05$ & 0,000132 & $1,91 \mathrm{E}-06$ & $3,41 \mathrm{E}+13$ & $1,07 \mathrm{E}-08$ & $8,72 \mathrm{E}-45$ & $5,81 \mathrm{E}-44$ & $5,85 \mathrm{E}-25$ & $1 \mathrm{E}-12$ & 0,009 & 25,61739 & $1 \mathrm{E}-12$ & $9,51 \mathrm{E}-13$ & $9,51 \mathrm{E}-13$ & 0,00023 \\
\hline 78 & $1 \mathrm{E}-12$ & $1 \mathrm{E}-12$ & $1 \mathrm{E}-12$ & $1 \mathrm{E}-12$ & $1 \mathrm{E}-12$ & $1 \mathrm{E}-12$ & $1,35 \mathrm{E}+24$ & $9,05 \mathrm{E}+24$ & $1,07 \mathrm{E}+44$ & $1 \mathrm{E}-12$ & 0,009 & 25,61739 & $1 \mathrm{E}-12$ & $9,51 \mathrm{E}-13$ & $9,51 \mathrm{E}-13$ & $1 \mathrm{E}-12$ \\
\hline 79 & $1 \mathrm{E}-12$ & $1 \mathrm{E}-12$ & $1 \mathrm{E}-12$ & $1 \mathrm{E}-12$ & $1 \mathrm{E}-12$ & $1 \mathrm{E}-12$ & 0,164448 & 1,099882 & $1,3 \mathrm{E}+19$ & $1 \mathrm{E}-12$ & 0,009 & 25,61739 & $1 \mathrm{E}-12$ & $9,51 \mathrm{E}-13$ & $9,51 \mathrm{E}-13$ & $1 \mathrm{E}-12$ \\
\hline 80 & $1 \mathrm{E}-12$ & $1 \mathrm{E}-12$ & $1 \mathrm{E}-12$ & $1 \mathrm{E}-12$ & 1,39E-08 & $1 \mathrm{E}-12$ & $1,43 \mathrm{E}-48$ & $7,36 \mathrm{E}-48$ & $1 \mathrm{E}-12$ & $1 \mathrm{E}-12$ & 0,009 & 25,61739 & $1 \mathrm{E}-12$ & $9,51 \mathrm{E}-13$ & $9,51 \mathrm{E}-13$ & $1 \mathrm{E}-12$ \\
\hline
\end{tabular}

Fonte: Própria autoria. 
Tabela 22 - Simulação feita com 800 iterações (continua)

\begin{tabular}{|c|c|c|c|c|c|c|c|c|c|c|c|c|c|c|c|c|}
\hline Iteração & $S_{\mathrm{su}}$ & $s_{\mathrm{va}}$ & $S_{\mathrm{bu}}$ & $S_{\text {pro }}$ & $S_{\mathrm{ac}}$ & ${ }^{s} \mathrm{~h} 2$ & $s_{\operatorname{ch} 4}$ & ${ }^{S} \mathrm{IC}$ & ${ }^{S}$ IN & $x_{\mathrm{Su}}$ & ${ }^{x_{\mathrm{c} 4}}$ & ${ }^{X_{\mathrm{pr}}}$ & $x_{\mathrm{ac}}$ & ${ }^{x_{\mathrm{h} 2}}$ & $S_{\text {cat }}$ & $S_{\text {an }}$ \\
\hline 0 & 1,15 & 0,052 & 0,053 & 0,123 & 0,171 & $1 \mathrm{E}-09$ & 0,000001 & 0,152678 & 0,13023 & 0,012016 & 0,01 & 0,003 & 0,04126 & 0,31702 & 0,04 & 0,02 \\
\hline 1 & 1,15 & 0,052 & 0,053 & 0,123 & 0,171 & 4,36E-09 & $9,89 \mathrm{E}-07$ & 0,152522 & 0,13023 & 0,012016 & 0,01 & 0,003 & 0,04126 & 0,317019 & 0,04 & 0,02 \\
\hline 2 & 1,15 & 0,052 & 0,053 & 0,123 & 0,171 & 4,36E-09 & $9,89 \mathrm{E}-07$ & 0,152522 & 0,13023 & 0,012016 & 0,01 & 0,003 & 0,04126 & 0,317019 & 0,04 & 0,02 \\
\hline 3 & 1,148709 & 0,050724 & 0,051831 & 0,12262 & 0,162594 & 498334,9 & 784108 & 25177561 & 0,130227 & 0,01213 & 0,010143 & 0,00305 & 0,041757 & 0,316649 & 0,04 & 0,02 \\
\hline 4 & 1,148709 & 0,050724 & 0,051831 & 0,12262 & 0,162594 & 491461,7 & 773293,3 & 24830304 & 0,130227 & 0,01213 & 0,010143 & 0,00305 & 0,041757 & 0,316649 & 0,04 & 0,02 \\
\hline 5 & 1,148709 & 0,050724 & 0,051831 & 0,12262 & 0,162594 & 491461,7 & 773293,3 & 24830304 & 0,130227 & 0,01213 & 0,010143 & 0,00305 & 0,041757 & 0,316649 & 0,04 & 0,02 \\
\hline 6 & 1,147405 & 0,050724 & 0,051983 & 0,122936 & 0,152223 & $1,71 \mathrm{E}+08$ & $4,29 \mathrm{E}+08$ & $3,29 \mathrm{E}+11$ & 0,129987 & 0,012245 & 0,010131 & 0,003046 & 0,042248 & 0,357781 & 0,04 & 0,02 \\
\hline 7 & 1,147405 & 0,050724 & 0,051983 & 0,122936 & 0,152223 & $1,7 \mathrm{E}+08$ & $4,25 \mathrm{E}+08$ & $3,25 \mathrm{E}+11$ & 0,129987 & 0,012245 & 0,010131 & 0,003046 & 0,042248 & 0,357781 & 0,04 & 0,02 \\
\hline 8 & 1,147405 & 0,050724 & 0,051983 & 0,122936 & 0,152223 & $1,7 \mathrm{E}+08$ & $4,25 \mathrm{E}+08$ & $3,25 \mathrm{E}+11$ & 0,129987 & 0,012245 & 0,010131 & 0,003046 & 0,042248 & 0,357781 & 0,04 & 0,02 \\
\hline 9 & 1,146089 & 0,050724 & 0,052137 & 0,123256 & 0,142077 & $1 \mathrm{E}-12$ & $1 \mathrm{E}-12$ & $1 \mathrm{E}-12$ & 0,129717 & 0,012362 & 0,010118 & 0,003042 & 0,042726 & 0,404256 & 0,04 & 0,02 \\
\hline 10 & 1,146089 & 0,050724 & 0,052137 & 0,123256 & 0,142077 & $9,88 \mathrm{E}-13$ & $9,88 \mathrm{E}-13$ & $1,25 \mathrm{E}+12$ & 0,129717 & 0,012362 & 0,010118 & 0,003042 & 0,042726 & 0,404256 & 0,04 & 0,02 \\
\hline 11 & 1,144761 & 0,049463 & 0,050968 & 0,122855 & 0,134293 & $1,58 \mathrm{E}+15$ & $6,21 \mathrm{E}+15$ & $1 \mathrm{E}-12$ & 0,129715 & 0,012479 & 0,01026 & 0,003093 & 0,043192 & 0,40375 & 0,04 & 0,02 \\
\hline 12 & 1,144761 & 0,049463 & 0,050968 & 0,122855 & 0,134293 & $1,56 \mathrm{E}+15$ & $6,13 \mathrm{E}+15$ & $4,92 \mathrm{E}+12$ & 0,129715 & 0,012479 & 0,01026 & 0,003093 & 0,043192 & 0,40375 & 0,04 & 0,02 \\
\hline 13 & 1,144761 & 0,049463 & 0,050968 & 0,122855 & 0,134293 & $1,56 \mathrm{E}+15$ & $6,13 \mathrm{E}+15$ & $4,92 \mathrm{E}+12$ & 0,129715 & 0,012479 & 0,01026 & 0,003093 & 0,043192 & 0,40375 & 0,04 & 0,02 \\
\hline 14 & 1,14342 & 0,049463 & 0,051125 & 0,123181 & 0,124594 & $1 \mathrm{E}-12$ & $1 \mathrm{E}-12$ & $1 \mathrm{E}-12$ & 0,12941 & 0,012597 & 0,010247 & 0,003089 & 0,043648 & 0,456196 & 0,04 & 0,02 \\
\hline 15 & 1,14342 & 0,049463 & 0,051125 & 0,123181 & 0,124594 & $9,88 \mathrm{E}-13$ & $9,88 \mathrm{E}-13$ & $1,88 \mathrm{E}+13$ & 0,12941 & 0,012597 & 0,010247 & 0,003089 & 0,043648 & 0,456196 & 0,04 & 0,02 \\
\hline 16 & 1,142067 & 0,048215 & 0,049959 & 0,122759 & 0,117316 & $1,94 \mathrm{E}+12$ & $3,05 \mathrm{E}+12$ & $1 \mathrm{E}-12$ & 0,129408 & 0,012717 & 0,010389 & 0,003141 & 0,044088 & 0,455626 & 0,04 & 0,02 \\
\hline 17 & 1,142067 & 0,048215 & 0,049959 & 0,122759 & 0,117316 & $1,91 \mathrm{E}+12$ & $3,01 \mathrm{E}+12$ & $7,37 \mathrm{E}+13$ & 0,129408 & 0,012717 & 0,010389 & 0,003141 & 0,044088 & 0,455625 & 0,04 & 0,02 \\
\hline 18 & 1,142067 & 0,048215 & 0,049959 & 0,122759 & 0,117316 & $1,91 \mathrm{E}+12$ & $3,01 \mathrm{E}+12$ & $7,37 \mathrm{E}+13$ & 0,129408 & 0,012717 & 0,010389 & 0,003141 & 0,044088 & 0,455625 & 0,04 & 0,02 \\
\hline 19 & 1,140701 & 0,048215 & 0,050119 & 0,123091 & 0,108153 & $1 \mathrm{E}-12$ & $1 \mathrm{E}-12$ & $1 \mathrm{E}-12$ & 0,129065 & 0,012838 & 0,010376 & 0,003137 & 0,044516 & 0,51481 & 0,04 & 0,02 \\
\hline 20 & 1,140701 & 0,048215 & 0,050119 & 0,123091 & 0,108153 & $9,88 \mathrm{E}-13$ & $9,88 \mathrm{E}-13$ & $2,81 \mathrm{E}+14$ & 0,129065 & 0,012838 & 0,010376 & 0,003137 & 0,044516 & 0,51481 & 0,04 & 0,02 \\
\hline 796 & 1,111741 & 0,036352 & 0,040114 & 0,120778 & 0,02248 & $1 \mathrm{E}-12$ & 0,020668 & $7,49 \mathrm{E}+15$ & 0,123169 & 0,015358 & 0,011598 & 0,003661 & 0,049169 & 1,526086 & 0,04 & 0,02 \\
\hline 797 & 1,11174 & 0,036351 & 0,040113 & 0,120777 & 0,02248 & $1,41 \mathrm{E}-06$ & 0,0204 & $7,39 \mathrm{E}+15$ & 0,123169 & 0,015358 & 0,011599 & 0,003661 & 0,049169 & 1,526084 & 0,04 & 0,02 \\
\hline 798 & 1,11174 & 0,036351 & 0,040113 & 0,120777 & 0,02248 & $1,41 \mathrm{E}-06$ & 0,0204 & $7,39 \mathrm{E}+15$ & 0,123169 & 0,015358 & 0,011599 & 0,003661 & 0,049169 & 1,526084 & 0,04 & 0,02 \\
\hline 799 & 1,11174 & 0,036351 & 0,040113 & 0,120777 & 0,02248 & $1,41 \mathrm{E}-06$ & 0,0204 & $7,39 \mathrm{E}+15$ & 0,123169 & 0,015358 & 0,011599 & 0,003661 & 0,049169 & 1,526084 & 0,04 & 0,02 \\
\hline 800 & 1,111738 & 0,03635 & 0,040112 & 0,120777 & 0,022479 & $1 \mathrm{E}-12$ & 0,020688 & $7,29 \mathrm{E}+15$ & 0,123169 & 0,015358 & 0,011599 & 0,003661 & 0,049169 & 1,526117 & 0,04 & 0,02 \\
\hline
\end{tabular}


Tabela 22 - Simulação feita com 800 iterações (conclusão)

\begin{tabular}{|c|c|c|c|c|c|c|c|c|c|c|c|c|c|c|c|c|}
\hline Iteração & $S_{\text {vam }}$ & ${ }^{s}$ bum & $S_{\text {prom }}$ & $S_{\mathrm{acm}}$ & ${ }^{s}$ hco3m & $S_{\text {nh3 }}$ & $s_{\text {gas, h2 }}$ & $s_{\text {gas,ch4 }}$ & $S_{\text {gas,co } 2}$ & $S_{\mathrm{ca}}$ & ${ }^{S} \mathrm{I}$ & ${ }^{x} \mathrm{I}$ & ${ }^{S}$ lac & ${ }^{X}$ lac,f & ${ }^{X}$ lac,o & ${ }^{S} \mathrm{DQO}$ \\
\hline 0 & 0,052 & 0,053 & 0,123 & 0,171 & 0,142777 & 0,00409 & 0,00001 & 0,00001 & 0,00001 & 0,00001 & 0,009 & 25,61739 & 0 & 0 & 0 & 2,7 \\
\hline 1 & $1 \mathrm{E}-12$ & $1 \mathrm{E}-12$ & $1 \mathrm{E}-12$ & $1 \mathrm{E}-12$ & $1 \mathrm{E}-12$ & $1 \mathrm{E}-12$ & $1,73 \mathrm{E}+31$ & $1,73 \mathrm{E}+31$ & $2,38 \mathrm{E}+31$ & $1 \mathrm{E}-05$ & 0,009 & 25,61739 & $1 \mathrm{E}-12$ & $1 \mathrm{E}-12$ & $1 \mathrm{E}-12$ & $1 \mathrm{E}-12$ \\
\hline 2 & $1 \mathrm{E}-12$ & $1 \mathrm{E}-12$ & $1 \mathrm{E}-12$ & $1 \mathrm{E}-12$ & 12 & $1 \mathrm{E}-12$ & 2107090 & 2107161 & 2895933 & $1 \mathrm{E}-05$ & 0,009 & 5,61739 & $1 \mathrm{E}-12$ & $1 \mathrm{E}-12$ & $1 \mathrm{E}-12$ & $1 \mathrm{E}-12$ \\
\hline 3 & 448,6249 & 501,3672 & 1013,41 & 1857,275 & 47,06314 & 0,090371 & $1 \mathrm{E}-12$ & 2 & $1 \mathrm{E}-12$ & $1 \mathrm{E}-05$ & 0,009 & 25,61739 & $1 \mathrm{E}-12$ & $9,99 \mathrm{E}-13$ & $9,99 \mathrm{E}-13$ & 1286264 \\
\hline 4 & $1 \mathrm{E}-12$ & $1 \mathrm{E}-12$ & $1 \mathrm{E}-12$ & $1 \mathrm{E}-12$ & $1 \mathrm{E}-12$ & $1 \mathrm{E}-12$ & $6,16 \mathrm{E}+33$ & $9,7 \mathrm{E}+33$ & $3,11 \mathrm{E}+35$ & $1 \mathrm{E}-05$ & 0,009 & 25,61739 & $1 \mathrm{E}-12$ & $9,99 \mathrm{E}-13$ & $9,99 \mathrm{E}-13$ & $1 \mathrm{E}-12$ \\
\hline 5 & $1 \mathrm{E}-12$ & $1 \mathrm{E}-12$ & $1 \mathrm{E}-12$ & $1 \mathrm{E}-12$ & $1 \mathrm{E}-12$ & $1 \mathrm{E}-12$ & $7,49 \mathrm{E}+08$ & $1,18 \mathrm{E}+09$ & $3,78 \mathrm{E}+10$ & $1 \mathrm{E}-05$ & 0,009 & 25,61739 & $1 \mathrm{E}-12$ & $9,99 \mathrm{E}-13$ & $9,99 \mathrm{E}-13$ & $1 \mathrm{E}-12$ \\
\hline 6 & 437,6176 & 490,308 & 1010,276 & 1765,973 & $7,66 \mathrm{E}+09$ & 0,090369 & $1 \mathrm{E}-12$ & $1 \mathrm{E}-12$ & $1 \mathrm{E}-12$ & $1 \mathrm{E}-05$ & 0,009 & 25,61739 & $1 \mathrm{E}-12$ & $9,98 \mathrm{E}-13$ & $9,98 \mathrm{E}-13$ & $6 \mathrm{E}+08$ \\
\hline 7 & $1 \mathrm{E}-12$ & $1 \mathrm{E}-12$ & $1 \mathrm{E}-12$ & $1 \mathrm{E}-12$ & $1 \mathrm{E}-12$ & $1 \mathrm{E}-12$ & $1,99 \mathrm{E}+28$ & $4,99 \mathrm{E}+28$ & $3,73 \mathrm{E}+31$ & $1 \mathrm{E}-12$ & 0,009 & 25,61739 & $1 \mathrm{E}-12$ & $9,97 \mathrm{E}-13$ & $9,97 \mathrm{E}-13$ & $1 \mathrm{E}-12$ \\
\hline 8 & $1 \mathrm{E}-12$ & $1 \mathrm{E}-12$ & $1 \mathrm{E}-12$ & $1 \mathrm{E}-12$ & $1 \mathrm{E}-12$ & $1 \mathrm{E}-12$ & 2421,526 & 6066,964 & 4533485 & $1 \mathrm{E}-12$ & 0,009 & 25,61739 & $1 \mathrm{E}-12$ & $9,97 \mathrm{E}-13$ & $9,97 \mathrm{E}-13$ & $1 \mathrm{E}-12$ \\
\hline 9 & 437,6176 & 491,7507 & 1012,886 & 1653,336 & $1 \mathrm{E}+14$ & 0,090202 & $2,55 \mathrm{E}-13$ & $6,38 \mathrm{E}-13$ & $4,88 \mathrm{E}-10$ & $1 \mathrm{E}-12$ & 0,009 & 25,61739 & $1 \mathrm{E}-12$ & $9,96 \mathrm{E}-13$ & $9,96 \mathrm{E}-13$ & $1 \mathrm{E}-12$ \\
\hline 10 & $1 \mathrm{E}-12$ & $1 \mathrm{E}-12$ & $1 \mathrm{E}-12$ & $1 \mathrm{E}-12$ & $1 \mathrm{E}-12$ & $1 \mathrm{E}-12$ & $6,67 \mathrm{E}+15$ & $1,67 \mathrm{E}+16$ & $1 \mathrm{E}-12$ & $1 \mathrm{E}-12$ & 0,009 & 25,61739 & $1 \mathrm{E}-12$ & $9,96 \mathrm{E}-13$ & $9,96 \mathrm{E}-13$ & $1 \mathrm{E}-12$ \\
\hline 11 & 437,6176 & 493,207 & 1015,52 & 1543,138 & $3,85 \mathrm{E}+14$ & 0,090015 & $1 \mathrm{E}-12$ & $1 \mathrm{E}-12$ & $8,39 \mathrm{E}-26$ & $1 \mathrm{E}-12$ & 0,009 & 25,61739 & $1 \mathrm{E}-12$ & $9,95 \mathrm{E}-13$ & $9,95 \mathrm{E}-13$ & $7,79 \mathrm{E}+15$ \\
\hline 12 & 1E-12 & $1 \mathrm{E}-12$ & 1E-12 & 1E-12 & $1 \mathrm{E}-12$ & $1 \mathrm{E}-12$ & $9,61 \mathrm{E}+40$ & $3,78 \mathrm{E}+41$ & 1E-12 & $1 \mathrm{E}-12$ & 0,009 & 25,61739 & 1E-12 & $9,95 \mathrm{E}-13$ & $9,95 \mathrm{E}-13$ & 1E-12 \\
\hline 13 & $1 \mathrm{E}-12$ & $1 \mathrm{E}-12$ & $1 \mathrm{E}-12$ & $1 \mathrm{E}-12$ & $1 \mathrm{E}-12$ & $1 \mathrm{E}-12$ & $1,17 \mathrm{E}+16$ & $4,6 \mathrm{E}+16$ & $1,21 \mathrm{E}-37$ & $1 \mathrm{E}-12$ & 0,009 & 25,61739 & $1 \mathrm{E}-12$ & $9,95 \mathrm{E}-13$ & $9,95 \mathrm{E}-13$ & $1 \mathrm{E}-12$ \\
\hline 14 & 426,7403 & 482,1472 & 1012,217 & 1458,593 & $1,52 \mathrm{E}+15$ & 0,090013 & $1,97 \mathrm{E}-23$ & $7,02 \mathrm{E}-23$ & $7,25 \mathrm{E}-26$ & $1 \mathrm{E}-12$ & 0,009 & 25,61739 & $1 \mathrm{E}-12$ & $9,94 \mathrm{E}-13$ & $9,94 \mathrm{E}-13$ & $1 \mathrm{E}-12$ \\
\hline 15 & $1 \mathrm{E}-12$ & $1 \mathrm{E}-12$ & $1 \mathrm{E}-12$ & $1 \mathrm{E}-12$ & $1 \mathrm{E}-12$ & $1 \mathrm{E}-12$ & $8,2 \mathrm{E}+12$ & $8,2 \mathrm{E}+12$ & $1 \mathrm{E}-12$ & $1 \mathrm{E}-12$ & 0,009 & 25,61739 & $1 \mathrm{E}-12$ & $9,94 \mathrm{E}-13$ & $9,94 \mathrm{E}-13$ & $1 \mathrm{E}-12$ \\
\hline 16 & 426,7403 & 483,6311 & 1014,902 & 1353,251 & $5,81 \mathrm{E}+15$ & 0,089802 & $1 \mathrm{E}-12$ & $1 \mathrm{E}-12$ & $1,42 \mathrm{E}-18$ & $1 \mathrm{E}-12$ & 0,009 & 25,61739 & $1 \mathrm{E}-12$ & $9,93 \mathrm{E}-13$ & $9,93 \mathrm{E}-13$ & $4,99 \mathrm{E}+12$ \\
\hline 17 & $1 \mathrm{E}-12$ & $1 \mathrm{E}-12$ & $1 \mathrm{E}-12$ & $1 \mathrm{E}-12$ & $1 \mathrm{E}-12$ & $1 \mathrm{E}-12$ & $7,03 \mathrm{E}+37$ & $1,11 \mathrm{E}+38$ & $1 \mathrm{E}-12$ & $1 \mathrm{E}-12$ & 0,009 & 25,61739 & $1 \mathrm{E}-12$ & $9,93 \mathrm{E}-13$ & $9,93 \mathrm{E}-13$ & $1 \mathrm{E}-12$ \\
\hline 18 & $1 \mathrm{E}-12$ & $1 \mathrm{E}-12$ & $1 \mathrm{E}-12$ & $1 \mathrm{E}-12$ & $1 \mathrm{E}-12$ & $1 \mathrm{E}-12$ & $8,54 \mathrm{E}+12$ & $1,34 \mathrm{E}+13$ & $1,21 \mathrm{E}-37$ & $1 \mathrm{E}-12$ & 0,009 & 25,61739 & $1 \mathrm{E}-12$ & $9,93 \mathrm{E}-13$ & $9,93 \mathrm{E}-13$ & $1 \mathrm{E}-12$ \\
\hline 19 & 415,9737 & 472,6006 & 1011,425 & 1274,2 & $2,28 \mathrm{E}+16$ & 0,089801 & $9,78 \mathrm{E}-20$ & $1,46 \mathrm{E}-19$ & $4,12 \mathrm{E}-18$ & $1 \mathrm{E}-12$ & 0,009 & 25,61739 & $1 \mathrm{E}-12$ & $9,91 \mathrm{E}-13$ & $9,91 \mathrm{E}-13$ & $1 \mathrm{E}-12$ \\
\hline 20 & $1 \mathrm{E}-12$ & $1 \mathrm{E}-12$ & $1 \mathrm{E}-12$ & $1 \mathrm{E}-12$ & $1 \mathrm{E}-12$ & $1 \mathrm{E}-12$ & $6,09 \mathrm{E}+12$ & $6,1 \mathrm{E}+12$ & $1 \mathrm{E}-12$ & $1 \mathrm{E}-12$ & 0,009 & 25,61739 & $1 \mathrm{E}-12$ & $9,91 \mathrm{E}-13$ & $9,91 \mathrm{E}-13$ & $1 \mathrm{E}-12$ \\
\hline 796 & $1 \mathrm{E}-12$ & $1 \mathrm{E}-12$ & $1 \mathrm{E}-12$ & $1 \mathrm{E}-12$ & $1 \mathrm{E}-12$ & $1 \mathrm{E}-12$ & $1 \mathrm{E}-12$ & $1 \mathrm{E}-12$ & $1 \mathrm{E}-12$ & $1 \mathrm{E}-12$ & 0,009 & 25,61739 & $1 \mathrm{E}-12$ & $9,65 \mathrm{E}-13$ & $9,65 \mathrm{E}-13$ & $1 \mathrm{E}-12$ \\
\hline 797 & 0,329587 & 0,398784 & 1,045759 & 0,256591 & $2,43 \mathrm{E}+15$ & $8,98 \mathrm{E}-05$ & $1,03 \mathrm{E}+18$ & $8,1 \mathrm{E}+24$ & $2,94 \mathrm{E}+42$ & $1 \mathrm{E}-12$ & 0,009 & 25,61739 & $1 \mathrm{E}-12$ & $9,65 \mathrm{E}-13$ & $9,65 \mathrm{E}-13$ & 2,030449 \\
\hline 798 & 0,329587 & 0,398784 & 1,045759 & 0,256591 & $2,43 \mathrm{E}+15$ & $8,98 \mathrm{E}-05$ & $1,25 \mathrm{E}-07$ & 0,984267 & $3,57 \mathrm{E}+17$ & $1 \mathrm{E}-12$ & 0,009 & 25,61739 & $1 \mathrm{E}-12$ & $9,65 \mathrm{E}-13$ & $9,65 \mathrm{E}-13$ & 2,030449 \\
\hline 799 & $1 \mathrm{E}-12$ & $1 \mathrm{E}-12$ & $1 \mathrm{E}-12$ & $1 \mathrm{E}-12$ & $1 \mathrm{E}-12$ & $1 \mathrm{E}-12$ & $3,41 \mathrm{E}-49$ & $1 \mathrm{E}-12$ & $1 \mathrm{E}-12$ & $1 \mathrm{E}-12$ & 0,009 & 25,61739 & $1 \mathrm{E}-12$ & $9,65 \mathrm{E}-13$ & $9,65 \mathrm{E}-13$ & $1 \mathrm{E}-12$ \\
\hline 800 & 0,330438 & 0,399815 & 1,048487 & 0,257257 & $2,4 \mathrm{E}+15$ & $9,01 \mathrm{E}-05$ & $6,62 \mathrm{E}+20$ & $9,58 \mathrm{E}+24$ & $3,47 \mathrm{E}+42$ & $1 \mathrm{E}-12$ & 0,009 & 25,61739 & $1 \mathrm{E}-12$ & $9,65 \mathrm{E}-13$ & $9,65 \mathrm{E}-13$ & 2,035692 \\
\hline
\end{tabular}

Fonte: Própria autoria. 
Tabela 23- Simulação feita com 8.000 iterações (continua)

\begin{tabular}{|c|c|c|c|c|c|c|c|c|c|c|c|c|c|c|c|c|}
\hline Iteração & $S_{\mathrm{su}}$ & $S_{\mathrm{va}}$ & ${ }^{S_{\mathrm{bu}}}$ & $S_{\text {pro }}$ & $S_{\mathrm{ac}}$ & ${ }^{S} \mathrm{~h} 2$ & $S_{\operatorname{ch} 4}$ & ${ }^{S_{\mathrm{IC}}}$ & ${ }^{S_{\mathrm{IN}}}$ & $X_{\mathrm{su}}$ & $x_{\mathrm{c} 4}$ & $X_{\mathrm{pr}}$ & $x_{\mathrm{ac}}$ & ${ }^{x_{\mathrm{h} 2}}$ & $S_{\text {cat }}$ & $S_{\text {an }}$ \\
\hline 0 & 1,15 & 0,052 & 0,053 & 0,123 & 0,171 & $1 \mathrm{E}-09$ & 0,000001 & 0,152678 & 0,13023 & 0,012016 & 0,01 & 0,003 & 0,04126 & 0,31702 & 0,04 & 0,02 \\
\hline 1 & 1,15 & 0,052 & 0,053 & 0,123 & 0,171 & $4,36 \mathrm{E}-09$ & 9,89E-07 & 0,152522 & 0,13023 & 0,012016 & 0,01 & 0,003 & 0,04126 & 0,317019 & 0,04 & 0,02 \\
\hline 2 & 1,15 & 0,052 & 0,053 & 0,123 & 0,171 & $4,36 \mathrm{E}-09$ & 9,89E-07 & 0,152522 & 0,13023 & 0,012016 & 0,01 & 0,003 & 0,04126 & 0,317019 & 0,04 & 0,02 \\
\hline 3 & 1,149871 & 0,051872 & 0,052883 & 0,122962 & 0,170159 & 49833,57 & 78410,93 & 2517760 & 0,13023 & 0,012027 & 0,010014 & 0,003005 & 0,04131 & 0,316982 & 0,04 & 0,02 \\
\hline 4 & 1,149871 & 0,051872 & 0,052883 & 0,122962 & 0,170159 & 49140,29 & 77320,08 & 2482733 & 0,13023 & 0,012027 & 0,010014 & 0,003005 & 0,04131 & 0,316982 & 0,04 & 0,02 \\
\hline 5 & 1,149871 & 0,051872 & 0,052883 & 0,122962 & 0,170159 & 49140,29 & 77320,08 & 2482733 & 0,13023 & 0,012027 & 0,010014 & 0,003005 & 0,04131 & 0,316982 & 0,04 & 0,02 \\
\hline 6 & 1,149742 & 0,051872 & 0,052898 & 0,122993 & 0,16911 & 3309638 & 8204689 & $6,17 \mathrm{E}+09$ & 0,130206 & 0,012039 & 0,010013 & 0,003005 & 0,041359 & 0,3211 & 0,04 & 0,02 \\
\hline 7 & 1,149742 & 0,051872 & 0,052898 & 0,122993 & 0,16911 & 3269812 & 8105960 & $6,1 \mathrm{E}+09$ & 0,130206 & 0,012039 & 0,010013 & 0,003005 & 0,041359 & 0,321099 & 0,04 & 0,02 \\
\hline 8 & 1,149742 & 0,051872 & 0,052898 & 0,122993 & 0,16911 & 3269812 & 8105960 & $6,1 \mathrm{E}+09$ & 0,130206 & 0,012039 & 0,010013 & 0,003005 & 0,041359 & 0,321099 & 0,04 & 0,02 \\
\hline 9 & 1,149612 & 0,051872 & 0,052913 & 0,123025 & 0,168063 & $1 \mathrm{E}-12$ & $1 \mathrm{E}-12$ & $1 \mathrm{E}-12$ & 0,130181 & 0,01205 & 0,010012 & 0,003004 & 0,041409 & 0,32527 & 0,04 & 0,02 \\
\hline 10 & 1,149612 & 0,051872 & 0,052913 & 0,123025 & 0,168063 & $9,87 \mathrm{E}-13$ & $9,87 \mathrm{E}-13$ & $2,4 \mathrm{E}+09$ & 0,130181 & 0,01205 & 0,010012 & 0,003004 & 0,041409 & 0,32527 & 0,04 & 0,02 \\
\hline 11 & 1,149483 & 0,051745 & 0,052796 & 0,122986 & 0,167228 & $1,25 \mathrm{E}+12$ & $1,97 \mathrm{E}+12$ & $1 \mathrm{E}-12$ & 0,130181 & 0,012062 & 0,010026 & 0,003009 & 0,041458 & 0,325229 & 0,04 & 0,02 \\
\hline 12 & 1,149483 & 0,051745 & 0,052796 & 0,122986 & 0,167228 & $1,23 \mathrm{E}+12$ & $1,94 \mathrm{E}+12$ & $9,72 \mathrm{E}+08$ & 0,130181 & 0,012062 & 0,010026 & 0,003009 & 0,041458 & 0,325229 & 0,04 & 0,02 \\
\hline 13 & 1,149483 & 0,051745 & 0,052796 & 0,122986 & 0,167228 & $1,23 \mathrm{E}+12$ & $1,94 \mathrm{E}+12$ & $9,72 \mathrm{E}+08$ & 0,130181 & 0,012062 & 0,010026 & 0,003009 & 0,041458 & 0,325229 & 0,04 & 0,02 \\
\hline 14 & 1,149353 & 0,051745 & 0,052812 & 0,123018 & 0,166183 & $1,07 \mathrm{E}+12$ & $2,94 \mathrm{E}+12$ & $1 \mathrm{E}-12$ & 0,130157 & 0,012073 & 0,010025 & 0,003009 & 0,041508 & 0,329453 & 0,04 & 0,02 \\
\hline 15 & 1,149353 & 0,051745 & 0,052812 & 0,123018 & 0,166183 & $1,06 \mathrm{E}+12$ & $2,9 \mathrm{E}+12$ & $3,91 \mathrm{E}+08$ & 0,130157 & 0,012073 & 0,010025 & 0,003009 & 0,041508 & 0,329453 & 0,04 & 0,02 \\
\hline 16 & 1,149353 & 0,051745 & 0,052812 & 0,123018 & 0,166183 & $1,06 \mathrm{E}+12$ & $2,9 \mathrm{E}+12$ & $3,91 \mathrm{E}+08$ & 0,130157 & 0,012073 & 0,010025 & 0,003009 & 0,041508 & 0,329453 & 0,04 & 0,02 \\
\hline 17 & 1,149223 & 0,051745 & 0,052827 & 0,123049 & 0,165141 & $4,6 \mathrm{E}+11$ & $2,39 \mathrm{E}+12$ & $1 \mathrm{E}-12$ & 0,130132 & 0,012085 & 0,010024 & 0,003008 & 0,041557 & 0,333733 & 0,04 & 0,02 \\
\hline 18 & 1,149223 & 0,051745 & 0,052827 & 0,123049 & 0,165141 & $4,54 \mathrm{E}+11$ & $2,36 \mathrm{E}+12$ & $1,57 \mathrm{E}+08$ & 0,130132 & 0,012085 & 0,010024 & 0,003008 & 0,041557 & 0,333732 & 0,04 & 0,02 \\
\hline 19 & 1,149223 & 0,051745 & 0,052827 & 0,123049 & 0,165141 & $4,54 \mathrm{E}+11$ & $2,36 \mathrm{E}+12$ & $1,57 \mathrm{E}+08$ & 0,130132 & 0,012085 & 0,010024 & 0,003008 & 0,041557 & 0,333732 & 0,04 & 0,02 \\
\hline 20 & 1,149094 & 0,051745 & 0,052842 & 0,123081 & 0,164101 & $1,8 \mathrm{E}+11$ & $1,81 \mathrm{E}+12$ & $1 \mathrm{E}-12$ & 0,130106 & 0,012096 & 0,010022 & 0,003008 & 0,041606 & 0,338067 & 0,04 & 0,02 \\
\hline 7.996 & 1,143564 & 0,047524 & 0,049058 & 0,122259 & 0,129323 & $1,28 \mathrm{E}-06$ & 0,002419 & $3,43 \mathrm{E}+16$ & 0,129864 & 0,012566 & 0,010473 & 0,00316 & 0,043507 & 0,376611 & 0,04 & 0,02 \\
\hline 7.997 & 1,143564 & 0,047524 & 0,049058 & 0,122259 & 0,129323 & 1,28E-06 & 0,002419 & $3,43 \mathrm{E}+16$ & 0,129864 & 0,012566 & 0,010473 & 0,00316 & 0,043507 & 0,376611 & 0,04 & 0,02 \\
\hline 7.998 & 1,143563 & 0,047523 & 0,049057 & 0,122259 & 0,129315 & $1 \mathrm{E}-12$ & 0,002522 & $3,39 \mathrm{E}+16$ & 0,129864 & 0,012566 & 0,010473 & 0,00316 & 0,043507 & 0,376619 & 0,04 & 0,02 \\
\hline 7.999 & 1,143563 & 0,047523 & 0,049057 & 0,122259 & 0,129315 & $1 \mathrm{E}-12$ & 0,002522 & $3,39 \mathrm{E}+16$ & 0,129864 & 0,012566 & 0,010473 & 0,00316 & 0,043507 & 0,376619 & 0,04 & 0,02 \\
\hline 8.000 & 1,143563 & 0,047523 & 0,049057 & 0,122259 & 0,129315 & $1 \mathrm{E}-12$ & 0,002522 & $3,39 \mathrm{E}+16$ & 0,129864 & 0,012566 & 0,010473 & 0,00316 & 0,043507 & 0,376619 & 0,04 & 0,02 \\
\hline
\end{tabular}


Tabela 23 - Simulação feita com 8.000 iterações (conclusão)

\begin{tabular}{|c|c|c|c|c|c|c|c|c|c|c|c|c|c|c|c|c|}
\hline Iteração & $S_{\text {vam }}$ & ${ }^{S}$ bum & $S_{\text {prom }}$ & $S_{\mathrm{acm}}$ & ${ }^{S_{\mathrm{hco}} 3 \mathrm{~m}}$ & $S_{\text {nh3 }}$ & $S_{\text {gas, h2 }}$ & ${ }^{S_{\text {gas,ch} 4}}$ & $S_{\text {gas,co2 }}$ & $S_{\mathrm{ca}}$ & ${ }^{S}$ I & ${ }^{X_{\mathrm{I}}}$ & ${ }^{S}$ lac & ${ }^{X_{\text {lac, }} \mathrm{f}}$ & ${ }^{X_{\text {lac,o }}}$ & ${ }^{S} \mathrm{DQO}$ \\
\hline 0 & 0,052 & 0,053 & 0,123 & 0,171 & 0,142777 & 0,00409 & 0,00001 & 0,00001 & 0,00001 & 0,00001 & 0,009 & 25,61739 & 0 & 0 & 0 & 2,7 \\
\hline 1 & $1 \mathrm{E}-12$ & $1 \mathrm{E}-12$ & $1 \mathrm{E}-12$ & $1 \mathrm{E}-12$ & $1 \mathrm{E}-12$ & $1 \mathrm{E}-12$ & $1,73 \mathrm{E}+31$ & $1,73 \mathrm{E}+31$ & $2,38 \mathrm{E}+31$ & $1 \mathrm{E}-05$ & 0,009 & 25,61739 & $1 \mathrm{E}-12$ & $1 \mathrm{E}-12$ & $1 \mathrm{E}-12$ & $1 \mathrm{E}-12$ \\
\hline 2 & $1 \mathrm{E}-12$ & $1 \mathrm{E}-12$ & $1 \mathrm{E}-12$ & $1 \mathrm{E}-12$ & $1 \mathrm{E}-12$ & $1 \mathrm{E}-12$ & 2107093 & 2107164 & 2895937 & $1 \mathrm{E}-05$ & 0,009 & 25,61739 & $1 \mathrm{E}-12$ & $1 \mathrm{E}-12$ & $1 \mathrm{E}-12$ & $1 \mathrm{E}-12$ \\
\hline 3 & 44,86249 & 50,13672 & 101,341 & 185,7275 & 4,706314 & 0,009037 & $1 \mathrm{E}-12$ & $1 \mathrm{E}-12$ & $1 \mathrm{E}-12$ & $1 \mathrm{E}-05$ & 0,009 & 25,61739 & $1 \mathrm{E}-12$ & $1 \mathrm{E}-12$ & $1 \mathrm{E}-12$ & 128626,6 \\
\hline 4 & $1 \mathrm{E}-12$ & $1 \mathrm{E}-12$ & $1 \mathrm{E}-12$ & $1 \mathrm{E}-12$ & $1 \mathrm{E}-12$ & $1 \mathrm{E}-12$ & $1,16 \mathrm{E}+33$ & $1,82 \mathrm{E}+33$ & $5,84 \mathrm{E}+34$ & $1 \mathrm{E}-05$ & 0,009 & 25,61739 & $1 \mathrm{E}-12$ & $1 \mathrm{E}-12$ & $1 \mathrm{E}-12$ & $1 \mathrm{E}-12$ \\
\hline 5 & $1 \mathrm{E}-12$ & $1 \mathrm{E}-12$ & $1 \mathrm{E}-12$ & $1 \mathrm{E}-12$ & $1 \mathrm{E}-12$ & $1 \mathrm{E}-12$ & $1,4 \mathrm{E}+08$ & $2,21 \mathrm{E}+08$ & $7,1 \mathrm{E}+09$ & $1 \mathrm{E}-05$ & 0,009 & 25,61739 & $1 \mathrm{E}-12$ & $1 \mathrm{E}-12$ & $1 \mathrm{E}-12$ & $1 \mathrm{E}-12$ \\
\hline 6 & 44,75242 & 50,02612 & 101,3097 & 184,8145 & 76608931 & 0,009037 & $1 \mathrm{E}-12$ & $1 \mathrm{E}-12$ & $1 \mathrm{E}-12$ & $1 \mathrm{E}-05$ & 0,009 & 25,61739 & $1 \mathrm{E}-12$ & $1 \mathrm{E}-12$ & $1 \mathrm{E}-12$ & 11388248 \\
\hline 7 & $1 \mathrm{E}-12$ & $1 \mathrm{E}-12$ & $1 \mathrm{E}-12$ & $1 \mathrm{E}-12$ & $1 \mathrm{E}-12$ & $1 \mathrm{E}-12$ & $3,92 \mathrm{E}+30$ & $9,71 \mathrm{E}+30$ & $7,21 \mathrm{E}+33$ & $1 \mathrm{E}-12$ & 0,009 & 25,61739 & $1 \mathrm{E}-12$ & $1 \mathrm{E}-12$ & $1 \mathrm{E}-12$ & $1 \mathrm{E}-12$ \\
\hline 8 & $1 \mathrm{E}-12$ & $1 \mathrm{E}-12$ & $1 \mathrm{E}-12$ & $1 \mathrm{E}-12$ & $1 \mathrm{E}-12$ & $1 \mathrm{E}-12$ & 475749,6 & 1179397 & $8,76 \mathrm{E}+08$ & $1 \mathrm{E}-12$ & 0,009 & 25,61739 & $1 \mathrm{E}-12$ & $1 \mathrm{E}-12$ & $1 \mathrm{E}-12$ & $1 \mathrm{E}-12$ \\
\hline 9 & 44,75242 & 50,04043 & 101,3355 & 183,6749 & $1,88 \mathrm{E}+11$ & 0,009035 & 1,31E-19 & $3,25 \mathrm{E}-19$ & $2,21 \mathrm{E}-16$ & $1 \mathrm{E}-12$ & 0,009 & 25,61739 & $1 \mathrm{E}-12$ & $1 \mathrm{E}-12$ & $1 \mathrm{E}-12$ & $1 \mathrm{E}-12$ \\
\hline 10 & $1 \mathrm{E}-12$ & $1 \mathrm{E}-12$ & $1 \mathrm{E}-12$ & $1 \mathrm{E}-12$ & $1 \mathrm{E}-12$ & $1 \mathrm{E}-12$ & $5,28 \mathrm{E}+13$ & $5,28 \mathrm{E}+13$ & $1 \mathrm{E}-12$ & $1 \mathrm{E}-12$ & 0,009 & 25,61739 & $1 \mathrm{E}-12$ & $1 \mathrm{E}-12$ & $1 \mathrm{E}-12$ & $1 \mathrm{E}-12$ \\
\hline 11 & 44,75242 & 50,05475 & 101,3614 & 182,5373 & $7,4 \mathrm{E}+10$ & 0,009034 & $1 \mathrm{E}-12$ & $1 \mathrm{E}-12$ & $4,35 \mathrm{E}-24$ & $1 \mathrm{E}-12$ & 0,009 & 25,61739 & $1 \mathrm{E}-12$ & $9,99 \mathrm{E}-13$ & $9,99 \mathrm{E}-13$ & $3,22 \mathrm{E}+12$ \\
\hline 12 & $1 \mathrm{E}-12$ & $1 \mathrm{E}-12$ & $1 \mathrm{E}-12$ & $1 \mathrm{E}-12$ & 1E-12 & $1 \mathrm{E}-12$ & $4,81 \mathrm{E}+38$ & $7,57 \mathrm{E}+38$ & 1E-12 & $1 \mathrm{E}-12$ & 0,009 & 25,61739 & $1 \mathrm{E}-12$ & $9,99 \mathrm{E}-13$ & $9,99 \mathrm{E}-13$ & $1 \mathrm{E}-12$ \\
\hline 13 & $1 \mathrm{E}-12$ & $1 \mathrm{E}-12$ & $1 \mathrm{E}-12$ & $1 \mathrm{E}-12$ & $1 \mathrm{E}-12$ & $1 \mathrm{E}-12$ & $5,84 \mathrm{E}+13$ & $9,19 \mathrm{E}+13$ & $1,21 \mathrm{E}-37$ & $1 \mathrm{E}-12$ & 0,009 & 25,61739 & $1 \mathrm{E}-12$ & $9,99 \mathrm{E}-13$ & $9,99 \mathrm{E}-13$ & $1 \mathrm{E}-12$ \\
\hline 14 & 44,64243 & 49,94411 & 101,3298 & 181,6301 & $3 \mathrm{E}+10$ & 0,009034 & $1,52 \mathrm{E}-22$ & 1E-12 & $1,16 \mathrm{E}-24$ & $1 \mathrm{E}-12$ & 0,009 & 25,61739 & $1 \mathrm{E}-12$ & $9,99 \mathrm{E}-13$ & $9,99 \mathrm{E}-13$ & $8,36 \mathrm{E}+11$ \\
\hline 15 & $1 \mathrm{E}-12$ & $1 \mathrm{E}-12$ & $1 \mathrm{E}-12$ & $1 \mathrm{E}-12$ & $1 \mathrm{E}-12$ & $1 \mathrm{E}-12$ & $2,52 \mathrm{E}+38$ & $6,9 \mathrm{E}+38$ & $1 \mathrm{E}-12$ & $1 \mathrm{E}-12$ & 0,009 & 25,61739 & $1 \mathrm{E}-12$ & $9,99 \mathrm{E}-13$ & $9,99 \mathrm{E}-13$ & $1 \mathrm{E}-12$ \\
\hline 16 & $1 \mathrm{E}-12$ & $1 \mathrm{E}-12$ & $1 \mathrm{E}-12$ & $1 \mathrm{E}-12$ & $1 \mathrm{E}-12$ & $1 \mathrm{E}-12$ & $3,06 \mathrm{E}+13$ & $8,38 \mathrm{E}+13$ & $1,21 \mathrm{E}-37$ & $1 \mathrm{E}-12$ & 0,009 & 25,61739 & $1 \mathrm{E}-12$ & $9,99 \mathrm{E}-13$ & $9,99 \mathrm{E}-13$ & $1 \mathrm{E}-12$ \\
\hline 17 & 44,64243 & 49,95845 & 101,3558 & 180,4961 & $1,21 \mathrm{E}+10$ & 0,009032 & $1,42 \mathrm{E}-21$ & $1,2 \mathrm{E}-21$ & $1,16 \mathrm{E}-24$ & $1 \mathrm{E}-12$ & 0,009 & 25,61739 & $1 \mathrm{E}-12$ & $9,99 \mathrm{E}-13$ & $9,99 \mathrm{E}-13$ & $1 \mathrm{E}-12$ \\
\hline 18 & $1 \mathrm{E}-12$ & $1 \mathrm{E}-12$ & $1 \mathrm{E}-12$ & $1 \mathrm{E}-12$ & $1 \mathrm{E}-12$ & $1 \mathrm{E}-12$ & $1,02 \mathrm{E}+38$ & $5,32 \mathrm{E}+38$ & $1 \mathrm{E}-12$ & $1 \mathrm{E}-12$ & 0,009 & 25,61739 & $1 \mathrm{E}-12$ & $9,99 \mathrm{E}-13$ & $9,99 \mathrm{E}-13$ & $1 \mathrm{E}-12$ \\
\hline 19 & $1 \mathrm{E}-12$ & $1 \mathrm{E}-12$ & $1 \mathrm{E}-12$ & $1 \mathrm{E}-12$ & $1 \mathrm{E}-12$ & $1 \mathrm{E}-12$ & $1,24 \mathrm{E}+13$ & $6,46 \mathrm{E}+13$ & $1,21 \mathrm{E}-37$ & $1 \mathrm{E}-12$ & 0,009 & 25,61739 & $1 \mathrm{E}-12$ & $9,99 \mathrm{E}-13$ & $9,99 \mathrm{E}-13$ & $1 \mathrm{E}-12$ \\
\hline 20 & 44,64243 & 49,97281 & 101,3817 & 179,3643 & $4,85 \mathrm{E}+09$ & 0,00903 & $2,12 \mathrm{E}-21$ & $4,25 \mathrm{E}-21$ & $1,52 \mathrm{E}-24$ & $1 \mathrm{E}-12$ & 0,009 & 25,61739 & $1 \mathrm{E}-12$ & $9,99 \mathrm{E}-13$ & $9,99 \mathrm{E}-13$ & $1 \mathrm{E}-12$ \\
\hline 7.996 & 0,424933 & 0,480965 & 1,043938 & 1,455777 & $1,11 \mathrm{E}+16$ & $9,34 \mathrm{E}-05$ & $4,82 \mathrm{E}-08$ & 0,044673 & $6,37 \mathrm{E}+17$ & $1 \mathrm{E}-12$ & 0,009 & 25,61739 & $1 \mathrm{E}-12$ & $9,92 \mathrm{E}-13$ & $9,92 \mathrm{E}-13$ & 3,405581 \\
\hline 7.997 & $1 \mathrm{E}-12$ & $1 \mathrm{E}-12$ & $1 \mathrm{E}-12$ & $1 \mathrm{E}-12$ & $1 \mathrm{E}-12$ & $1 \mathrm{E}-12$ & $9,73 \mathrm{E}-50$ & $8,29 \mathrm{E}-47$ & $1 \mathrm{E}-12$ & $1 \mathrm{E}-12$ & 0,009 & 25,61739 & $1 \mathrm{E}-12$ & $9,92 \mathrm{E}-13$ & $9,92 \mathrm{E}-13$ & $1 \mathrm{E}-12$ \\
\hline 7.998 & 0,42804 & 0,484483 & 1,051595 & 1,466373 & $1,11 \mathrm{E}+16$ & $9,41 \mathrm{E}-05$ & $3,18 \mathrm{E}+20$ & $6,01 \mathrm{E}+23$ & $8,53 \mathrm{E}+42$ & $1 \mathrm{E}-12$ & 0,009 & 25,61739 & $1 \mathrm{E}-12$ & $9,92 \mathrm{E}-13$ & $9,92 \mathrm{E}-13$ & 3,430449 \\
\hline 7.999 & 0,42804 & 0,484483 & 1,051595 & 1,466373 & $1,11 \mathrm{E}+16$ & $9,41 \mathrm{E}-05$ & $3,86 \mathrm{E}-05$ & 0,073008 & $1,04 \mathrm{E}+18$ & $1 \mathrm{E}-12$ & 0,009 & 25,61739 & $1 \mathrm{E}-12$ & $9,92 \mathrm{E}-13$ & $9,92 \mathrm{E}-13$ & 3,430449 \\
\hline 8.000 & $1 \mathrm{E}-12$ & $1 \mathrm{E}-12$ & $1 \mathrm{E}-12$ & $1 \mathrm{E}-12$ & $1 \mathrm{E}-12$ & $1 \mathrm{E}-12$ & $1 \mathrm{E}-12$ & $1 \mathrm{E}-47$ & $1 \mathrm{E}-12$ & $1 \mathrm{E}-12$ & 0,009 & 25,61739 & $1 \mathrm{E}-12$ & $9,92 \mathrm{E}-13$ & $9,92 \mathrm{E}-13$ & $1 \mathrm{E}-12$ \\
\hline
\end{tabular}

Fonte: Própria autoria. 
Tabela 24 - Simulação feita com 80.000 iterações (continua)

\begin{tabular}{|c|c|c|c|c|c|c|c|c|c|c|c|c|c|c|c|c|}
\hline Iteração & $S_{\mathrm{su}}$ & $S_{\text {va }}$ & ${ }^{S} \mathrm{bu}$ & $S_{\text {pro }}$ & $S_{\mathrm{ac}}$ & ${ }^{S_{\mathrm{h} 2}}$ & $S_{\operatorname{ch} 4}$ & ${ }^{{ }^{S} \mathrm{IC}}$ & ${ }^{S}$ IN & $X_{\mathrm{Su}}$ & $x_{\mathrm{c} 4}$ & $X_{\mathrm{pr}}$ & $X_{\mathrm{ac}}$ & $x_{\mathrm{h} 2}$ & ${ }^{S_{\text {cat }}}$ & $S_{\text {an }}$ \\
\hline 0 & 1,15 & 0,052 & 0,053 & 0,123 & 0,171 & $1 \mathrm{E}-09$ & 0,000001 & 52678 & 3 & 0,012016 & 0,01 & 0,003 & 0,04126 & 0,31702 & 0,04 & 0,02 \\
\hline 1 & 1,15 & 0,052 & 0,053 & 0,123 & 0,171 & 4,36E-09 & $9,89 \mathrm{E}-07$ & 0,152522 & 0,13023 & 0,012016 & 0,01 & 0,003 & 0,04126 & 0,317019 & 0,04 & 0,02 \\
\hline 2 & 1,15 & 0,052 & 0,053 & 0,123 & 0,171 & 4,36E-09 & $9,89 \mathrm{E}-07$ & 0,152522 & 0,13023 & 0,012016 & 0,01 & 0,003 & 0,04126 & 0,317019 & 0,04 & 0,02 \\
\hline 3 & 1,149987 & 0,051987 & 0,052988 & 0,122996 & 0,170916 & 4983,363 & 7841,103 & 251776,5 & 0,13023 & 0,012017 & 0,010001 & 0,003 & 0,041265 & 0,317016 & 0,04 & 0,02 \\
\hline 4 & 1,149987 & 0,051987 & 0,052988 & 0,122996 & 0,170916 & 4912,786 & 7730,053 & 248210,7 & 0,13023 & 0,012017 & 0,010001 & 0,003 & 0,041265 & 0,317015 & 0,04 & 0,02 \\
\hline 5 & 1,149987 & 0,051987 & 0,052988 & 0,122996 & 0,170916 & 4912,786 & 7730,053 & 248210,7 & 0,13023 & 0,012017 & 0,010001 & 0,003 & 0,041265 & 0,317015 & 0,04 & 0,02 \\
\hline 6 & 1,149974 & 0,051987 & 0,05299 & 0,122999 & 0,170811 & 128409,7 & 314023,1 & $2,31 \mathrm{E}+08$ & 0,130228 & 0,012018 & 0,010001 & 0,003 & 0,04127 & 0,317427 & 0,04 & 0,02 \\
\hline 7 & 1,149974 & 0,051987 & 0,05299 & 0,122999 & 0,170811 & 126682,3 & 309798,8 & $2,28 \mathrm{E}+08$ & 0,130228 & 0,012018 & 0,010001 & 0,003 & 0,04127 & 0,317427 & 0,04 & 0,02 \\
\hline 8 & 1,149974 & 0,051987 & 0,05299 & 0,122999 & 0,170811 & 126682,3 & 309798,8 & $2,28 \mathrm{E}+08$ & 0,130228 & 0,012018 & 0,010001 & 0,003 & 0,04127 & 0,317427 & 0,04 & 0,02 \\
\hline 9 & 1,149961 & 0,051987 & 0,052991 & 0,123002 & 0,170706 & 187029,6 & 564203,2 & $5,21 \mathrm{E}+09$ & 0,130225 & 0,012019 & 0,010001 & 0,003 & 0,041275 & 0,317839 & 0,04 & 0,02 \\
\hline 10 & 1,149961 & 0,051987 & 0,052991 & 0,123002 & 0,170706 & 184561,7 & 556758,2 & $5,15 \mathrm{E}+09$ & 0,130225 & 0,012019 & 0,010001 & 0,003 & 0,041275 & 0,317839 & 0,04 & 0,02 \\
\hline 11 & 1,149961 & 0,051987 & 0,052991 & 0,123002 & 0,170706 & 184561,7 & 556758,2 & $5,15 \mathrm{E}+09$ & 0,130225 & 0,012019 & 0,010001 & 0,003 & 0,041275 & 0,317839 & 0,04 & 0,02 \\
\hline 12 & 1,149948 & 0,051987 & 0,052993 & 0,123006 & 0,170601 & 190245,6 & 623642,6 & $3 E+10$ & 0,130223 & 0,01202 & 0,010001 & 0,003 & 0,04128 & 0,318252 & 0,04 & 0,02 \\
\hline 13 & 1,149948 & 0,051987 & 0,052993 & 0,123006 & 0,170601 & 187761,1 & 615498 & $2,98 \mathrm{E}+10$ & 0,130223 & 0,01202 & 0,010001 & 0,003 & 0,04128 & 0,318251 & 0,04 & 0,02 \\
\hline 14 & 1,149948 & 0,051987 & 0,052993 & 0,123006 & 0,170601 & 187761,1 & 615498 & $2,98 \mathrm{E}+10$ & 0,130223 & 0,01202 & 0,010001 & 0,003 & 0,04128 & 0,318251 & 0,04 & 0,02 \\
\hline 15 & 1,149935 & 0,051987 & 0,052994 & 0,123009 & 0,170496 & 178609 & 612410 & $6,51 \mathrm{E}+10$ & 0,13022 & 0,012022 & 0,010001 & 0,003 & 0,041285 & 0,318664 & 0,04 & 0,02 \\
\hline 16 & 1,149935 & 0,051987 & 0,052994 & 0,123009 & 0,170496 & 176308,2 & 604521,1 & $6,55 \mathrm{E}+10$ & 0,13022 & 0,012022 & 0,010001 & 0,003 & 0,041285 & 0,318664 & 0,04 & 0,02 \\
\hline 17 & 1,149935 & 0,051987 & 0,052994 & 0,123009 & 0,170496 & 176308,2 & 604521,1 & $6,55 \mathrm{E}+10$ & 0,13022 & 0,012022 & 0,010001 & 0,003 & 0,041285 & 0,318664 & 0,04 & 0,02 \\
\hline 18 & 1,149923 & 0,051987 & 0,052996 & 0,123012 & 0,170391 & 159541,1 & 557394,6 & $5,73 \mathrm{E}+10$ & 0,130218 & 0,012023 & 0,010001 & 0,003 & 0,041289 & 0,319078 & 0,04 & 0,02 \\
\hline 19 & 1,149923 & 0,051987 & 0,052996 & 0,123012 & 0,170391 & 157493,1 & 550239,2 & $5,91 \mathrm{E}+10$ & 0,130218 & 0,012023 & 0,010001 & 0,003 & 0,041289 & 0,319078 & 0,04 & 0,02 \\
\hline 20 & 1,149923 & 0,051987 & 0,052996 & 0,123012 & 0,170391 & 157493,1 & 550239,2 & $5,91 \mathrm{E}+10$ & 0,130218 & 0,012023 & 0,010001 & 0,003 & 0,041289 & 0,319078 & 0,04 & 0,02 \\
\hline 79.996 & 0,947132 & 0,006055 & 0,011035 & 0,082333 & 0,016611 & $1,13 \mathrm{E}-06$ & $1,09 \mathrm{E}+15$ & $4,9 \mathrm{E}+09$ & $8,81 \mathrm{E}-06$ & 0,026891 & 0,013509 & 0,006267 & 0,047195 & 21,62843 & 0,04 & 0,02 \\
\hline 79.997 & 0,947132 & 0,006055 & 0,011035 & 0,082333 & 0,016611 & $1,13 \mathrm{E}-06$ & $1,09 \mathrm{E}+15$ & $4,9 \mathrm{E}+09$ & $8,81 \mathrm{E}-06$ & 0,026891 & 0,013509 & 0,006267 & 0,047195 & 21,62843 & 0,04 & 0,02 \\
\hline 79.998 & 0,94713 & 0,006055 & 0,011035 & 0,082333 & 0,016611 & $1 \mathrm{E}-12$ & $1,06 \mathrm{E}+15$ & $4,29 \mathrm{E}+09$ & 7,8E-06 & 0,026891 & 0,013508 & 0,006267 & 0,047194 & 21,62848 & 0,04 & 0,02 \\
\hline 79.999 & 0,94713 & 0,006055 & 0,011035 & 0,082333 & 0,016611 & $9,87 \mathrm{E}-13$ & $1,04 \mathrm{E}+15$ & $4,43 \mathrm{E}+09$ & $7,89 \mathrm{E}-06$ & 0,026891 & 0,013508 & 0,006267 & 0,047194 & 21,62846 & 0,04 & 0,02 \\
\hline 80.000 & 0,94713 & 0,006055 & 0,011035 & 0,082333 & 0,016611 & $9,87 \mathrm{E}-13$ & $1,04 \mathrm{E}+15$ & $4,43 \mathrm{E}+09$ & $7,89 \mathrm{E}-06$ & 0,026891 & 0,013508 & 0,006267 & 0,047194 & 21,62846 & 0,04 & 0,02 \\
\hline
\end{tabular}


Tabela 24 - Simulação feita com 80.000 iterações (conclusão)

\begin{tabular}{|c|c|c|c|c|c|c|c|c|c|c|c|c|c|c|c|c|}
\hline Iteração & $s_{\text {vam }}$ & ${ }^{s}$ bum & $S_{\text {prom }}$ & $s_{\mathrm{acm}}$ & ${ }^{S}$ hco3m & ${ }^{S}$ nh3 & $s_{\text {gas, h2 }}$ & $S_{\text {gas,ch4 }}$ & $s_{\text {gas,co } 2}$ & $s_{\mathrm{ca}}$ & ${ }^{S_{\mathrm{I}}}$ & ${ }^{X} \mathbf{I}$ & ${ }^{S}$ lac & ${ }^{X_{\text {lac }, \mathrm{f}}}$ & ${ }^{X}$ lac,o & ${ }^{S} \mathrm{DQO}$ \\
\hline 0 & 0,052 & 0,053 & 0,123 & 0,171 & 0,142777 & 0,00409 & 0,00001 & 0,00001 & 0,00001 & 0,00001 & 0,009 & 25,61739 & 0 & 0 & 0 & 2,7 \\
\hline 1 & $1 \mathrm{E}-12$ & $1 \mathrm{E}-12$ & $1 \mathrm{E}-12$ & $1 \mathrm{E}-12$ & $1 \mathrm{E}-12$ & $1 \mathrm{E}-12$ & $1,73 \mathrm{E}+31$ & $1,73 \mathrm{E}+31$ & $2,38 \mathrm{E}+31$ & $1 \mathrm{E}-05$ & 0,009 & 25,61739 & $1 \mathrm{E}-12$ & $1 \mathrm{E}-12$ & $1 \mathrm{E}-12$ & $1 \mathrm{E}-12$ \\
\hline 2 & $1 \mathrm{E}-12$ & $1 \mathrm{E}-12$ & $1 \mathrm{E}-12$ & $1 \mathrm{E}-12$ & $1 \mathrm{E}-12$ & $1 \mathrm{E}-12$ & 2107096 & 2107167 & 2895941 & $1 \mathrm{E}-05$ & 0,009 & 25,61739 & $1 \mathrm{E}-12$ & $1 \mathrm{E}-12$ & $1 \mathrm{E}-12$ & $1 \mathrm{E}-12$ \\
\hline 3 & 4,486249 & 5,013672 & 10,1341 & 18,57275 & 0,470631 & 0,000904 & $1 \mathrm{E}-12$ & $1 \mathrm{E}-12$ & $1 \mathrm{E}-12$ & $1 \mathrm{E}-05$ & 0,009 & 25,61739 & $1 \mathrm{E}-12$ & $1 \mathrm{E}-12$ & $1 \mathrm{E}-12$ & 12862,67 \\
\hline 4 & $1 \mathrm{E}-12$ & $1 \mathrm{E}-12$ & $1 \mathrm{E}-12$ & $1 \mathrm{E}-12$ & $1 \mathrm{E}-12$ & $1 \mathrm{E}-12$ & $4,32 \mathrm{E}+32$ & $6,8 \mathrm{E}+32$ & $2,18 \mathrm{E}+34$ & $1 \mathrm{E}-05$ & 0,009 & 25,61739 & $1 \mathrm{E}-12$ & $1 \mathrm{E}-12$ & $1 \mathrm{E}-12$ & $1 \mathrm{E}-12$ \\
\hline 5 & $1 \mathrm{E}-12$ & $1 \mathrm{E}-12$ & $1 \mathrm{E}-12$ & $1 \mathrm{E}-12$ & $1 \mathrm{E}-12$ & $1 \mathrm{E}-12$ & 52477388 & 82570859 & $2,65 E+09$ & $1 \mathrm{E}-05$ & 0,009 & 25,61739 & $1 \mathrm{E}-12$ & $1 \mathrm{E}-12$ & $1 \mathrm{E}-12$ & $1 \mathrm{E}-12$ \\
\hline 6 & 4,485148 & 5,012566 & 10,13379 & 18,56362 & 765896 & 0,000904 & $1 \mathrm{E}-12$ & $1 \mathrm{E}-12$ & $1 \mathrm{E}-12$ & $1 \mathrm{E}-05$ & 0,009 & 25,61739 & $1 E-12$ & $1 \mathrm{E}-12$ & $1 \mathrm{E}-12$ & 429828,2 \\
\hline 7 & $1 \mathrm{E}-12$ & $1 \mathrm{E}-12$ & $1 \mathrm{E}-12$ & $1 \mathrm{E}-12$ & $1 \mathrm{E}-12$ & $1 \mathrm{E}-12$ & $2,65 E+32$ & $6,48 E+32$ & $4,75 E+35$ & $1 \mathrm{E}-12$ & 0,009 & 25,61739 & $1 \mathrm{E}-12$ & $1 \mathrm{E}-12$ & $1 \mathrm{E}-12$ & $1 \mathrm{E}-12$ \\
\hline 8 & $1 \mathrm{E}-12$ & $1 \mathrm{E}-12$ & $1 \mathrm{E}-12$ & $1 \mathrm{E}-12$ & $1 \mathrm{E}-12$ & $1 \mathrm{E}-12$ & 32211970 & 78773640 & $5,77 \mathrm{E}+10$ & $1 \mathrm{E}-12$ & 0,009 & 25,61739 & $1 \mathrm{E}-12$ & $1 \mathrm{E}-12$ & $1 \mathrm{E}-12$ & $1 \mathrm{E}-12$ \\
\hline 9 & 4,485148 & 5,012709 & 10,13404 & 18,55221 & $7,02 \mathrm{E}+08$ & 0,000904 & $1 \mathrm{E}-12$ & $1 \mathrm{E}-12$ & $1 \mathrm{E}-12$ & $1 \mathrm{E}-12$ & 0,009 & 25,61739 & $1 \mathrm{E}-12$ & $1 \mathrm{E}-12$ & $1 \mathrm{E}-12$ & 314789,8 \\
\hline 10 & $1 \mathrm{E}-12$ & $1 \mathrm{E}-12$ & $1 \mathrm{E}-12$ & $1 \mathrm{E}-12$ & $1 \mathrm{E}-12$ & $1 \mathrm{E}-12$ & $1 \mathrm{E}+32$ & $3,02 \mathrm{E}+32$ & $2,41 \mathrm{E}+36$ & $1 \mathrm{E}-12$ & 0,009 & 25,61739 & $1 \mathrm{E}-12$ & $1 \mathrm{E}-12$ & $1 \mathrm{E}-12$ & $1 \mathrm{E}-12$ \\
\hline 11 & $1 \mathrm{E}-12$ & $1 \mathrm{E}-12$ & $1 \mathrm{E}-12$ & $1 \mathrm{E}-12$ & $1 \mathrm{E}-12$ & $1 \mathrm{E}-12$ & 12158019 & 36676511 & $2,93 \mathrm{E}+11$ & $1 \mathrm{E}-12$ & 0,009 & 1739 & $1 \mathrm{E}-12$ & $1 \mathrm{E}-12$ & $1 \mathrm{E}-12$ & $1 \mathrm{E}-12$ \\
\hline 12 & 4,485148 & 5,012852 & 10,1343 & 18,5408 & $1,59 \mathrm{E}+10$ & 0,000904 & $1 \mathrm{E}-12$ & $1 \mathrm{E}-12$ & $1 \mathrm{E}-12$ & $1 \mathrm{E}-12$ & 0,009 & 25,61739 & $1 \mathrm{E}-12$ & $1 \mathrm{E}-12$ & $1 \mathrm{E}-12$ & 72606,49 \\
\hline 13 & $1 \mathrm{E}-12$ & $1 \mathrm{E}-12$ & $1 \mathrm{E}-12$ & $1 \mathrm{E}-12$ & $1 \mathrm{E}-12$ & $1 \mathrm{E}-12$ & $4,98 \mathrm{E}+31$ & $1,63 \mathrm{E}+32$ & $3,7 \mathrm{E}+36$ & $1 \mathrm{E}-12$ & 0,009 & 25,61739 & $1 \mathrm{E}-12$ & $1 \mathrm{E}-12$ & $1 \mathrm{E}-12$ & $1 \mathrm{E}-12$ \\
\hline 14 & $1 \mathrm{E}-12$ & $1 \mathrm{E}-12$ & $1 \mathrm{E}-12$ & $1 \mathrm{E}-12$ & $1 \mathrm{E}-12$ & $1 \mathrm{E}-12$ & 6054059 & 19845759 & $4,49 \mathrm{E}+11$ & $1 \mathrm{E}-12$ & 0,009 & 25,61739 & $1 \mathrm{E}-12$ & $1 \mathrm{E}-12$ & $1 \mathrm{E}-12$ & $1 \mathrm{E}-12$ \\
\hline 15 & 4,485148 & 5,012995 & 10,13456 & 18,5294 & $9,2 \mathrm{E}+10$ & 0,000904 & $1,12 \mathrm{E}-26$ & $3,78 \mathrm{E}-27$ & $1 \mathrm{E}-12$ & $1 \mathrm{E}-12$ & 0,009 & 25,61739 & $1 \mathrm{E}-12$ & $1 \mathrm{E}-12$ & $1 \mathrm{E}-12$ & $1 \mathrm{E}-12$ \\
\hline 16 & $1 \mathrm{E}-12$ & $1 \mathrm{E}-12$ & $1 \mathrm{E}-12$ & $1 \mathrm{E}-12$ & $1 \mathrm{E}-12$ & $1 \mathrm{E}-12$ & $1,83 \mathrm{E}+31$ & $6,29 \mathrm{E}+31$ & $1 \mathrm{E}-12$ & $1 \mathrm{E}-12$ & 0,009 & 25,61739 & $1 \mathrm{E}-12$ & $1 \mathrm{E}-12$ & $1 \mathrm{E}-12$ & $1 \mathrm{E}-12$ \\
\hline 17 & $1 \mathrm{E}-12$ & $1 \mathrm{E}-12$ & $1 \mathrm{E}-12$ & $1 \mathrm{E}-12$ & $1 \mathrm{E}-12$ & $1 \mathrm{E}-12$ & 2228904 & 7642407 & $1,21 \mathrm{E}-37$ & $1 \mathrm{E}-12$ & 0,009 & 25,61739 & $1 \mathrm{E}-12$ & $1 \mathrm{E}-12$ & $1 \mathrm{E}-12$ & $1 \mathrm{E}-12$ \\
\hline 18 & 4,485148 & 5,013137 & 10,13482 & 18,518 & $2,02 \mathrm{E}+11$ & 0,000904 & $6,18 \mathrm{E}-14$ & $1,74 \mathrm{E}-13$ & $3,02 \mathrm{E}-08$ & $1 \mathrm{E}-12$ & 0,009 & 25,61739 & $1 \mathrm{E}-12$ & $1 \mathrm{E}-12$ & $1 \mathrm{E}-12$ & $1 \mathrm{E}-12$ \\
\hline 19 & $1 \mathrm{E}-12$ & $1 \mathrm{E}-12$ & $1 \mathrm{E}-12$ & $1 \mathrm{E}-12$ & $1 \mathrm{E}-12$ & $1 \mathrm{E}-12$ & $1,3 \mathrm{E}+31$ & $4,53 \mathrm{E}+31$ & $1 \mathrm{E}-12$ & $1 \mathrm{E}-12$ & 0,009 & 25,61739 & $1 \mathrm{E}-12$ & $1 \mathrm{E}-12$ & $1 \mathrm{E}-12$ & $1 \mathrm{E}-12$ \\
\hline 20 & $1 \mathrm{E}-12$ & $1 \mathrm{E}-12$ & $1 \mathrm{E}-12$ & $1 \mathrm{E}-12$ & $1 \mathrm{E}-12$ & $1 \mathrm{E}-12$ & 1576478 & 5507797 & $1,21 \mathrm{E}-37$ & $1 \mathrm{E}-12$ & 0,009 & 25,61739 & $1 \mathrm{E}-12$ & $1 \mathrm{E}-12$ & $1 \mathrm{E}-12$ & $1 \mathrm{E}-12$ \\
\hline 79.996 & $1 \mathrm{E}-12$ & $1 \mathrm{E}-12$ & $1 \mathrm{E}-12$ & $1 \mathrm{E}-12$ & $1 \mathrm{E}-12$ & $1 \mathrm{E}-12$ & $2,42 \mathrm{E}+20$ & $2,33 \mathrm{E}+41$ & $1 \mathrm{E}-12$ & $1 \mathrm{E}-12$ & 0,009 & 25,61739 & $1 \mathrm{E}-12$ & $7,88 \mathrm{E}-13$ & $7,88 \mathrm{E}-13$ & $1 \mathrm{E}-12$ \\
\hline 79.997 & $1 \mathrm{E}-12$ & $1 \mathrm{E}-12$ & $1 \mathrm{E}-12$ & $1 \mathrm{E}-12$ & $1 \mathrm{E}-12$ & $1 \mathrm{E}-12$ & 2,94E-05 & $2,83 \mathrm{E}+16$ & $1,21 \mathrm{E}-37$ & $1 \mathrm{E}-12$ & 0,009 & 25,61739 & $1 \mathrm{E}-12$ & $7,88 \mathrm{E}-13$ & $7,88 \mathrm{E}-13$ & $1 \mathrm{E}-12$ \\
\hline 79.998 & 0,522393 & 1,043908 & 6,783531 & 1,804116 & $1,51 \mathrm{E}+10$ & $6,11 \mathrm{E}-08$ & $9,04 \mathrm{E}-44$ & $3,86 \mathrm{E}-23$ & $7,71 \mathrm{E}-28$ & $1 \mathrm{E}-12$ & 0,009 & 25,61739 & $1 \mathrm{E}-12$ & $7,88 \mathrm{E}-13$ & $7,88 \mathrm{E}-13$ & $1 \mathrm{E}-12$ \\
\hline 79.999 & $1 \mathrm{E}-12$ & $1 \mathrm{E}-12$ & $1 \mathrm{E}-12$ & $1 \mathrm{E}-12$ & $1 \mathrm{E}-12$ & $1 \mathrm{E}-12$ & $2,96 \mathrm{E}+14$ & $3,13 \mathrm{E}+41$ & $1 \mathrm{E}-12$ & $1 \mathrm{E}-12$ & 0,009 & 25,61739 & $1 \mathrm{E}-12$ & $7,88 \mathrm{E}-13$ & $7,88 \mathrm{E}-13$ & $1 \mathrm{E}-12$ \\
\hline 80.000 & $1 \mathrm{E}-12$ & $1 \mathrm{E}-12$ & $1 \mathrm{E}-12$ & $1 \mathrm{E}-12$ & $1 \mathrm{E}-12$ & $1 \mathrm{E}-12$ & $3,59 \mathrm{E}-11$ & $3,8 \mathrm{E}+16$ & $1,21 \mathrm{E}-37$ & $1 \mathrm{E}-12$ & 0,009 & 25,61739 & $1 \mathrm{E}-12$ & $7,88 \mathrm{E}-13$ & $7,88 \mathrm{E}-13$ & $1 \mathrm{E}-12$ \\
\hline
\end{tabular}

Fonte: Própria autoria. 
Tabela 25 - Simulação feita com 800.000 iterações (continua)

\begin{tabular}{|c|c|c|c|c|c|c|c|c|c|c|c|c|c|c|c|c|}
\hline Iteração & $S_{\mathrm{Su}}$ & $S_{\mathrm{va}}$ & ${ }^{S} \mathrm{bu}$ & $S_{\text {pro }}$ & $S_{\mathrm{ac}}$ & ${ }^{S_{\mathrm{h} 2}}$ & $S^{S} \operatorname{ch} 4$ & ${ }^{S_{\mathrm{IC}}}$ & ${ }^{S}$ IN & $X_{\mathrm{su}}$ & $x_{\mathrm{c} 4}$ & ${ }^{X} \mathrm{pr}$ & $X_{\mathrm{ac}}$ & ${ }^{x_{\mathrm{h} 2}}$ & $S_{\text {cat }}$ & $S_{\text {an }}$ \\
\hline 0 & 1,15 & 0,052 & 0,053 & 0,123 & 0,171 & $1 \mathrm{E}-09$ & 0,000001 & 0,152678 & 0,13023 & 0,012016 & 0,01 & 0,003 & 0,04126 & 0,31702 & 0,04 & 0,02 \\
\hline 1 & 1,15 & 0,052 & 0,053 & 0,123 & 0,171 & $3,66 \mathrm{E}-09$ & 9,91E-07 & 0,152554 & 0,13023 & 0,012016 & 0,01 & 0,003 & 0,04126 & 0,31702 & 0,04 & 0,02 \\
\hline 2 & 1,15 & 0,052 & 0,053 & 0,123 & 0,171 & $3,66 \mathrm{E}-09$ & 9,91E-07 & 0,152554 & 0,13023 & 0,012016 & 0,01 & 0,003 & 0,04126 & 0,31702 & 0,04 & 0,02 \\
\hline 3 & 1,15 & 0,052 & 0,053 & 0,123 & 0,170997 & $1,72 \mathrm{E}-06$ & $6,88 \mathrm{E}-06$ & 0,151983 & 0,13023 & 0,012016 & 0,01 & 0,003 & 0,04126 & 0,317019 & 0,04 & 0,02 \\
\hline 4 & 1,15 & 0,052 & 0,053 & 0,123 & 0,170997 & $1,72 \mathrm{E}-06$ & $6,88 \mathrm{E}-06$ & 0,151983 & 0,13023 & 0,012016 & 0,01 & 0,003 & 0,04126 & 0,317019 & 0,04 & 0,02 \\
\hline 5 & 1,149998 & 0,051998 & 0,052999 & 0,123 & 0,170988 & 39852611 & 62706262 & $2,01 \mathrm{E}+09$ & 0,13023 & 0,012016 & 0,01 & 0,003 & 0,041261 & 0,317027 & 0,04 & 0,02 \\
\hline 6 & 1,149997 & 0,051998 & 0,052999 & 0,123 & 0,170979 & 39354453 & 61922434 & $1,99 \mathrm{E}+09$ & 0,13023 & 0,012016 & 0,01 & 0,003 & 0,041261 & 0,317068 & 0,04 & 0,02 \\
\hline 7 & 1,149997 & 0,051998 & 0,052999 & 0,123 & 0,170979 & 39354453 & 61922434 & $1,99 \mathrm{E}+09$ & 0,13023 & 0,012016 & 0,01 & 0,003 & 0,041261 & 0,317068 & 0,04 & 0,02 \\
\hline 8 & 1,149997 & 0,051998 & 0,052999 & 0,123 & 0,170979 & 38878309 & 61187486 & $2 \mathrm{E}+09$ & 0,13023 & 0,012016 & 0,01 & 0,003 & 0,041261 & 0,317068 & 0,04 & 0,02 \\
\hline 9 & 1,149996 & 0,051998 & 0,052999 & 0,123 & 0,170968 & 38392330 & 60422642 & $1,98 \mathrm{E}+09$ & 0,130229 & 0,012016 & 0,01 & 0,003 & 0,041262 & 0,317109 & 0,04 & 0,02 \\
\hline 10 & 1,149996 & 0,051998 & 0,052999 & 0,123 & 0,170968 & 38392330 & 60422642 & $1,98 \mathrm{E}+09$ & 0,130229 & 0,012016 & 0,01 & 0,003 & 0,041262 & 0,317109 & 0,04 & 0,02 \\
\hline 11 & 1,149996 & 0,051998 & 0,052999 & 0,123 & 0,170968 & 37927827 & 59705495 & $1,99 \mathrm{E}+09$ & 0,130229 & 0,012016 & 0,01 & 0,003 & 0,041261 & 0,317109 & 0,04 & 0,02 \\
\hline 12 & 1,149994 & 0,051998 & 0,052999 & 0,123001 & 0,170958 & 37453729 & 58959176 & $1,96 \mathrm{E}+09$ & 0,130229 & 0,012016 & 0,01 & 0,003 & 0,041262 & 0,31715 & 0,04 & 0,02 \\
\hline 13 & 1,149994 & 0,051998 & 0,052999 & 0,123001 & 0,170958 & 37453729 & 58959176 & $1,96 \mathrm{E}+09$ & 0,130229 & 0,012016 & 0,01 & 0,003 & 0,041262 & 0,31715 & 0,04 & 0,02 \\
\hline 14 & 1,149994 & 0,051998 & 0,052999 & 0,123001 & 0,170958 & 37000581 & 58259399 & $1,98 \mathrm{E}+09$ & 0,130229 & 0,012016 & 0,01 & 0,003 & 0,041262 & 0,31715 & 0,04 & 0,02 \\
\hline 15 & 1,149993 & 0,051998 & 0,052999 & 0,123001 & 0,170947 & 36538074 & 57531156 & $1,95 \mathrm{E}+09$ & 0,130229 & 0,012017 & 0,01 & 0,003 & 0,041262 & 0,317191 & 0,04 & 0,02 \\
\hline 16 & 1,149993 & 0,051998 & 0,052999 & 0,123001 & 0,170947 & 36538074 & 57531156 & $1,95 \mathrm{E}+09$ & 0,130229 & 0,012017 & 0,01 & 0,003 & 0,041262 & 0,317191 & 0,04 & 0,02 \\
\hline 17 & 1,149993 & 0,051998 & 0,052999 & 0,123001 & 0,170947 & 36096005 & 56848327 & $1,96 \mathrm{E}+09$ & 0,130229 & 0,012017 & 0,01 & 0,003 & 0,041262 & 0,31719 & 0,04 & 0,02 \\
\hline 18 & 1,149992 & 0,051998 & 0,052999 & 0,123001 & 0,170937 & 35644805 & 56137723 & $1,94 \mathrm{E}+09$ & 0,130229 & 0,012017 & 0,01 & 0,003 & 0,041263 & 0,317232 & 0,04 & 0,02 \\
\hline 19 & 1,149992 & 0,051998 & 0,052999 & 0,123001 & 0,170937 & 35644805 & 56137723 & $1,94 \mathrm{E}+09$ & 0,130229 & 0,012017 & 0,01 & 0,003 & 0,041263 & 0,317232 & 0,04 & 0,02 \\
\hline 20 & 1,149992 & 0,051998 & 0,052999 & 0,123001 & 0,170937 & 35213543 & 55471433 & $1,95 \mathrm{E}+09$ & 0,130229 & 0,012017 & 0,01 & 0,003 & 0,041263 & 0,317231 & 0,04 & 0,02 \\
\hline 799.996 & 0,002532 & 0,000576 & 0,004299 & 0,00812 & 0,008959 & $1 \mathrm{E}-12$ & 0,016121 & 0,188207 & 0,000176 & 0,096154 & 0,01492 & 0,013917 & 0,056252 & 20,91271 & 0,04 & 0,02 \\
\hline 799.997 & 0,002532 & 0,000576 & 0,004299 & 0,00812 & 0,008959 & $8,07 \mathrm{E}-11$ & 0,015926 & 0,188411 & 0,000176 & 0,096154 & 0,01492 & 0,013917 & 0,056252 & 20,91268 & 0,04 & 0,02 \\
\hline 799.998 & 0,002532 & 0,000576 & 0,004299 & 0,008119 & 0,008958 & $1 \mathrm{E}-12$ & 0,015728 & 0,186056 & 0,000177 & 0,096154 & 0,01492 & 0,013917 & 0,056252 & 20,91265 & 0,04 & 0,02 \\
\hline 799.999 & 0,002532 & 0,000576 & 0,004299 & 0,008119 & 0,008958 & $1 \mathrm{E}-12$ & 0,015728 & 0,186056 & 0,000177 & 0,096154 & 0,01492 & 0,013917 & 0,056252 & 20,91265 & 0,04 & 0,02 \\
\hline 800.000 & 0,002532 & 0,000576 & 0,004299 & 0,008119 & 0,008958 & $1,69 \mathrm{E}-12$ & 0,015538 & 0,186255 & 0,000177 & 0,096154 & 0,01492 & 0,013917 & 0,056251 & 20,91263 & 0,04 & 0,02 \\
\hline
\end{tabular}


Tabela 25 - Simulação feita com 800.000 iterações (conclusão)

\begin{tabular}{|c|c|c|c|c|c|c|c|c|c|c|c|c|c|c|c|c|}
\hline Iteração & $S_{\text {vam }}$ & $S_{\text {bum }}$ & $S_{\text {prom }}$ & $s_{\mathrm{acm}}$ & ${ }^{S}$ hco3m & $s_{\mathrm{nh} 3}$ & $s_{\text {gas, h2 }}$ & $S_{\text {gas,ch4 }}$ & ${ }^{s_{\text {gas, }, \mathrm{co} 2}}$ & $s_{\mathrm{ca}}$ & ${ }^{S_{\mathrm{I}}}$ & ${ }^{x_{\mathrm{I}}}$ & $S_{\text {lac }}$ & ${ }^{X}$ lac,f & ${ }^{x_{1} \text { lac,o }}$ & ${ }^{S} \mathrm{DQO}$ \\
\hline 0 & 0,052 & 0,053 & 0,123 & 0,171 & 0,142777 & 0,00409 & 0,00001 & 0,00001 & 0,00001 & 0,00001 & 0,009 & 25,61739 & 0 & 0 & 0 & 2,7 \\
\hline 1 & $1 \mathrm{E}-12$ & $1 \mathrm{E}-12$ & $1 \mathrm{E}-12$ & $1 \mathrm{E}-12$ & $1 \mathrm{E}-12$ & $1 \mathrm{E}-12$ & $4,94 \mathrm{E}+23$ & $4,94 \mathrm{E}+23$ & $6,79 \mathrm{E}+23$ & $1 \mathrm{E}-05$ & 0,009 & 25,61739 & E-12 & $1 \mathrm{E}-12$ & $1 \mathrm{E}-12$ & $1 \mathrm{E}-12$ \\
\hline 2 & $1 \mathrm{E}-12$ & $1 \mathrm{E}-12$ & $1 \mathrm{E}-12$ & $1 \mathrm{E}-12$ & $1 \mathrm{E}-12$ & $1 \mathrm{E}-12$ & 0,017956 & 0,017957 & 0,024679 & $1 \mathrm{E}-05$ & 0,009 & 25,61739 & E-12 & $1 \mathrm{E}-12$ & $1 \mathrm{E}-12$ & $1 \mathrm{E}-12$ \\
\hline 3 & 0,151558 &, 169376 & 0,342358 & 0,627438 & 0,015903 & $3,05 \mathrm{E}-05$ & $1,39 \mathrm{E}+36$ & $1,39 \mathrm{E}+36$ & $1,91 \mathrm{E}+36$ & $1 \mathrm{E}-05$ & 0,009 & 25,61739 & $1 \mathrm{E}-12$ & $1 \mathrm{E}-12$ & $1 \mathrm{E}-12$ & 1,290732 \\
\hline 4 & 0,151558 & 0,169376 & 0,342358 & 0,627438 & 0,015903 & $3,05 \mathrm{E}-05$ & $1,69 \mathrm{E}+11$ & $1,69 \mathrm{E}+11$ & $2,32 \mathrm{E}+11$ & $1 \mathrm{E}-05$ & 0,009 & 25,61739 & $1 \mathrm{E}-12$ & $1 \mathrm{E}-12$ & $1 \mathrm{E}-12$ & 1,290732 \\
\hline 5 & $1 \mathrm{E}-12$ & $1 \mathrm{E}-12$ & $1 \mathrm{E}-12$ & $1 \mathrm{E}-12$ & 0,057892 & 0,000121 & $1 \mathrm{E}-12$ & $1 \mathrm{E}-12$ & $1 \mathrm{E}-12$ & $1 \mathrm{E}-05$ & 0,009 & 25,61739 & $1 \mathrm{E}-12$ & $1 \mathrm{E}-12$ & $1 \mathrm{E}-12$ & $1,03 \mathrm{E}+08$ \\
\hline 6 & 0,448612 & 0,501354 & 1,013409 & 1,85715 & $6,22 \mathrm{E}+08$ & 0,000211 & $5,5 \mathrm{E}+32$ & $8,65 E+32$ & $2,78 \mathrm{E}+34$ & $1 \mathrm{E}-05$ & 0,009 & 25,61739 & $1 \mathrm{E}-12$ & $1 \mathrm{E}-12$ & $1 \mathrm{E}-12$ & $1,01 \mathrm{E}+08$ \\
\hline 7 & 0,448612 & 0,501354 & 1,013409 & 1,85715 & $6,22 \mathrm{E}+08$ & 0,000211 & 66749429 & $1,05 \mathrm{E}+08$ & $3,37 \mathrm{E}+09$ & $1 \mathrm{E}-05$ & 0,009 & 25,61739 & $1 \mathrm{E}-12$ & $1 \mathrm{E}-12$ & $1 \mathrm{E}-12$ & $1,01 \mathrm{E}+08$ \\
\hline 8 & $1 \mathrm{E}-12$ & $1 \mathrm{E}-12$ & $1 \mathrm{E}-12$ & $1 \mathrm{E}-12$ & $1 \mathrm{E}-12$ & $1 \mathrm{E}-12$ & $1,03 \mathrm{E}-19$ & $1,59 \mathrm{E}-19$ & $1 \mathrm{E}-12$ & $1 \mathrm{E}-12$ & 0,009 & 25,61739 & $1 \mathrm{E}-12$ & $1 \mathrm{E}-12$ & $1 \mathrm{E}-12$ & $1 \mathrm{E}-12$ \\
\hline 9 & 0,448612 & 0,501356 & 1,013411 & 1,857043 & $6,18 \mathrm{E}+08$ & $9,04 \mathrm{E}-05$ & $5,36 \mathrm{E}+32$ & $8,44 \mathrm{E}+32$ & $2,76 \mathrm{E}+34$ & $1 \mathrm{E}-12$ & 0,009 & 25,61739 & $1 \mathrm{E}-12$ & $1 \mathrm{E}-12$ & $1 \mathrm{E}-12$ & $1 \mathrm{E}-12$ \\
\hline 10 & 0,448612 & 0,501356 & 1,013411 & 1,857043 & $6,18 \mathrm{E}+08$ & $9,04 \mathrm{E}-05$ & 65117536 & $1,02 \mathrm{E}+08$ & $3,35 \mathrm{E}+09$ & $1 \mathrm{E}-12$ & 0,009 & 25,61739 & $1 \mathrm{E}-12$ & $1 \mathrm{E}-12$ & $1 \mathrm{E}-12$ & $1 \mathrm{E}-12$ \\
\hline 11 & $1 \mathrm{E}-12$ & $1 \mathrm{E}-12$ & $1 \mathrm{E}-12$ & $1 \mathrm{E}-12$ & $1 \mathrm{E}-12$ & $1 \mathrm{E}-12$ & $1,02 \mathrm{E}-19$ & 1,57E-19 & $1 \mathrm{E}-12$ & $1 \mathrm{E}-12$ & 0,009 & 25,61739 & $1 \mathrm{E}-12$ & $1 \mathrm{E}-12$ & $1 \mathrm{E}-12$ & $1 \mathrm{E}-12$ \\
\hline 12 & 0,448612 & 0,501357 & 1,013414 & 1,856929 & $6,14 \mathrm{E}+08$ & $9,04 \mathrm{E}-05$ & $5,23 \mathrm{E}+32$ & $8,23 \mathrm{E}+32$ & $2,74 \mathrm{E}+34$ & $1 \mathrm{E}-12$ & 0,009 & 25,61739 & $1 \mathrm{E}-12$ & $1 \mathrm{E}-12$ & $1 \mathrm{E}-12$ & $1 \mathrm{E}-12$ \\
\hline 13 & 0,448612 & 0,501357 & 1,013414 & 1,856929 & $6,14 \mathrm{E}+08$ & $9,04 \mathrm{E}-05$ & 63525615 & $1 \mathrm{E}+08$ & $3,33 \mathrm{E}+09$ & $1 \mathrm{E}-12$ & 0,009 & 25,61739 & $1 \mathrm{E}-12$ & $1 \mathrm{E}-12$ & $1 \mathrm{E}-12$ & $1 \mathrm{E}-12$ \\
\hline 14 & $1 \mathrm{E}-12$ & $1 \mathrm{E}-12$ & $1 \mathrm{E}-12$ & $1 \mathrm{E}-12$ & $1 \mathrm{E}-12$ & $1 \mathrm{E}-12$ & 1,01E-19 & $1,55 \mathrm{E}-19$ & $1 \mathrm{E}-12$ & $1 \mathrm{E}-12$ & 0,009 & 25,61739 & $1 \mathrm{E}-12$ & $1 \mathrm{E}-12$ & $1 \mathrm{E}-12$ & $1 \mathrm{E}-12$ \\
\hline 15 & 0,448612 & 0,501358 & 1,013416 & 1,856815 & $6,1 \mathrm{E}+08$ & $9,04 \mathrm{E}-05$ & $5,1 \mathrm{E}+32$ & $8,03 \mathrm{E}+32$ & $2,72 \mathrm{E}+34$ & $1 \mathrm{E}-12$ & 0,009 & 25,61739 & $1 \mathrm{E}-12$ & $1 \mathrm{E}-12$ & $1 \mathrm{E}-12$ & $1 \mathrm{E}-12$ \\
\hline 16 & 0,448612 & 0,501358 & 1,013416 & 1,856815 & $6,1 \mathrm{E}+08$ & $9,04 \mathrm{E}-05$ & 61972553 & 97579107 & $3,31 \mathrm{E}+09$ & $1 \mathrm{E}-12$ & 0,009 & 25,61739 & $1 \mathrm{E}-12$ & $1 \mathrm{E}-12$ & $1 \mathrm{E}-12$ & $1 \mathrm{E}-12$ \\
\hline 17 & $1 \mathrm{E}-12$ & $1 \mathrm{E}-12$ & $1 \mathrm{E}-12$ & $1 \mathrm{E}-12$ & $1 \mathrm{E}-12$ & $1 \mathrm{E}-12$ & $9,93 \mathrm{E}-20$ & $1,53 \mathrm{E}-19$ & $1 \mathrm{E}-12$ & $1 \mathrm{E}-12$ & 0,009 & 25,61739 & $1 \mathrm{E}-12$ & $1 \mathrm{E}-12$ & $1 \mathrm{E}-12$ & $1 \mathrm{E}-12$ \\
\hline 18 & 0,448612 & 0,50136 & 1,013419 & 1,856701 & $6,06 \mathrm{E}+08$ & $9,04 \mathrm{E}-05$ & $4,98 \mathrm{E}+32$ & $7,84 \mathrm{E}+32$ & $2,71 \mathrm{E}+34$ & $1 \mathrm{E}-12$ & 0,009 & 25,61739 & $1 \mathrm{E}-12$ & $1 \mathrm{E}-12$ & $1 \mathrm{E}-12$ & $1 \mathrm{E}-12$ \\
\hline 19 & 0,448612 & 0,50136 & 1,013419 & 1,856701 & $6,06 \mathrm{E}+08$ & 9,04E-05 & 60457451 & 95215661 & $3,29 \mathrm{E}+09$ & $1 \mathrm{E}-12$ & 0,009 & 25,61739 & $1 \mathrm{E}-12$ & $1 \mathrm{E}-12$ & $1 \mathrm{E}-12$ & $1 \mathrm{E}-12$ \\
\hline 20 & $1 \mathrm{E}-12$ & $1 \mathrm{E}-12$ & $1 \mathrm{E}-12$ & $1 \mathrm{E}-12$ & $1 \mathrm{E}-12$ & $1 \mathrm{E}-12$ & $9,82 \mathrm{E}-20$ & $1,52 \mathrm{E}-19$ & $1 \mathrm{E}-12$ & $1 \mathrm{E}-12$ & 0,009 & 25,61739 & $1 \mathrm{E}-12$ & $1 \mathrm{E}-12$ & $1 \mathrm{E}-12$ & $1 \mathrm{E}-12$ \\
\hline 799.996 & 0,004968 & 0,040668 & 0,066903 & 0,097306 & 0,058816 & 1,23E-07 & $3,37 \mathrm{E}-07$ & 0,0167 & 0,209526 & $1 \mathrm{E}-12$ & 0,009 & 25,61739 & $1 \mathrm{E}-12$ & $5,13 \mathrm{E}-13$ & $5,13 \mathrm{E}-13$ & 0,209582 \\
\hline 799.997 & $1 \mathrm{E}-12$ & $1 \mathrm{E}-12$ & $1 \mathrm{E}-12$ & $1 \mathrm{E}-12$ & $1 \mathrm{E}-12$ & $1 \mathrm{E}-12$ & $1 \mathrm{E}-12$ & $1,32 \mathrm{E}-08$ & $1 \mathrm{E}-12$ & $1 \mathrm{E}-12$ & 0,009 & 25,61739 & $1 \mathrm{E}-12$ & $5,13 \mathrm{E}-13$ & $5,13 \mathrm{E}-13$ & $1 \mathrm{E}-12$ \\
\hline 799.998 & 0,004967 & 0,040666 & 0,0669 & 0,097301 & 0,058137 & $1,22 \mathrm{E}-07$ & $3,74 \mathrm{E}+16$ & $2,2 \mathrm{E}+23$ & $2,6 \mathrm{E}+24$ & $1 \mathrm{E}-12$ & 0,009 & 25,61739 & $1 \mathrm{E}-12$ & $5,13 \mathrm{E}-13$ & $5,13 \mathrm{E}-13$ & 0,209636 \\
\hline 799.999 & 0,004967 & 0,040666 & 0,0669 & 0,097301 & 0,058137 & $1,22 \mathrm{E}-07$ & 2,98E-09 & 0,017521 & 0,20683 & $1 \mathrm{E}-12$ & 0,009 & 25,61739 & $1 \mathrm{E}-12$ & $5,13 \mathrm{E}-13$ & $5,13 \mathrm{E}-13$ & 0,209636 \\
\hline 800.000 & $1 \mathrm{E}-12$ & $1 \mathrm{E}-12$ & $1 \mathrm{E}-12$ & $1 \mathrm{E}-12$ & $1 \mathrm{E}-12$ & $1 \mathrm{E}-12$ & $1 \mathrm{E}-12$ & $1,32 \mathrm{E}-08$ & $1 \mathrm{E}-12$ & $1 \mathrm{E}-12$ & 0,009 & 25,61739 & $1 \mathrm{E}-12$ & 5,13E-13 & 5,13E-13 & $1 \mathrm{E}-12$ \\
\hline
\end{tabular}

Fonte: Própria autoria. 
Para algumas espécies (16 primeiras listadas), os efeitos das reações químicas surgem nos instantes inicias (por volta da $3^{\mathrm{a}}$ iteração nas simulações com 80, 800, 8.000 e 80.000 iterações, como mostram as Tabelas 21 a 24, e da $5^{\text {a }}$ iteração nas simulações com 800.000 iterações conforme a Tabela 25). Justamente a partir desse ponto começam as instabilidades numéricas, particularmente com relação às concentrações das espécies solúveis hidrogênio, metano e carbono inorgânico.

No perfil das concentrações simuladas por Danielsson (2014) para estas espécies, vêse que o aumento na concentração ocorre em intervalo de tempo pequeno (derivada positiva) como também se vê a rápida mudança na derivada da concentração com relação ao tempo. Neste sentido, acredita-se que o simulador não teve sensibilidade numérica suficiente para tal variação e divergiu.

Ao avaliar o comportamento posterior destas concentrações, nota-se que o simulador procura retomar o padrão simulado por Danielsson (2014). A Tabela 25 mostra que ao final de 800.000 iterações a concentração de $\mathrm{H}_{2}$ solúvel chega a valores muito baixos, o que foi igualmente simulado por Danielsson (2014) e Rosen e Jeppsson (2006). As concentrações de metano e carbono inorgânico também atingiram valores coerentes ao final do processo. Para demais espécies listadas na segunda parte de cada tabela, também ficou evidente certa instabilidade nas iterações iniciais, na maioria das vezes superada pelo simulador posto que depois foi capaz de convergir para valores condizentes.

Por exemplo, as concentrações de bicarbonato $\left(\mathrm{HCO}_{3}{ }^{-}\right)$inicialmente apresentaram desvios significativos. Neste caso recomenda-se utilizar equações algébricas para representar a concentração de bicarbonato na fase líquida ao invés de equações dinâmicas, na medida em que são mais simples e podem evitar erros e/ou instabilidades numéricas (IWA, 2002). Queen (2006) empregou equações algébricas para modelar as concentrações de bicarbonato $\left(\mathrm{HCO}_{3}{ }^{-}\right)$ e gás carbônico $\left(\mathrm{CO}_{2}\right)$ solúveis como também empregou valores fixos para o $\mathrm{pH}$, alcançando assim convergência nas simulações.

Para 5 espécies solúveis, a Figura 23 a seguir compara as simulações com 80, 800, 8.000, 80.000 e 800.000 iterações. Nota-se que a partir de 80.000 iterações, o passo de tempo torna-se suficientemente pequeno para que o simulador tenha a devida sensibilidade numérica para convergência da solução. 
Figura 23 - Comparação de diferentes números de iterações $(n)$ para as concentrações das espécies solúveis: açúcares, valerato, butirato, propionato e acetato, na simulação 2
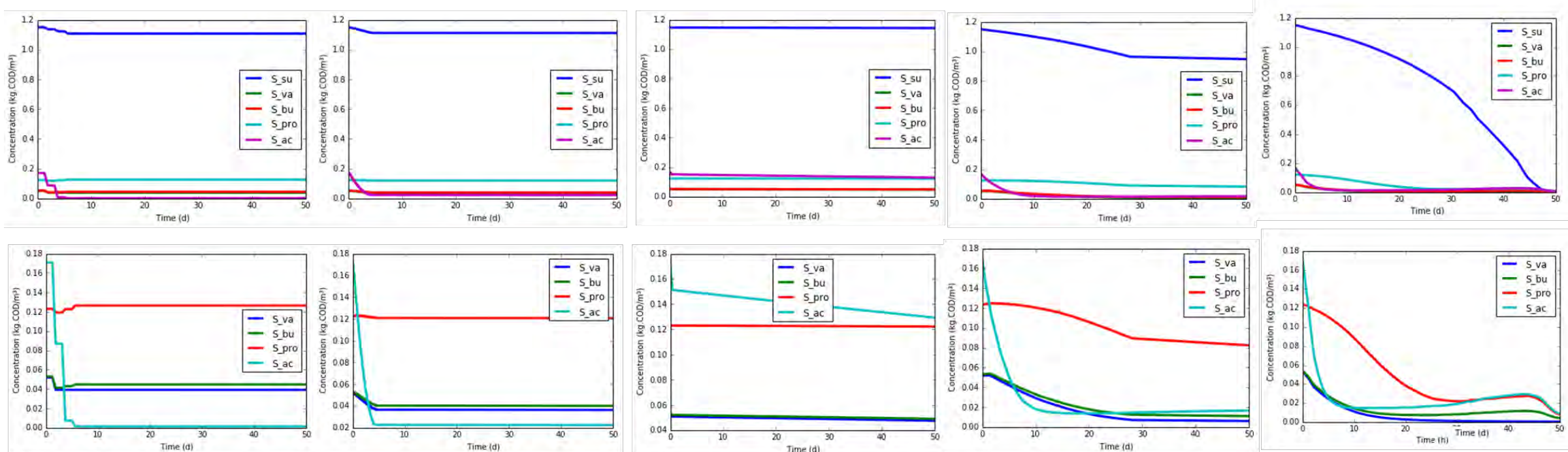

$$
n=80
$$

$n=800$

$n=8.000$

$n=80.000$

$n=800.000$

Fonte: Própria autoria. 


\subsection{Simulação 3: Simulação completa ADM1}

Nesta simulação foram consideradas todas as rotas metabólicas do modelo ADM1 incluindo, portanto, as etapas de hidrólise e a degradação de aminoácidos e ácidos graxos de cadeia longa, tal como adotado por Rosen e Jeppsson (2006) e Danielsson (2014). Seguindo os valores utilizados por Danielsson (2014), a Tabela 26 mostra as variáveis adotadas na alimentação assim como as estimativas iniciais.

A Tabela 25 também compara concentrações obtidas por meio desta simulação contra resultados obtidos por Rosen e Jeppsson (2006) e Danielsson (2014). Realizando 800.000 iterações, esta simulação considerou um período de 50 dias, tal como por Danielsson (2014). Os perfis de concentração simulados para cada espécie considerada ao longo dos 50 dias de tratamento são apresentados nas Figuras 24 a 28.

As concentrações de açúcares e aminoácidos, Figuras 24(a)-(b), apresentaram perfis muito semelhantes, assim como observado nas simulações de Danielsson (2014). Já os perfis das concentrações de ácidos graxos e propionato, Figuras 24(c) e (f) foram diferentes dos encontrados por Danielsson (2014). As concentrações de valerato e butirato, Figuras 24(d) e (e) tiveram comportamento semelhante entre si, porém diferente das concentrações de ácidos graxos e propionato. Estas últimas tiveram comportamento parecido com a variação da concentração de acetato.

A concentração de metano solúvel, Figura 24 (h), também apresentou comportamento semelhante àquele simulado por Danielsson (2014), atingindo estado estacionário por volta de 15 dias de tratamento. A concentração de carbono inorgânico, Figura 25(a), apresentou um decréscimo muito rápido, atingindo o estado estacionário em poucas horas, opostamente à simulação de Danielsson (2014).

A concentração de material particulado e das biomassas consumidoras de carboidratos, proteínas e lipídeos, Figuras 25(c)- (f), apresentaram perfis de concentração semelhantes. A concentração de material particulado e biomassa de carboidratos atingiram estado estacionário entre 15 e 20 dias, de forma similar ao trabalho utilizado como comparação. As biomassas consumidoras de açúcares e aminoácidos, Figuras 25(g)-(h), apresentaram perfis semelhantes aos de Danielsson (2014). Por outro lado, os perfis das concentrações de biomassas de ácidos graxos, valerato e butirato (c4), propionato e acetato, Figura 26(a)-(d), foram diferentes, apresentando uma diminuição cujo início ocorre entre 15 e 20 dias. 
Tabela 26 - Comparação entre as simulações feitas (1) neste trabalho e (2) Rosen e Jeppsson (2006) e Danielsson (2014) para as concentrações das diferentes espécies após 50 dias de tratamento

\begin{tabular}{|c|c|c|c|c|c|c|c|}
\hline \multirow[b]{2}{*}{ Espécie } & \multirow[b]{2}{*}{ Unidade } & \multicolumn{3}{|c|}{ (1) Simulação 3} & \multicolumn{2}{|c|}{ (2) Danielsson (2014) } & \multirow{2}{*}{$\begin{array}{c}\text { Rosen e Jeppsson (2006) } \\
\text { Após } 50 \text { dias }\end{array}$} \\
\hline & & Alimentação & Estimativa inicial & Após 50 dias & Alimentação & Estimativa inicial & \\
\hline$S_{\mathrm{su}}$ & $\mathrm{kg}-\mathrm{DQO} \cdot \mathrm{m}^{-3}$ & 0,01 & 0,009 & 0,1138688505742 & 0,01 & 0,009 & 0,0119548297170 \\
\hline$s_{\mathrm{aa}}$ & $\mathrm{kg}$-DQO. $\mathrm{m}^{-3}$ & 0,001 & 0,0009 & 0,2516255262294 & 0,001 & 0,0009 & 0,0053147401716 \\
\hline$s_{\mathrm{fa}}$ & kg-DQO.m ${ }^{-3}$ & 0,001 & 0,0009 & 0,0475136399519 & 0,001 & 0,0009 & 0,0986214009308 \\
\hline$s_{\mathrm{va}}$ & kg-DQO. $\mathrm{m}^{-3}$ & 0,001 & 0,0009 & 5,9326967625018 & 0,001 & 0,0009 & 0,0116250064639 \\
\hline${ }^{s} \mathrm{bu}$ & $\mathrm{kg}-\mathrm{DQO} . \mathrm{m}^{-3}$ & 0,001 & 0,0009 & 0,3062889314185 & 0,001 & 0,0009 & 0,0132507296663 \\
\hline$s_{\text {pro }}$ & kg-DQO. $\mathrm{m}^{-3}$ & 0,001 & 0,0009 & 3,0705978314349 & 0,001 & 0,0009 & 0,0157836662845 \\
\hline$s_{\mathrm{ac}}$ & $\mathrm{kg}-\mathrm{DQO} . \mathrm{m}^{-3}$ & 0,001 & 0,00009 & 8,1495565734973 & 0,001 & 0,00009 & 0,1976297169376 \\
\hline$s_{\mathrm{h} 2}$ & kg-DQO.m ${ }^{-3}$ & $1,00 \mathrm{E}-08$ & 2,3594E-09 & 0,0000000000010 & $1,00 \mathrm{E}-08$ & 2,3594E-09 & 0,0000002359451 \\
\hline$s_{\operatorname{ch} 4}$ & kg-DQO. $\mathrm{m}^{-3}$ & $1,00 \mathrm{E}-05$ & 2,3594E-05 & 0,2207547577212 & $1,00 \mathrm{E}-05$ & 2,3594E-05 & 0,0550887764460 \\
\hline${ }^{S_{\mathrm{IC}}}$ & kmol-C.m ${ }^{-3}$ & 0,04 & 0,039 & 0,0000000000010 & 0,04 & 0,039 & 0,1526778706263 \\
\hline${ }^{s} \mathrm{IN}$ & kmol-N.m ${ }^{-3}$ & 0,01 & 0,1302 & 0,0000336384591 & 0,01 & 0,1302 & 0,1302298158037 \\
\hline$x_{\mathrm{c}}$ & kg-DQO. $\mathrm{m}^{-3}$ & 2 & 0,3087 & 1,0691998996675 & 2 & 0,3087 & 0,3086976637215 \\
\hline$x_{\mathrm{ch}}$ & kg-DQO.m ${ }^{-3}$ & 5 & 0,028 & 0,0355148839964 & 5 & 0,028 & 0,0279472404350 \\
\hline${ }^{X} \mathrm{pr}$ & $\mathrm{kg}-\mathrm{DQO} . \mathrm{m}^{-3}$ & 20 & 0,1025 & 0,1101417496681 & 20 & 0,1025 & 0,1025741061067 \\
\hline$x_{\mathrm{li}}$ & kg-DQO. $\mathrm{m}^{-3}$ & 5 & 0,0295 & 0,0408345150493 & 5 & 0,0295 & 0,0294830497073 \\
\hline$x_{\mathrm{su}}$ & kg-DQO. $\mathrm{m}^{-3}$ & 0 & 0,4202 & 0,5221088389504 & 0 & 0,4202 & 0,4201659824546 \\
\hline$x_{\mathrm{aa}}$ & kg-DQO. $\mathrm{m}^{-3}$ & 0,01 & 1,1792 & 1,2579748759466 & 0,01 & 1,1792 & 1,1791717989237 \\
\hline$x_{\mathrm{fa}}$ & $\mathrm{kg}-\mathrm{DQO} \cdot \mathrm{m}^{-3}$ & 0,01 & 0,243 & 0,0549399264320 & 0,01 & 0,243 & 0,2430353447194 \\
\hline$x_{\mathrm{c} 4}$ & kg-DQO.m ${ }^{-3}$ & 0,01 & 0,4319 & 0,4438487595909 & 0,01 & 0,4319 & 0,4319211056360 \\
\hline${ }^{x}$ pr & $\mathrm{kg}-\mathrm{DQO} . \mathrm{m}^{-3}$ & 0,01 & 0,1373 & 0,0523582738626 & 0,01 & 0,1373 & 0,1373059089340 \\
\hline$x_{\mathrm{ac}}$ & kg-DQO. $\mathrm{m}^{-3}$ & 0,01 & 0,7606 & 0,3220234474500 & 0,01 & 0,7606 & 0,7605626583132 \\
\hline
\end{tabular}




\begin{tabular}{|c|c|c|c|c|c|c|c|}
\hline$x_{\mathrm{h} 2}$ & $\mathrm{~kg}-\mathrm{DQO} \cdot \mathrm{m}^{-3}$ & 0,01 & 0,317 & 21,7269291191210 & 0,01 & 0,317 & 0,3170229533613 \\
\hline$s_{\text {cat }}$ & kg-DQO.m ${ }^{-3}$ & 0,04 & 0,04 & 0,0400000000000 & 0,04 & 0,04 & 0,0400000000000 \\
\hline$s_{\text {an }}$ & kg-DQO.m ${ }^{-3}$ & 0,02 & 0,02 & 25,2988507231628 & 0,02 & 0,02 & 0,0200000000000 \\
\hline$S_{\text {vam }}$ & $\mathrm{kg}-\mathrm{DQO} \cdot \mathrm{m}^{-3}$ & $\mathrm{X}$ & 0,0116 & 2,1708064751301 & $\mathrm{x}$ & 0,0116 & 0,0115962470725 \\
\hline${ }^{s}$ bum & $\mathrm{kg}-\mathrm{DQO} \cdot \mathrm{m}^{-3}$ & $\mathrm{X}$ & 0,0132 & 2,8973210834890 & $\mathrm{x}$ & 0,0132 & 0,0132208262485 \\
\hline Sprom & $\mathrm{kg}-\mathrm{DQO} \cdot \mathrm{m}^{-3}$ & $\mathrm{X}$ & 0,0157 & 25,2988507231628 & $\mathrm{x}$ & 0,0157 & 0,0157427831916 \\
\hline$s_{\mathrm{acm}}$ & $\mathrm{kg}-\mathrm{DQO} \cdot \mathrm{m}^{-3}$ & $\mathrm{X}$ & 0,1972 & 88,5140656961522 & $\mathrm{x}$ & 0,1972 & 0,1972411554366 \\
\hline$s_{\text {nh3 }}$ & $\mathrm{kmol}-\mathrm{N} \cdot \mathrm{m}^{-3}$ & $\mathrm{X}$ & 0,0041 & 0,0000000241627 & $\mathrm{x}$ & 0,0041 & 0,0040909284584 \\
\hline$s_{\text {gas, } \mathrm{h} 2}$ & kg-DQO. $\mathrm{m}^{-3}$ & $\mathrm{X}$ & $1,023 \mathrm{E}-05$ & 0,0000674087925 & $\mathrm{x}$ & $1,023 \mathrm{E}-05$ & 0,0000102410356 \\
\hline$s_{\text {gas, ch4 }}$ & $\mathrm{kg}-\mathrm{DQO} \cdot \mathrm{m}^{-3}$ & $\mathrm{X}$ & 1,6213 & 3,3008253135167 & $\mathrm{x}$ & 1,6213 & 1,6256072099814 \\
\hline gas,co2 & kmol-C.m ${ }^{-3}$ & $\mathrm{X}$ & 0,01410 & 0,0000011130944 & $\mathrm{x}$ & 0,01410 & 0,0141505346784 \\
\hline${ }^{s} \mathbf{I}$ & $\mathrm{kg}-\mathrm{DQO} \cdot \mathrm{m}^{-3}$ & 0,02 & 0,00900 & 0,9575947842142 & 0,02 & 0,00900 & 0,3286976637215 \\
\hline${ }^{x}$ lac,o & d_X_lac_o_dts & 0 & 0 & 0,0000000000000 & 0 & 0 & Não simulado \\
\hline$s_{\mathrm{ca}}$ & kmole. $\mathrm{m}^{-3}$ & 0 & 0 & 0,0000000000001 & 0 & 0 & Não simulado \\
\hline${ }^{s} \mathrm{DQO}$ & S_DQO_totals & 0,001 & 2,7 & 118,8790447771690 & 0,001 & 2,7 & Não simulado \\
\hline
\end{tabular}

X: concentração destas espécies na alimentação é considerada nula, entretanto não é necessário declaração dos valores na alimentação. Fonte: Própria autoria. 
Figura 24 - Perfil de concentração das espécies solúveis: açúcares (a), aminoácidos (b), ácidos graxos (c), valerato (d), butirato (e), propionato (f), acetato (g) e metano (h)
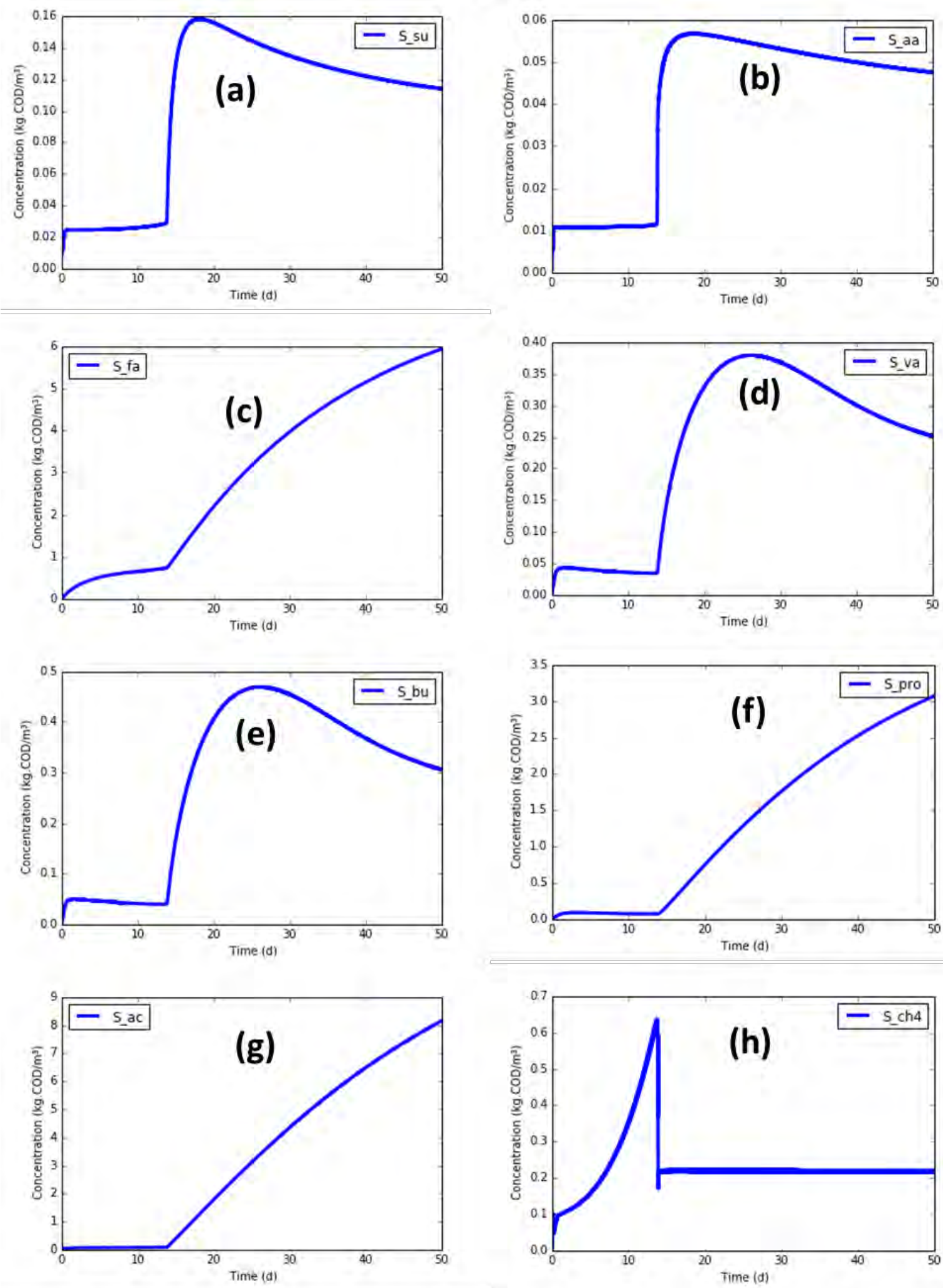

Fonte: Própria autoria. 
Figura 25 - Perfil de concentração das espécies: carbono inorgânico (a), nitrogênio inorgânico (b), compósitos (c) e biomassa de: carboidratos (d), proteínas (e), lipídeos (f), açúcares (g) e aminoácidos (h)
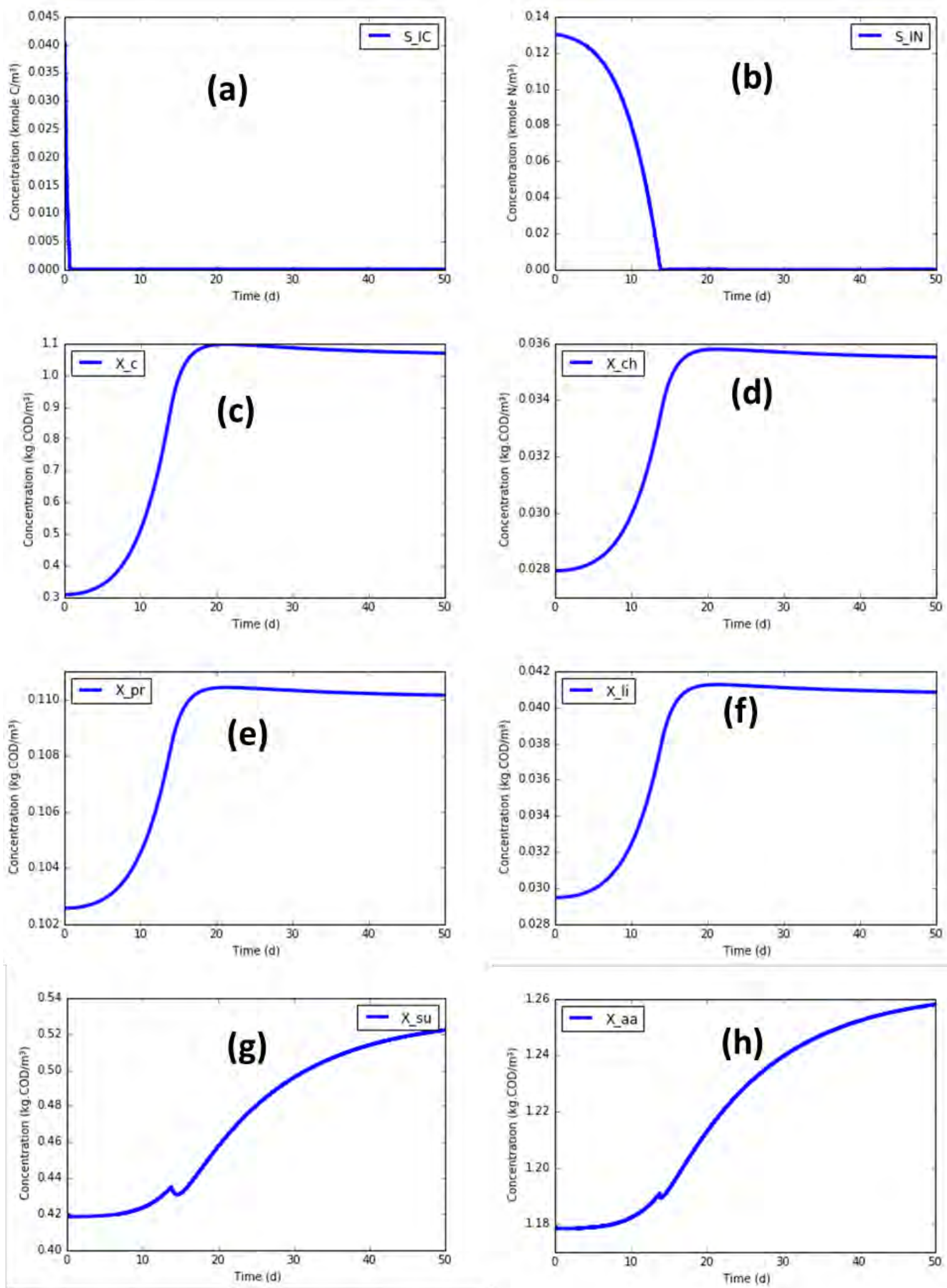

Fonte: Própria autoria. 
Figura 26 - Perfil de concentração das biomassa de: ácidos graxos (a), butirato e valerato (b), propionato (c) acetato (d), hidrogênio (e) e cátions (f), ânions (g) e ácido valérico (h) solúveis
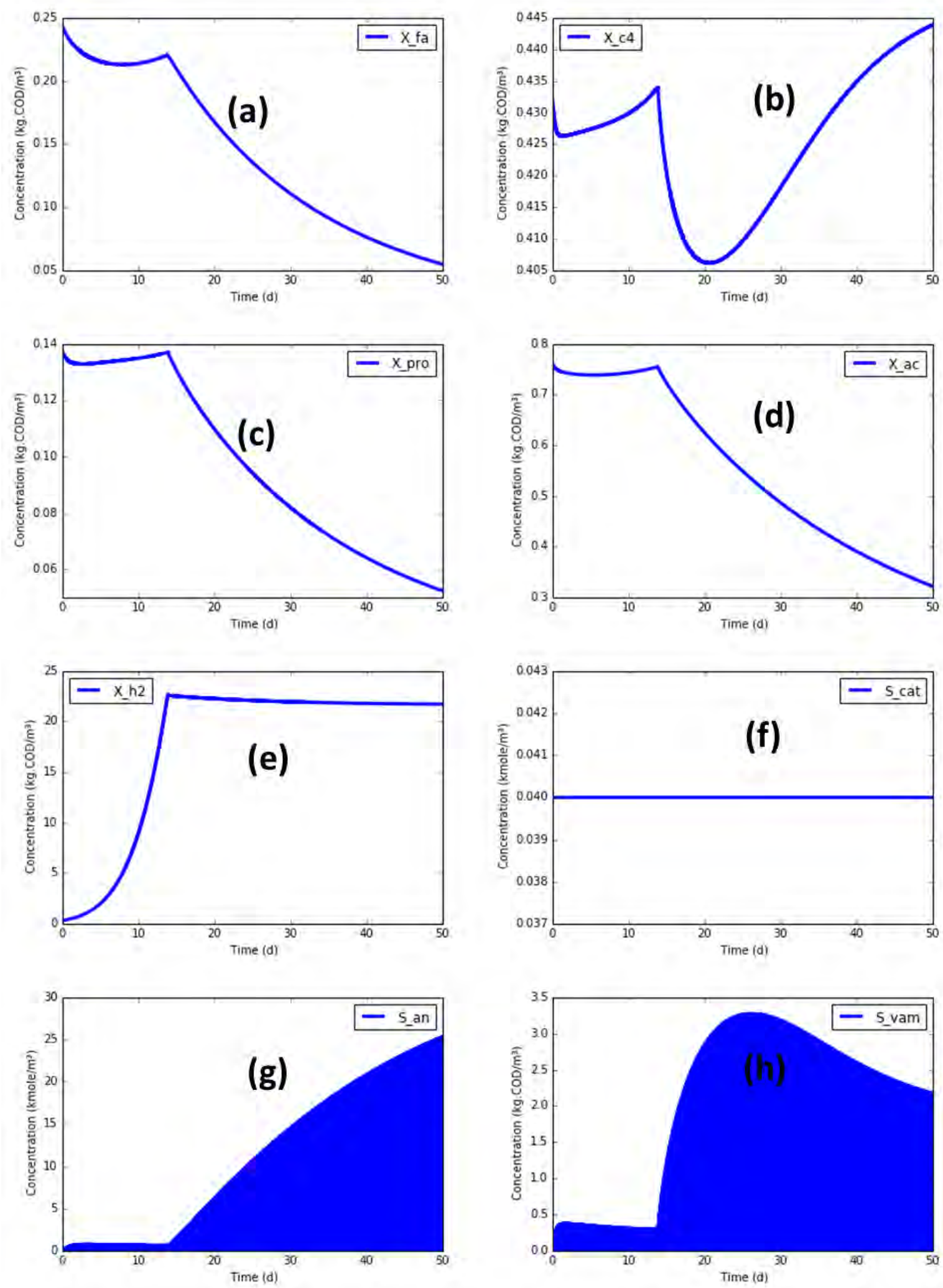

Fonte: Própria autoria. 
Figura 27 - Perfil de concentração das espécies: ácido butírico (a), ácido propiônico (b), ácido acético (c), bicarbonato (d), $\mathrm{NH}_{3}$ (e), $\mathrm{H}_{2}$ (f), $\mathrm{CH}_{4}$ (g) e $\mathrm{CO}_{2}$ (h)
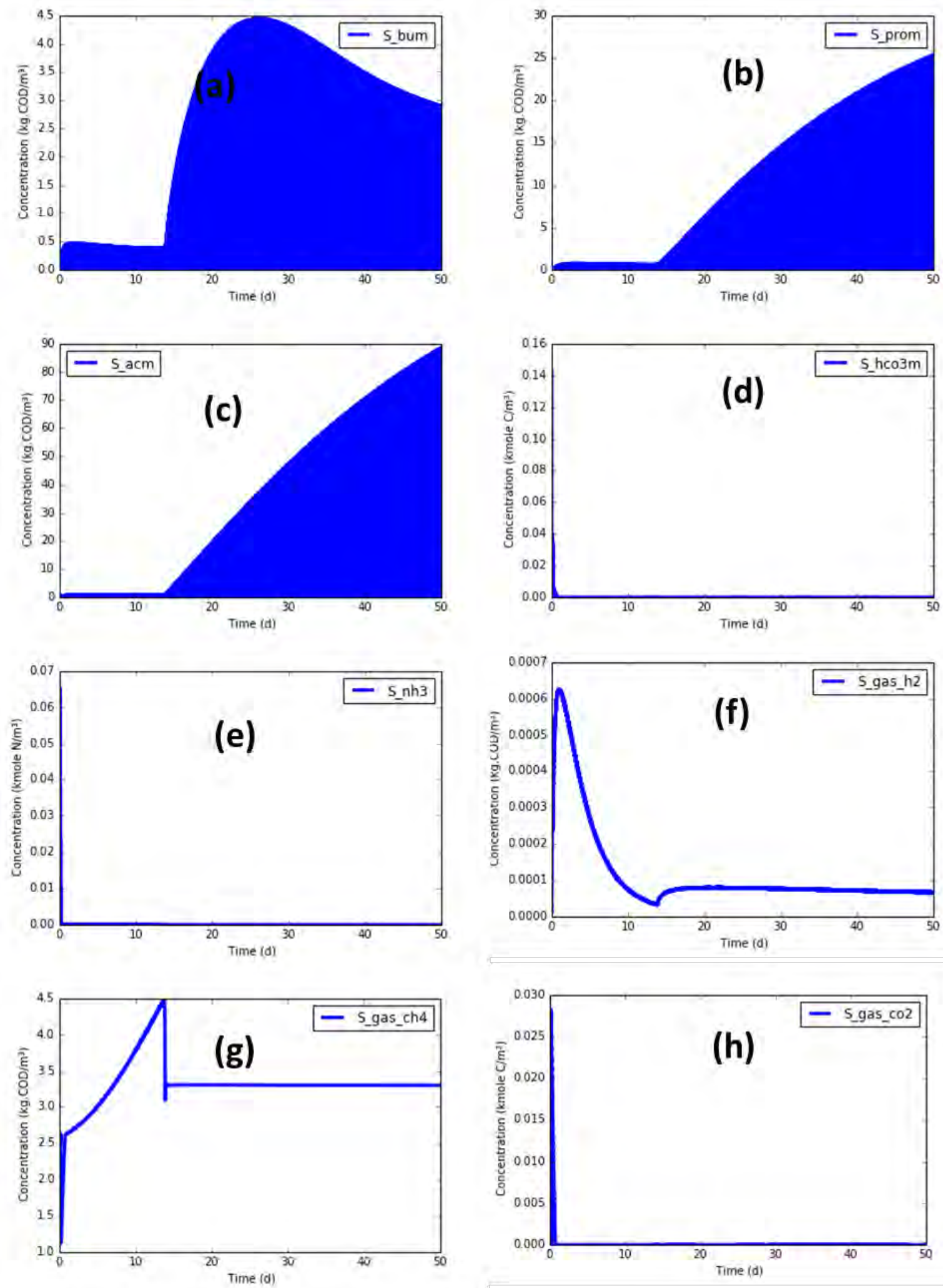

Fonte: Própria autoria. 
Figura 28 - Perfil de concentração das espécies: inertes particulados (a), inertes solúveis (b), cálcio (c), lactato (d), lactato da fermentação (e), lactato da oxidação (f) e DQO (g)
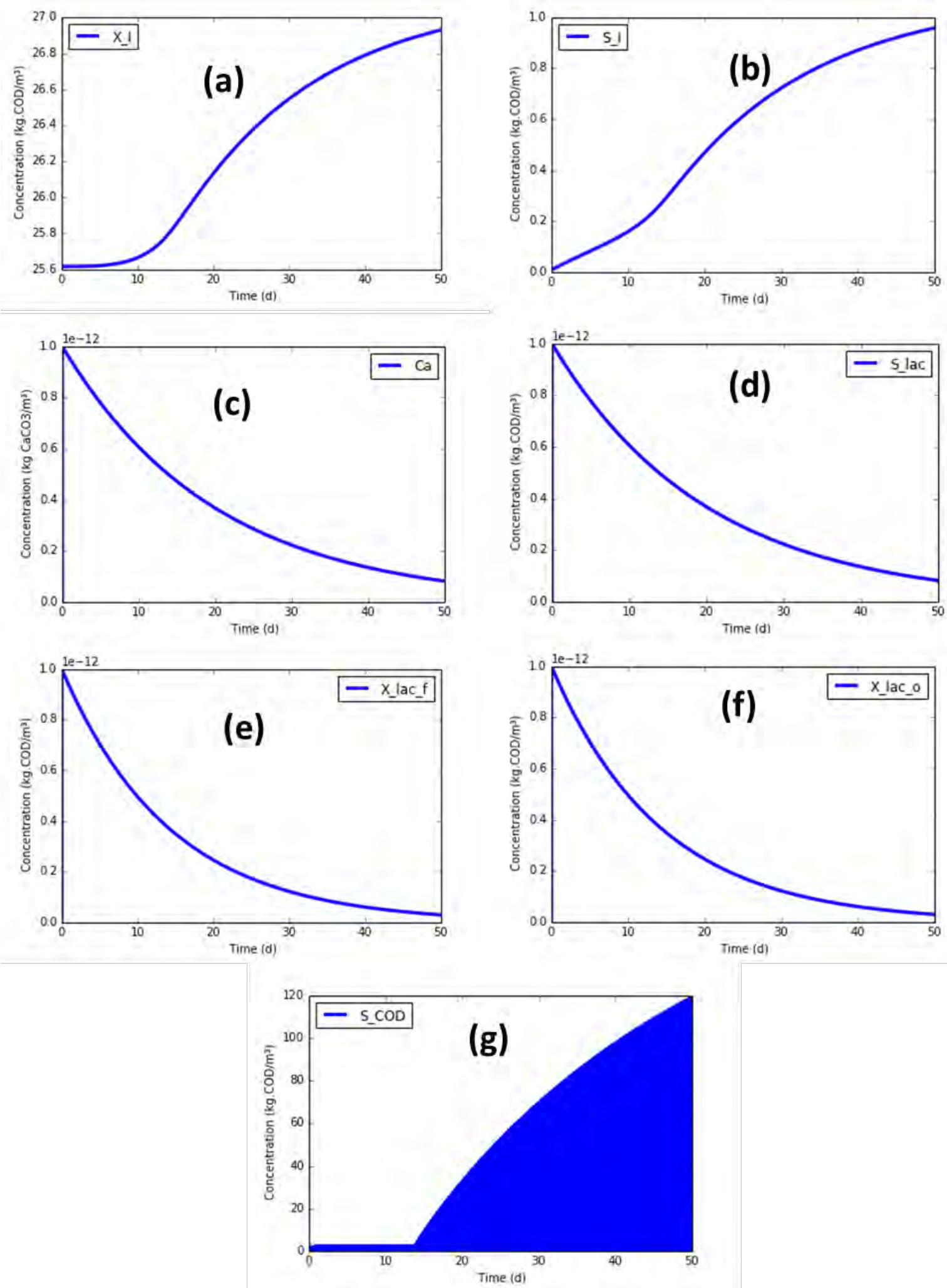

Fonte: Própria autoria. 
Os perfis dos ácidos valérico, butírico, propiônico e acético, Figura 26(h) e Figuras 27(a)-(c), apresentaram valores instáveis ao longo desta simulação. Porém, os perfis das curvas das concentrações de ácido valérico e butírico foram semelhantes aos encontrados por Danielsson (2014). Conforme mostra as Figura 27(d)-(e), as concentrações de bicarbonato e amônia se mantiveram quase nulas nesta simulação, o que precisa ser melhor avaliado.

$\mathrm{Na}$ fase gasosa, as concentrações de $\mathrm{CH}_{4}$ e $\mathrm{H}_{2}$ foram adequadamente simuladas, apresentando perfis de variação coerentes, Figuras 27(f)-(g). Já a concentração de $\mathrm{CO}_{2}$, Figura 27(h), precisa ser mais bem avaliada pois teve comportamento semelhante ao do bicarbonato e amônia, possivelmente influenciado pelas variações destas concentrações.

Pela análise geral das concentrações ao longo de 50 dias, observa-se que durante aproximadamente 24 dias o processo de hidrólise é mais acentuado, ocorrendo variações significativas nas concentrações de carboidratos, proteínas e lipídeos. Após cerca de 15 a 20 dias (quando se atinge o estado estacionário para maioria destes componentes), o sistema reage rapidamente na acidogênese, afetando drasticamente as concentrações de ácidos graxos, aminoácidos e açúcares da fase líquida. Entre o $8^{\circ}$ e o $15^{\circ}$ dia de tratamento, tem-se o ápice de etapa de metanogênese e por volta de 15 dias estabilizam-se as concentrações de metano e hidrogênio e da biomassa consumidora de hidrogênio.

\subsection{Tempo de processamento}

Os tempos de CPU para cada simulação estão apresentados na Tabela 27 abaixo.

Tabela 27 - Tempo de CPU aproximado em função de cada número de iterações (n)

\begin{tabular}{cccccc}
\hline Parâmetros adotados & $\mathbf{8 0}$ & $\mathbf{8 0 0}$ & $\mathbf{8 . 0 0 0}$ & $\mathbf{8 0 . 0 0 0}$ & $\mathbf{8 0 0 . 0 0 0}$ \\
\hline Simulação 1-A (50 dias) & $\mathrm{X}$ & $\mathrm{X}$ & $\mathrm{X}$ & $\mathrm{X}$ & $13 \mathrm{~min}$ \\
Simulação 1-B (5 dias) & $\mathrm{X}$ & $\mathrm{X}$ & $\mathrm{X}$ & $\mathrm{X}$ & $11 \mathrm{~min}$ \\
Simulação 1-C (50 dias) & $\mathrm{X}$ & $\mathrm{X}$ & $\mathrm{X}$ & $\mathrm{X}$ & $13 \mathrm{~min}$ \\
Simulação 2 (50 dias) & $11 \mathrm{~s}$ & $44 \mathrm{~s}$ & $6 \min 30 \mathrm{~s}$ & $1 \mathrm{~h} 05 \mathrm{~min}$ & $13 \mathrm{~h} 15 \mathrm{~min}$ \\
Simulação 3 (50 dias) & $\mathrm{X}$ & $\mathrm{X}$ & $\mathrm{X}$ & $\mathrm{X}$ & $22 \mathrm{~min}$ \\
\hline
\end{tabular}

Fonte: Própria autoria.

Os tempos de processamento variaram em função dos parâmetros escolhidos, tal como apontado por Danielsson (2014). Este autor associou a diferença no tempo de processamento 
do seu modelo implementado em MATLAB à complexidade dos parâmetros utilizados assim como a condição de aproximação destes ao estado estacionário.

Danielsson (2014) reportou tempo aproximado de 3 segundos para simulação em MATLAB, através do ODE-solver ode15s, que trabalha com passo variável dependendo de problemas de instabilidade numérica. O solver do Python utilizado neste trabalho (ODEint) não conseguiu ajustar corretamente todos os dados. Assim, uma melhoria a ser adotada para o simulador seria a implementação utilizando outro solver do próprio ambiente Python (por exemplo: complex ODE). 


\section{CONCLUSÃO}

Considerando as modificações propostas em Rosen e Jeppsson (2006) e adaptações feitas por Danielsson (2014), foi possível implementar um modelo computacional utilizando a linguagem de programação Python. Apesar das instabilidades numéricas verificadas em alguns cenários de operação, o simulador mostrou-se capaz de gerar concentrações com base no modelo implementado. A avaliação de diferentes parâmetros do modelo é de suma importância e afeta drasticamente o tempo de processamento do processo de simulação.

Implementado em uma linguagem disponível gratuitamente e com ampla utilização como Python, este simulador precisa ser aprimorado e pode ser utilizado como base para pesquisas futuras em modelagem e simulação de biorreatores, com especial interesse na vinhaça enquanto efluente agroindustrial. Desta forma, este trabalho beneficiará a comunidade científica de bioprocessos que trabalha com o modelo ADM1 de digestão anaeróbia para tratamento de efluentes agroindustriais. O simulador desenvolvido foi disponibilizado na forma de manual para o grupo de pesquisas AGROENERBIO - Energia e Simulação na Engenharia de Biossistemas e no Agronegócio. 


\section{DESENVOLVIMENTOS FUTUROS}

Para pesquisas futuras em modelagem e simulação de biorreatores existem inúmeras possibilidades de melhorias. Para o simulador desenvolvido neste trabalho foram apontadas algumas delas, detectadas pela autora deste trabalho e listadas a seguir:

- Realizar uma análise mais profunda do modelo e simulador;

- Avaliar a possibilidade de ajustes (como passo e tolerância interna) no solver ODEInt do Python para diminuir problemas numéricos (de instabilidade);

- Implementação do modelo utilizando outros solvers da biblioteca Scipy do Python, como "ode" que utiliza as rotinas VODE e ZVODE e/ou "complex_ode" que converte uma EDO de valor complexo para integral de valor real;

- Avaliar a simulação de outros parâmetros, condições operacionais ou mesmo a adoção de novos substratos de interesse podem ser testadas;

- Validação contra dados experimentais (além daqueles citados no presente trabalho);

- Comparar os resultados deste trabalho com outras plataformas de simulação;

- Ampliar o modelo para mais dimensões espaciais (uni, bi e tridimensionais);

- Gaden (2013) em sua pesquisa sugere a "rastreabilidade de partículas" que poderia proporcionar maior conhecimento sobre zonas de estagnação, perdas de volume causadas pela configuração das partículas e fornecer mais detalhes para controle do processo. Neste sentido, uma contribuição futura é a modelagem computacional do biorreator via método de Boltzmann em rede (LBM - lattice Boltzman method) (MOHAMAD, 2011).

Para facilitar a implementação de melhorias futuras ao simulador foi feito um manual (citado na conclusão deste trabalho) descrevendo detalhadamente o código de programação, relatando as dificuldades encontradas ao longo do seu desenvolvimento assim como suas implicações na execução do código. 


\section{REFERÊNCIAS}

ABBASI, T. et al. A brief history of anaerobic digestion and "Biogas". In: ABBASI, T.; TAUSEEF, S. M.; ABBASI, S. A. Biogas energy. New York: Springer-Verlag, 2012. p. 1123.

ANDREWS, J. F. A mathematical model for the continuous culture of microorganisms utilizing inhibitory substrates. Biotechnology and Bioengineering, Hoboken, v. 10, p. 707723, 1968.

ANGELIDAKI, I. I.; ELLEGAARD, L.; AHRING, B. K. A comprehensive model of anaerobic bioconversion of complex substrates to biogas. Biotechnology and

Bioengineering, New York, v. 63, n. 3, p. 363-372, 1999.

BATSTONE, D. J. et al. The IWA anaerobic digester model no.1 (ADM1). Water Science and Technology, Oxford, v. 45, n. 10, p. 65-73, 2002.

BATSTONE, D. J.; HERNANDEZ, J. L. A.; SCHMIDT, J. E. Hydraulics of laboratory and full-scale upflow anaerobic sludge blanket (UASB) reactors. Biotechnology and Bioengineering, Hoboken, v. 91, n. 3, p. 387-391, 2005.

BERNARD, J. Doing astronomy with python. Linux Journal, Houston, 19 Aug. 2015.

<http://www.linuxjournal.com/content/doing-astronomy-python>. Acesso em: 19 nov. 2016.

BOUBAKER, F.; RIDHA, B. C. Modelling of the mesophilic anaerobic co-digestion of olive mill wastewater with olive mill solid waste using anaerobic digestion model no.1 (ADM1).

Bioresource Technology, Amsterdam, v. 99, n. 14, p. 6565-6577, 2008.

BRITTON, A. et al. Pilot-scale struvite recovery from anaerobic digester supernatand at an enhanced biological phosphorus removal wastewater treatment plant. Journal of

Environmental Engineering and Science, London, v. 4, p. 265-277, 2005.

BROUWER. The Dynamic anaerobic reactor and integrated energy system (DARIES) model. 2010. Master Thesis, Clarkson University, 2010.

BUŁKOWSKA, K. et al. ADM1-based modeling of anaerobic codigestion of maize silage and cattle manure - calibration of parameters and model verification (part II). Archives of Environmental Protection, Zabrze, v. 41, n. 3, p. 20-27, 2015.

CHAI, Q. Modeling, estimation, and control of biological wastewater treatment plants. 2008. 184 p. Doctoral Thesis - Faculty of Technology, Telemark University College, Porsgrunn, 2008.

CHEN, Z. et al. Modelling of two-phase anaerobic process treating traditional Chinese medicine wastewater with the IWA anaerobic digestion model no.1. Bioresource Technology, Amsterdam, v. 100, n. 20, p. 4623-4631, 2009.

DANIELSSON, O. Modeling and simulation of anaerobic manure digestion into biogas. Master Thesis. Chalmers University of Technology, Sweden, Department of Physics \& Engineering Physics, 2014. 
DERBAL, K. et al. Application of the IWA ADM1 model to simulate anaerobic co-digestion of organic waste with activated sludge in mesophilic condition. Bioresource Technology, Amsterdam, v. 100, n. 4, p. 1539-1543, 2009.

DONOSO-BRAVO, A. et al. Modelling of an anaerobic plug-flow reactor: process analysis and evaluation approaches with non-ideal mixing considerations. Bioresource Technology, Amsterdam, v. 260, p. 95-104, 2018.

ESPAÑA-GAMBOA, E. et al. Vinasses: characterization and treatments. Waste Management \& Research, v.29, p. 1235-1250, 2011.

FARIA, A. B. B. et al. Evaluation of new alternatives in wastewater treatment plants based on dynamic modelling and lifecycle assessment (DM-LCA). Water Research, London, v. 1, n. 84, p. 99-111, 2015.

FERRAZ JÚNIOR, A. D. N. Digestão anaerobia da vinhaça da cana de açúcar em reator acidogênico de leito fixo seguido de reator metanogênico de manta de lodo. Tese de Doutorado. Escola de Engenharia de São Carlos, Universidade de São Paulo, São Carlos, 2013.

FORTUNATO, V. A. et al. Development of in-house lattice-Boltzmann simulator of bioreactors for wastewater treatment: basic concepts and initial results. Water Science and Technology, London, v. 77, p. 838-847, 2018.

GADEN, D. Modelling anaerobic digesters in three dimensions: integration of biochemistry with computational fluid dynamics. 2013. 421 p. Thesis (Doctor of Philosophy) - Faculty of Graduate Studies, University of Manitoba, Winnipeg, 2013.

GRADY JR., C. P. L. et al. Biological wastewater treatment. 3th ed. Boca Raton: CRC Press, 2011. 1022 p.

HOLMQVIST, A.; SELLBERG, A. A generic PAT software interface for on-line monitoring and control of chromatographic separation systems. Computer Aided Chemical Engineering, Amsterdam, v. 38. p. 811-816, 2016. 26th EUROPEAN SYMPOSIUM ON COMPUTER AIDED PROCESS ENGINEERING, 2016.

IFAK. SIMBA® 6.0. Guia de usuário. Magdeburg: Ifak System GmbH, 2009.

IWA TASK GROUP FOR MATHEMATICAL MODELLING OF ANAEROBIC DIGESTION PROCESSES. Anaerobic digestion model no 1 (ADM1). London: IWA Publishing, 2002. $77 \mathrm{p}$.

JACOB, S. M. Aplicação do modelo ADM1 na biodigestão anaeróbia da vinhaça para a produção de hidrogênio utilizando a plataforma EMSO. 2015. 89 f. Dissertação (Mestrado) - Instituto de Ciência e Tecnologia, Universidade Federal de Alfenas, Poços de Caldas, 2015. 
JORDAAN, E. M.; ACKERMAN, J.; CICEK, N. Phosphorus removal from anaerobically digested swine wastewater through struvite precipitation. Water Science and Technology, Oxford, v. 61, n. 12, p. 3228-3234, 2010.

JURADO, E.; GAVALA, H. N.; SKIADAS, I. ADM1-based modeling of anaerobic digestion of swine manure fibers pretreated with aqueous ammonia soaking. In: INTERNATIONAL SYMPOSIUM ON ENERGY FROM BIOMASS AND WASTE, 4., 2012, San Servolo, Venice. Proceedings... Venice: IWWG-International Waste Working Group, 2012. 1 CDROM.

KOSSEVA, M.; WEBB, C. Food industry wastes: assessment and recuperation of commodities. [Manchester]: Academic Press, 2013.

LANGTANGEN, H. P. Python scripting for computational science. 2nd ed. Berlin: Springer, 2006.

LAUWERS, J. et al. Structural identifiability analysis of the Anaerobic Digestion Model No. 1 using a local algebraic observability approach. IFAC-PapersOnLine, Kidlington, v. 48, n. 1, p. 470-475, 2015.

LEE, T.; LEE, Y. H. Modeling of thermophilic anaerobic digestion of municipal sludge waste using Anaerobic Digestion Model No. 1 (ADM1). In: INTERNATIONAL CONFERENCE ON ENVIRONMENT, ENERGY, ECOSYSTEMS AND DEVELOPMENT, 2013, Rhodes Island, Greece. Proceedings... Rhodes Island, Greece, 2013. p. 127-128.

LIU, G. et al. Research progress in anaerobic digestion of high moisture organic solid waste. Agriculture Engineering International: CIGR journal, Gainesville, v. 13, n. 9, 2007.

LOKSHA, I. V. et al. Systematic hierarchical algorithms for rotamers and proteins on an extended network. Journal of Computational Chemistry, Hoboken, v. 30, p. 999-1005, 2009.

LÜBKEN, M. et al. Modelling the energy balance of an anaerobic digester fed with cattle manure and renewable energy crops. Water Research, London, v. 41, p. 4085-4096, 2007.

LUTZ, M. Learning python. 5th ed. Beijing: O’Reilly Media, 2013.

LYRA, M. R. C. C.; ROLIM, M. M.; SILVA, J. A. A. Toposequence of soils fertigated with stillage: contribution towards the quality of ground water table. Revista Brasileira de Engenharia Agrícola Ambiental, 7: 525-32, 2003.

MALISKA, C. R. Transferência de calor e mecânica dos fluidos computacional: fundamentos e coordenadas generalizadas. Rio de Janeiro: LTC, 1995.

MCCRUM, I. T. The DARIES model: modeling co-digestion and multi-phase digester systems: a thesis. Universidade de Clarkson, 2012.

MEYERS, C. R.; SETHNA, J. P. Python for education: computational methods for nonlinear systems. Computing in Science \& Engineering, Piscataway, v. 9, n. 3, p. 75-79, 2007. 
MOHAMAD, A. A. Lattice Boltzmann method: fundamentals and engineering applications with computer codes. London: Springer-Verlag, 2011.

MU, S. J. et al. Anaerobic digestion model no. 1-based distributed parameter model of an anaerobic reactor: I. model development. Bioresource Technology, Amsterdam, v. 99, p. 3665-3675, 2008.

NAESSENS, W. et al. Critical review of membrane bioreactor models: part 2: hydrodynamic and integrated models. Bioresource Technology, Amsterdam, v. 122, p. 107-118, 2012.

OKIYAMA, D. C. G. Simulação numérica da hidrodinâmica de biorreator em leito fixo para tratamento de vinhaça. Tese de Mestrado. Faculdade de Zootecnia e Engenharia de Alimentos, Universidade de São Paulo, Pirassununga, 2013.

PERTUSI, D. A. et al. Efficient searching and annotation of metabolic networks using chemical similarity. Bioinformatics, Oxford, v. 31, n. 7, p. 1016-1024.

QUEEN, A. S. Simulador de reatores anaeróbios com base no ADM1. 2006. 98 f. Dissertação (Mestrado) - Escola Politécnica, Universidade de São Paulo, São Paulo, 2006.

RAYMOND, E. S. Why python? Linux Journal, Houston, 30 Apr. 2000. Disponível em: <https://www.linuxjournal.com/article/3882>. Acesso em: 10 jan. 2018.

ROSEN, C.; JEPPSSON, U. Aspects on ADM1 implementation within the BSM2 framework. Industrial Electrical Engineering and Automation, Lund, Sweden, p. 1-35, 2006.

SAGUY, I. S. Challenges and opportunities in food engineering: modeling, virtualization, open innovation and social responsibility. Journal of Food Engineering, London, v. 176, p. 2-8, 2016.

SAYAMA, H. Introduction to the modeling and analysis of complex systems. Geneseo: Open SUNY Textbooks, 2015.

SCHOEN, M. Numerical modelling of anaerobic digestion processes in agricultural biogas plants dissertation. 2009. 139 p. Dissertation (Doktor) - Fakultät Für Bauingenieurwissenschaften, Universität Innsbruck, Innsbruck, 2009.

SIEGRIST, H. et al. Mathematical model for meso- and thermophilic anaerobic digestion. Environmental Science \& Technology, Washington, v. 36, p. 1113-1123, 2002.

SILVA, F. et al. Modelling of anaerobic treatment of evaporator condensate (EC) form a sulphite pulp mill using the IWA anaerobic digestion model no.1 (ADM1). Chemical Engineering Journal, Amsterdam, v. 148, n. 2-3, p. 319- 326, 2009.

TARTAKOVSKY, B. et al. Anaerobic digestion model No. 1-based distributed parameter model of an anaerobic reactor: II. Model validation. Bioresource Technology, Amsterdam, v. 99, p. 3676-3684, 2008. 
TARUYANON, K.; TEJASEN, S. Modelling of two-stage anaerobic treating wastewater from a molasses-based ethanol distillery with the iwa anaerobic digestion model no.1. Engineering Journal, Thailand, v. 14, n. 1, p. 25-36, 2010.

VAVILIN, V. A. et al. Anaerobic digestion of solid material: Multidimensional modeling of continuous-flow reactor with nonuniform influent concentration distributions. Biotechnology and Bioengineering, v. 97, p. 354-366, 2007.

VEGA DE LILLE, M. et al. Modeling the two-stage anaerobic digestion of domestic wastewater with the development of a monitoring application. Brazilian Journal of Chemical Engineering, São Paulo, v. 33, n. 4, p. 801-815, 2016.

VERMA, S. Anaerobic digestion of biodegradable organics in municipal solid wastes. 2012. Master of Science Degree, Columbia University, 2012.

VON SPERLING, M. Basic principles of wastewater treatment. London: IWA Publishing, 2007. v. 2. (Biological Wastewater Treatment Series).

YU, L. et al. Mathematical modeling in anaerobic digestion (AD). Journal of Bioremediation and Biodegradation, Los Angeles, s4, art. 003, 2013.

WETT, B.; ELADAWY, A.; OGUREK, M. Description of nitrogen incorporation and release in ADM1. Water Science and Technology, Oxford, v. 54, n. 4, p. 67-76, 2006. 
9 APÊNDICE A - Matriz Petersen

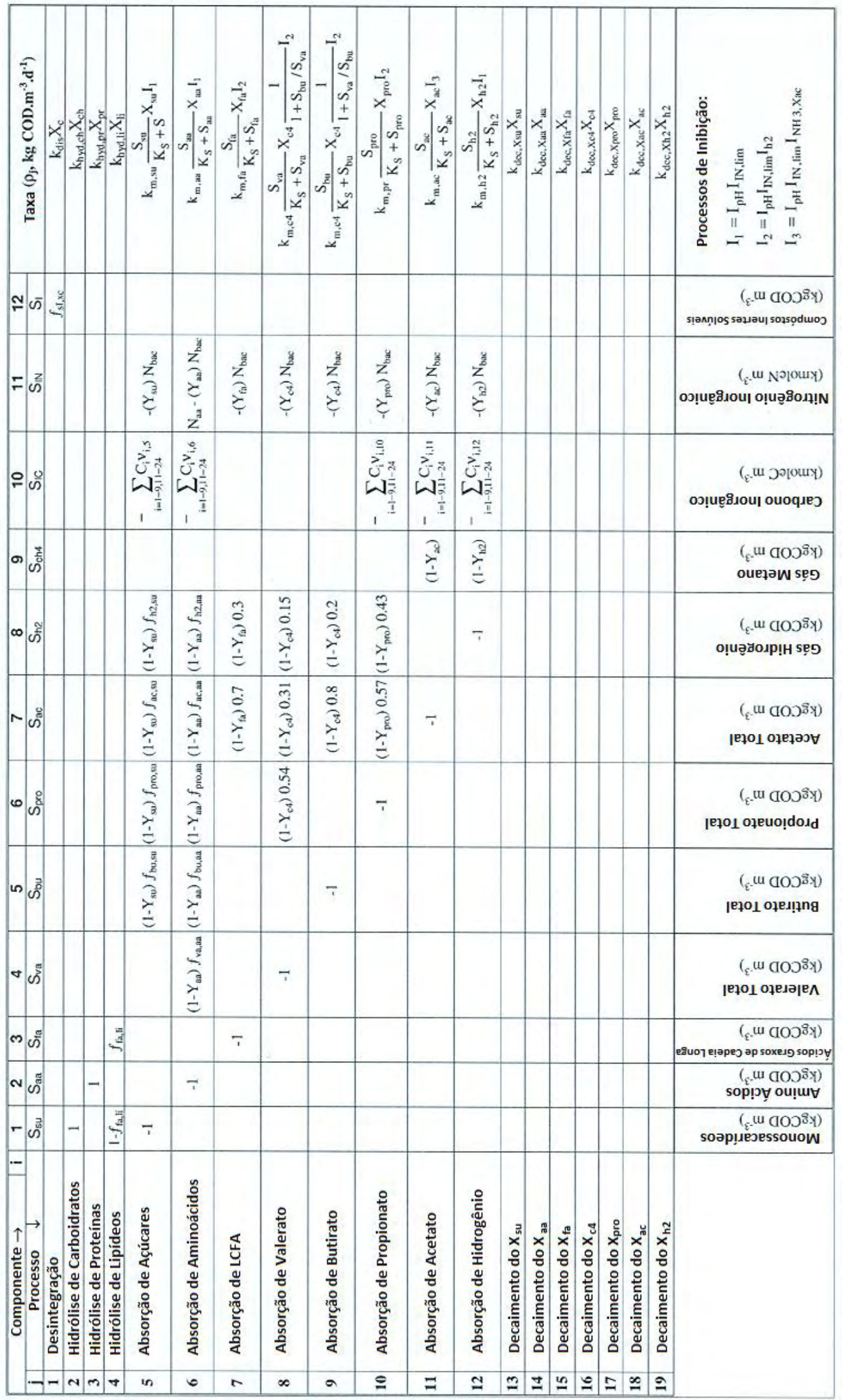




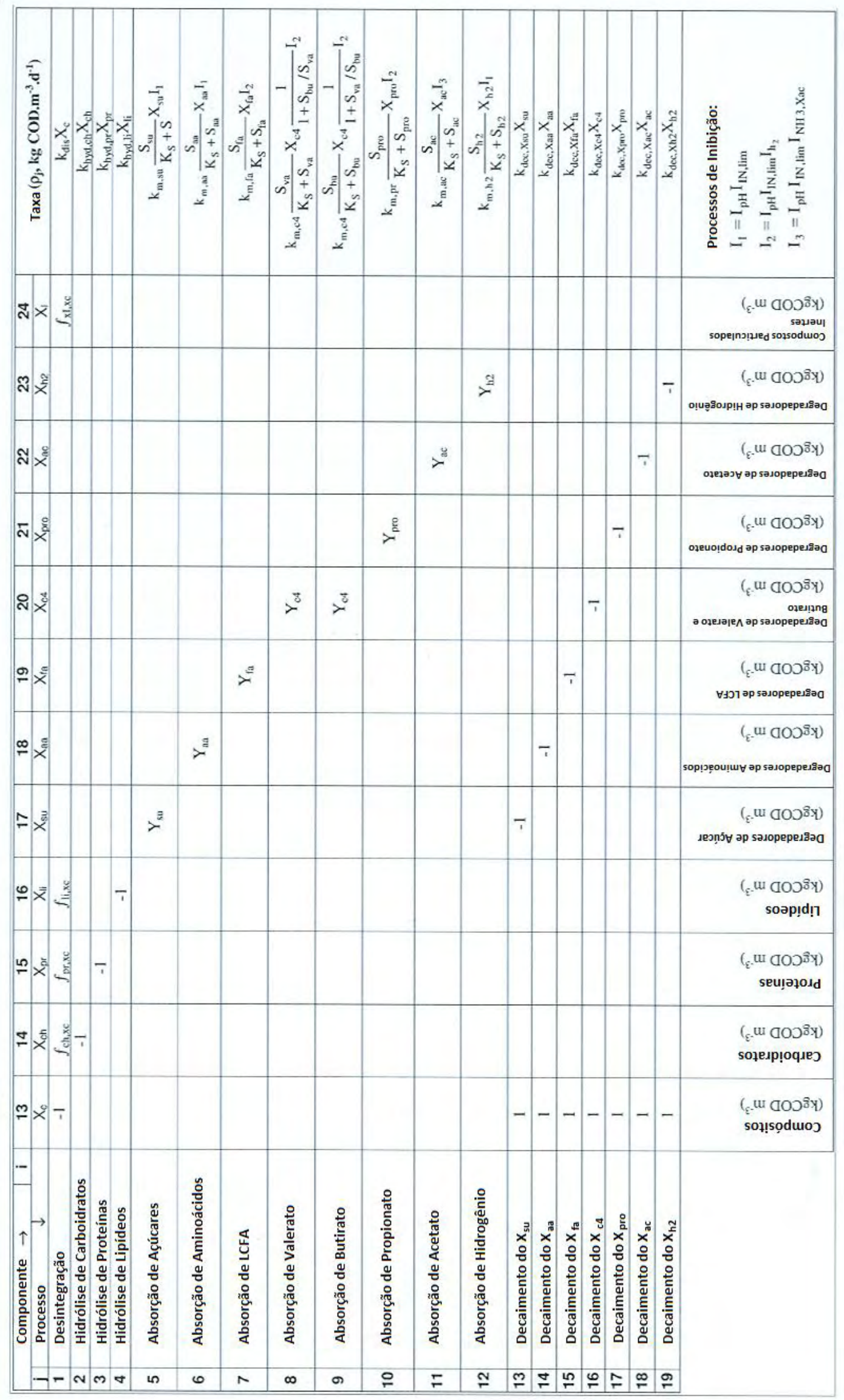

